DOT/FAA/AM-21/15

Aviation Safety

Office of Aerospace Medicine

Washington, DC 20591

\title{
Examining Minimum Information Requirements for Electronic Aeronautical Charts
}

\author{
Michelle Yeh ${ }^{1}$ \\ Joseph M. Jaworski \\ Cathy Swider ${ }^{1}$ \\ Stephanie Chase $^{3}$ \\ ${ }^{1}$ Federal Aviation Administration \\ Washington, DC 20591 \\ ${ }^{2}$ Cherokee Nation Support, Service, and Solutions \\ Oklahoma City, OK 73125 \\ ${ }^{3}$ Volpe National Transportation Systems Center \\ Cambridge, MA 02142
}

April 2021

Technical Report 


\section{NOTICE}

This document is disseminated under the sponsorship of the U.S. Department of Transportation in the interest of information exchange. The United States Government assumes no liability for the contents thereof.

This publication and all Office of Aerospace Medicine technical reports are available in full-text from the Civil Aerospace Medical Institute's publications Web site: (www.faa.gov/go/oamtechreports) 


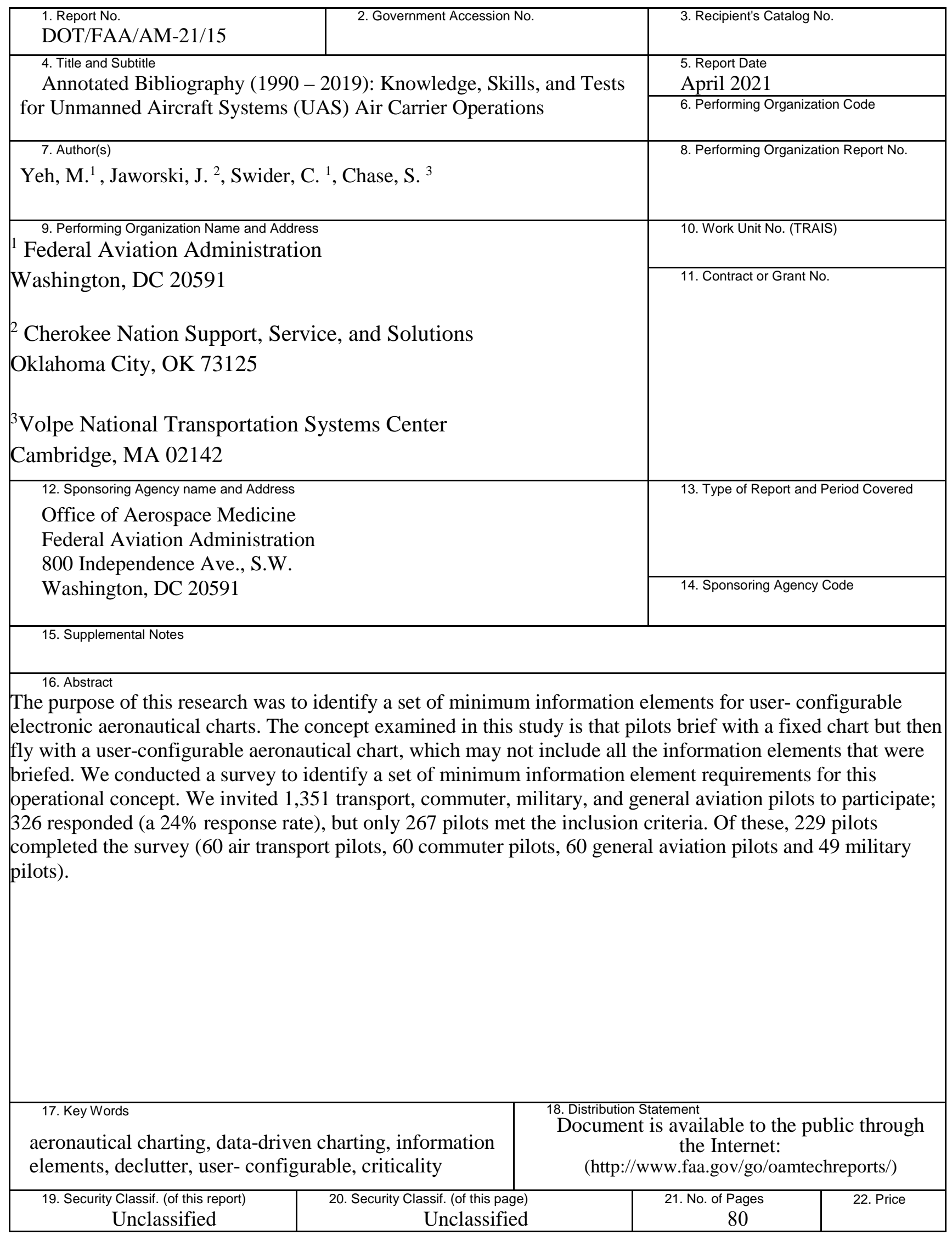




\section{Table of Contents}

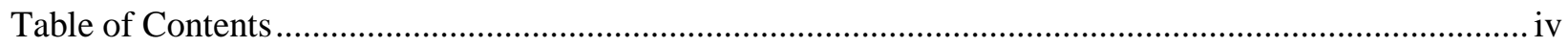

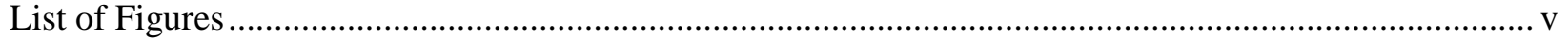

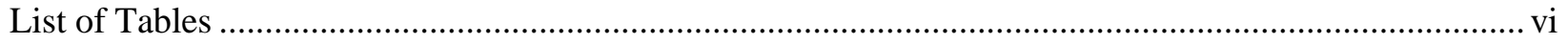

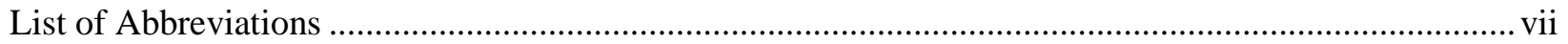

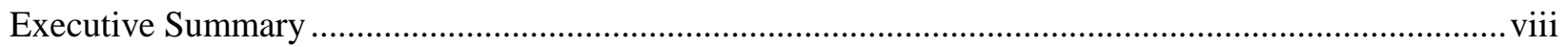

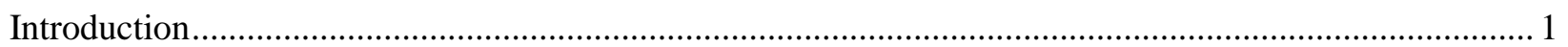

Previous Research on Aeronautical Chart Design....................................................................... 2

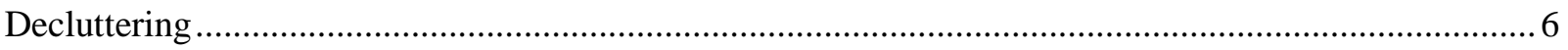

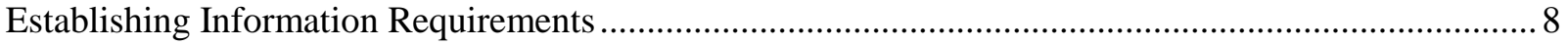

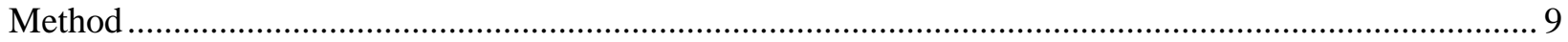

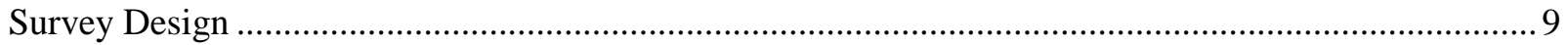

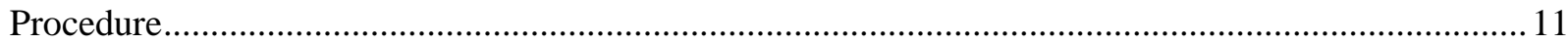

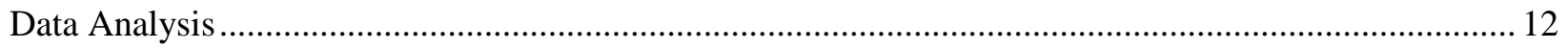

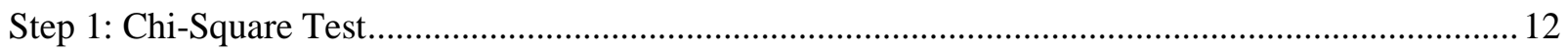

Step 2: Combined Subject Matter Expert Review + Analysis .......................................................... 13

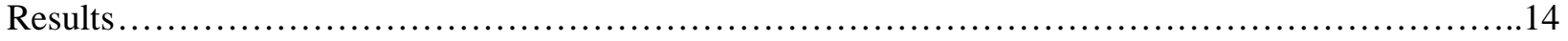

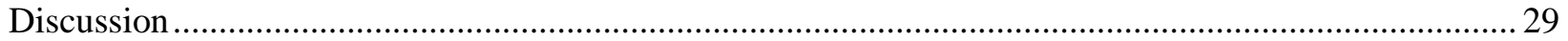

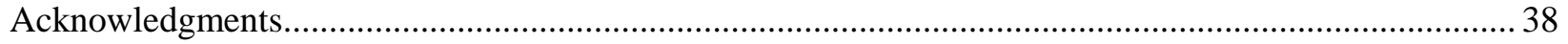

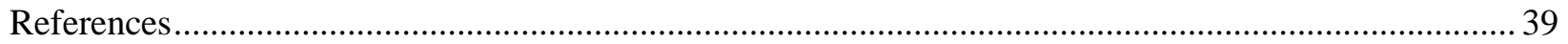

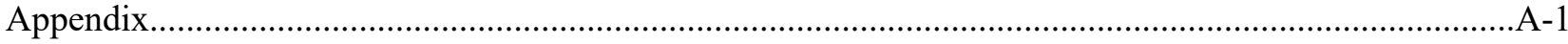




\section{List of Figures}

Figure 1 Example of a customizable chart integrated with other charting information............... 2

Figure 2 Comparison of Standard IAP Chart and Volpe/ATA Recommended IAP Chart

(Excerpted from Blomberg et al., 1995). ............................................................................... 4

Figure 3 Current FAA IAP Chart (Adapted from Blomberg et al., 1995). ................................. 5

Figure 4 Example of STAR survey with response options..................................................... 11

Figure 5 Mock-up example of Standard Terminal Arrival Route (STAR) Chart....................... 33

Figure 6 Mock-up example of Enroute Instrument Flight Rule (IFR) Chart............................. 34

Figure 7 Mock-up example of Standard Instrument Departure (SID) Chart........................... 35

Figure 8 Mock-up example of Instrument Approach Procedure (IAP) Chart........................... 36 


\section{List of Tables}

Table 1 Distribution of Participants by Pilot Type ............................................................... 11

Table 2 Chi-Square Analysis Categorization ....................................................................... 13

Table 3 Number of Information Elements by Importance Level ............................................. 15

Table 4 Survey Results for Instrument Approach Procedure (IAP) Charts ............................... 15

Table 5 Survey Results for Enroute Instrument Flight Rules (IFR) Charts ............................. 19

Table 6 Survey Results for Standard Instrument Departure (SID) Charts .............................. 22

Table 7 Survey Results for Standard Terminal Arrival Route (STAR) Charts........................... 26

Table 8 Comparison of Information Element Ratings: Results..............................................31 


\section{List of Abbreviations}

$\begin{array}{ll}\text { ARP } & \text { Aerospace Recommended Practice } \\ \text { ATA } & \text { Air Transport Association } \\ \text { FAA } & \text { Federal Aviation Administration } \\ \text { GPS } & \text { Global Positioning System } \\ \text { IAP } & \text { Instrument Approach Procedure } \\ \text { ICAO } & \text { International Civil Aviation Organization } \\ \text { IFR } & \text { Instrument Flight Rules } \\ \text { NOS } & \text { National Ocean Service } \\ \text { SID } & \text { Standard Instrument Departure } \\ \text { SME } & \text { Subject Matter Expert } \\ \text { STAR } & \text { Standard Terminal Arrival Route } \\ \text { US DOT } & \text { United States Department of Transportation } \\ \text { VFR } & \text { Visual Flight Rules }\end{array}$




\section{Executive Summary}

The purpose of this research was to identify a set of minimum information elements for userconfigurable electronic aeronautical charts. The concept examined in this study is that pilots brief with a fixed chart but then fly with a user-configurable aeronautical chart, which may not include all the information elements that were briefed. We conducted a survey to identify a set of minimum information element requirements for this operational concept. We invited 1,351 transport, commuter, military, and general aviation pilots to participate; 326 responded (a 24\% response rate), but only 267 pilots met the inclusion criteria. Of these, 229 pilots completed the survey (60 air transport pilots, 60 commuter pilots, 60 general aviation pilots and 49 military pilots).

The survey was comprised of lists of information elements shown on four types of aeronautical charts: 1) Instrument Approach Procedure (IAP), 2) Enroute Instrument Flight Rules (IFR), 3) Standard Terminal Arrival Route (STAR), and 4) Standard Instrument Departure (SID). There were a total of 427 information elements across charts, so to prevent survey fatigue, we divided the information elements into two surveys. The first survey included information elements on IAP/Enroute IFR charts (221 information elements), and the second survey included information elements from SID/STAR charts (206 information elements).

For each survey, participants were instructed to rate the importance of information elements for a new charting concept, which uses customizable electronic charts that are interactive and customized to display only information elements needed to execute the procedure. Participants were instructed that for this charting concept, they would first brief the procedure using a fixed chart showing all the information elements, but then fly with a customized electronic chart, which may or may not include all the information elements that were briefed. Additionally, the instructions emphasized that the customizable electronic chart would show only the information relevant to the procedure being flown.

Participants were given a list of information elements and asked to rate the importance of each information element when using a customizable electronic chart while executing the procedure and not on the frequency of use. Ratings were made along a scale with four options for level of importance, with an additional option for participants who did not know the information element:

- $\quad 1$ = Required to be displayed continuously for the safe and successful execution of the instrument flight procedure;

- $\quad 2$ = Displayed initially, but can be removed and recalled for reference, as needed;

- 3 = Not displayed initially, but can be displayed manually for reference, as needed;

- 4 = Not required to execute the procedure;

- Don’t know/Unsure. 
We analyzed the data using one-way chi-square tests and consulted with subject matter experts to identify a criticality level for each information element. Based on this analysis, we were able to categorize $85 \%$ of the information elements across all four chart types. (The ratings for each information element are shown in Tables $4-7$ by chart type). We then developed prototype charts to visualize what the concept might look like. 


\section{Introduction}

Aeronautical charting has evolved with changes in display mediums, display technology, expanded use of the Global Positioning System (GPS), and increased information processing capabilities. The term “aeronautical chart” refers to a map used to provide air navigation information for pilots, much like a road map for drivers. The aeronautical chart shows information such as navigation routes or airways, navigation aids, airspace boundaries, topographic features, and airports. Pilots are required to fly with aeronautical charts; they typically use the chart to brief the procedure as part of flight planning, and may then put the chart away until they need to execute the procedure.

Much like maps that have evolved from paper to electronic media, the aeronautical chart has evolved from paper to electronic format. There are three types of electronic charts: raster, vector, and electronic data-driven. Raster charts are electronic images of paper charts, so a raster chart will show the exact same information as its paper counterpart. Own-aircraft position may be displayed on aeronautical raster charts only if the chart is georeferenced. Vector charts look similar to raster charts, but the information elements are mathematically encoded in a database. This encoding allows functionality such as resizing of symbols and text when the pilot zooms in (or out) or when information is filtered. A vector chart is computer generated, so it may not look the same as a paper chart. Finally, electronic data-driven charts may be user-configurable so that

information elements can be added or removed via a manual or automatic decluttering capability. This third type of charting is the focus of this research.

With each evolution, the usability of the aeronautical chart needs to be considered, as many of the human factors considerations have remained the same regardless of display medium (e.g., display clutter, readability/legibility, symbology, to name a few). As electronic charts become integrated into flight decks, the design of the electronic chart may diverge depending on the manufacturer's design philosophy. For example, the electronic chart symbology can become more specific to the task at hand, using manual or automatic decluttering methodologies that are customizable by the pilot, and the chart can be integrated with other map information. An example of what this type of chart might look like is shown in Figure 1. 


\section{Figure 1}

Example of a customizable chart integrated with other charting information.

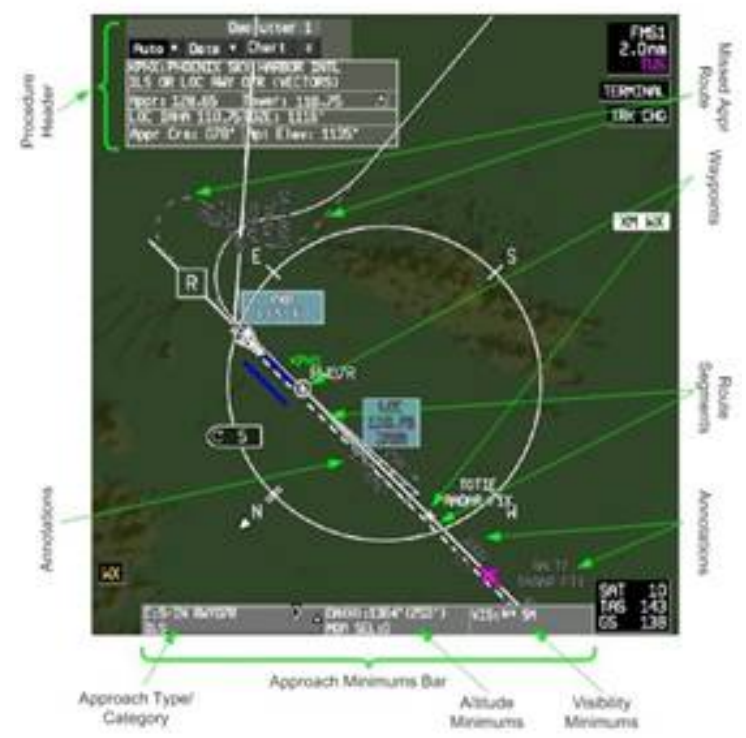

The purpose of this paper is to discuss the human factors considerations applicable to the design of electronic aeronautical charts. The Federal Aviation Administration (FAA) was interested in understanding whether a minimum set of information elements could be defined for these customizable electronic charts. We start by reviewing past research on the design of aeronautical charts and then highlight some of the recurring human factors issues addressed in each evolution.

\section{Previous Research on Aeronautical Chart Design}

Research in aeronautical chart design has focused primarily on methods for improving visual search. In the 1990s, researchers at the United States Department of Transportation (US DOT) Volpe National Transportation Systems Center ("Volpe Center”) conducted a series of studies to improve the design layout of Instrument Approach Procedure (IAP) charts. ${ }^{1}$

The research studies examined different formatting techniques for presenting heading information (Multer et al., 1991), different layouts for presenting frequency information (Multer

\footnotetext{
${ }^{1}$ An IAP chart shows pilots the information needed to descend and land when they are flying using the instruments on the flight deck. Note that this in contrast to flying under Visual Flight Rules (VFR) in which a pilot operates an aircraft in clear weather conditions.
} 
et al., 1991), use of text or graphical icons for finding missed approach instructions (Osborne \& Huntley, 1992), and different layouts for planning and executing an approach or missed approach (Blomberg et al.,1995; Osborne et al., 1995).

Multer et al. (1991) examined ways to facilitate visual search on IAPs by evaluating different formatting for heading information (such as font size, bolding, and highlighting), as well as different layouts for frequency information. Airline pilots were asked to identify the heading on a number of fictional IAPs as quickly as possible. In the first study, the text on charts varied in terms of font size and highlighting method (plain type, bold type, boxed, or reverse video). The results showed an interaction between font size and highlighting; when text was bolded or boxed, response time decreased as font size increased, but when text was presented using reverse video, there was no impact of font size.

In the second study, four different spatial layouts were used for showing frequency information - two were based on existing US chart provider designs, the third was a boxed layout used in Canada, and the fourth was a new two-column layout. The results showed that the use of a boxed format and the two column layout, which used space to organize text placement, facilitated search relative to the one-column format used by US chart providers.

Osborne and Huntley (1992) examined whether the use of text or graphical icons could help pilots retrieve missed approach instructions. Pilots were asked to read information from National Ocean Service (NOS) IAP charts shown at one of three information density levels: low, medium, and high. Pilot comprehension speed, measured by the number of glances needed for pilots to read and verbally repeat the instructions, were recorded. Overall, pilots identified information more quickly and accurately when there was a low level of information density, and the slowest with a high level of information presented. Pilots expressed preference for icon information rather than text, but thought that some icons needed clarification.

The US DOT Volpe Center also collaborated with the Air Transport Association (ATA) Chart and Data Display Working Group to provide guidance on pilot information requirements. As part of this collaboration, Blomberg et al. (1995) compared a standard IAP chart design with two new prototype charts, as shown in Figure 2. Pilots completed a series of tasks used to obtain feedback about the charts. First, they were asked to fly 10 simulated approaches using one of the 
prototype charts and the standard chart and then debriefed about their experiences. Second, pilots were shown a different prototype chart and asked for their opinions, but they did not fly with this chart. Third, pilots ranked the three charts on their perceived usefulness for executing an approach. Finally, pilots were asked to assume that s/he was responsible for making a purchase decision to be used by all pilots working for his/her airline. Findings showed that despite pilots' concerns with accepting a new chart design, pilots had a higher preference for the Volpe/ATA prototype chart.

\section{Figure 2}

Comparison of Standard IAP Chart and Volpe/ATA Recommended IAP Chart (Excerpted from

Blomberg et al., 1995).
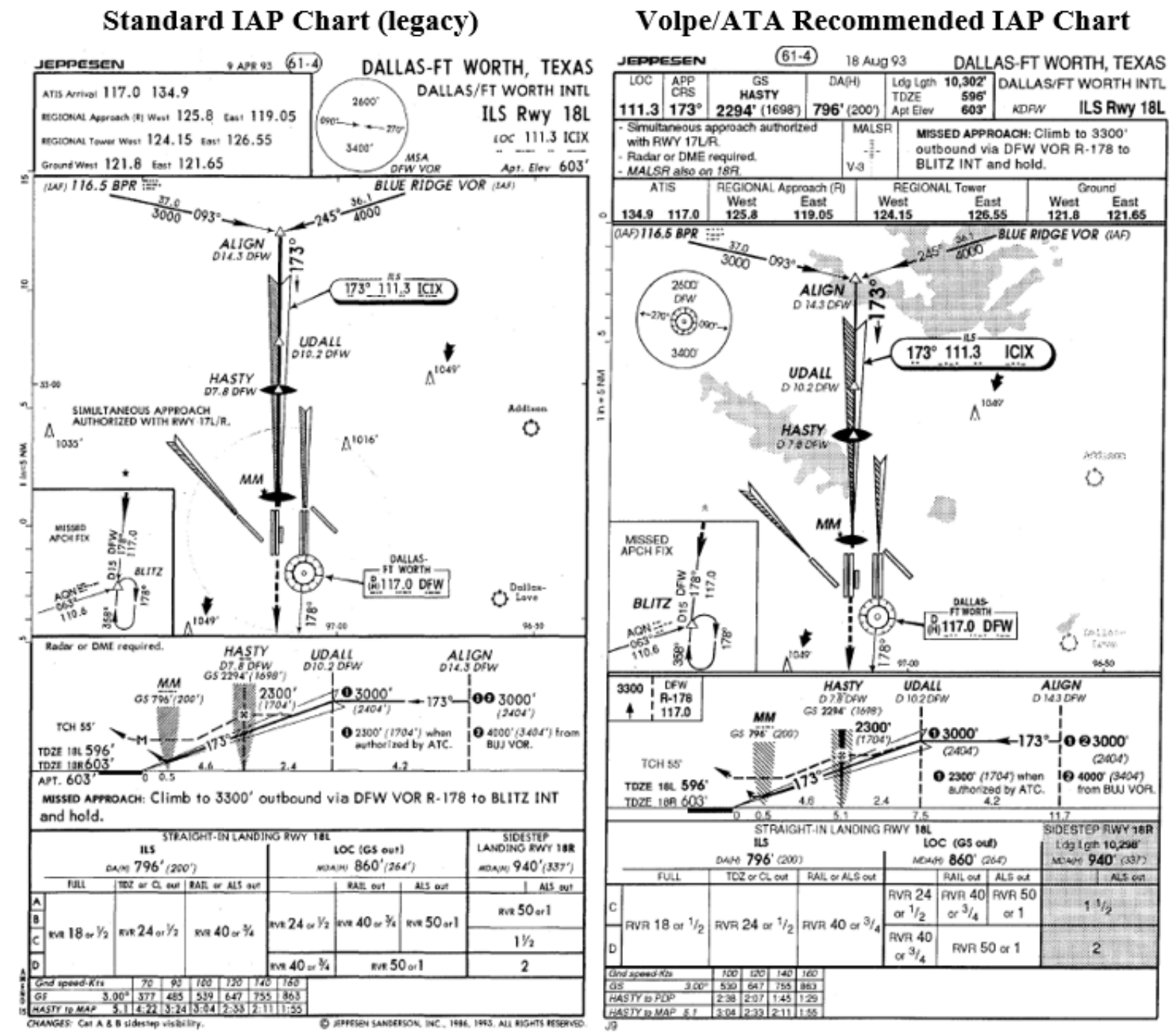
The chart evaluated by Blomberg et al. (1995) was refined during field testing and subject matter expert reviews, as shown in Figure 3. The addition of the briefing strip at the top of the chart was intended to promote briefing as a critical component of flying an approach, and to present the required information in a logical order in one place. A boxed layout was used to show heading and frequency information and graphical icons were added to depict missed approach information.

Osborne et al. (1995) evaluated the usability of this new prototype IAP chart by asking pilots to fly approaches in a simulator. Pilots used a standard IAP chart or the Volpe IAP prototype. During the flight, pilots were asked questions that required him/her to search for information located on the chart. Pilots found information much faster on the Volpe prototype chart than on the standard IAP chart, particularly when that information was located in the briefing strip. Additionally, pilots’ accuracy did not differ between questions using the standard and prototype charts. The recommended IAP chart was adopted by Jeppesen and also had a marked influence on design of the current FAA IAP chart.

\section{Figure 3}

Current FAA IAP Chart (Adapted from Blomberg et al., 1995).

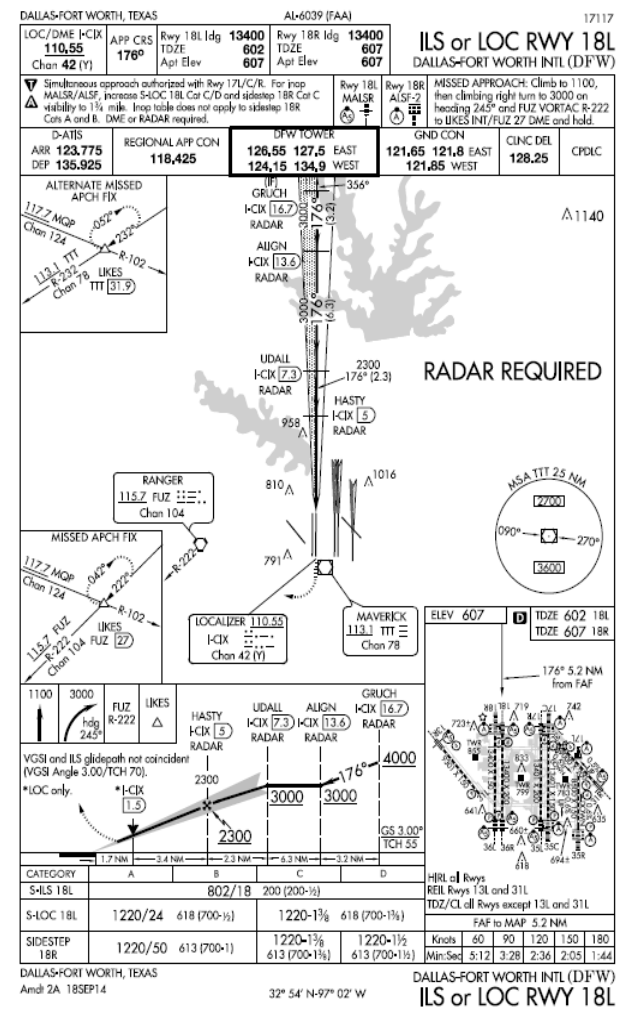




\section{Decluttering}

Figure 3 also shows the level of detail and high information density on aeronautical charts. This may lead to the perception of “clutter," which has the consequences of slowing visual search, increasing memory load, and negatively impacting position awareness (Moacdieh \& Sarter, 2015). The costs of clutter are seen most heavily in visual search (Teichner \& Mocharnuk, 1979), but may also be reflected in information readout, when an information element is found but cannot be discriminated because other information elements are in close spatial proximity. Visual search often occurs serially, in which each item is examined until the "target" information element is found. The more information that is on the search space, the longer it will take until the "target" is found. General techniques to filter attention may facilitate information search as a result of the preattentive nature of information processing (e.g., by color or intensity). One can discriminate color and intensity relatively early and automatically, so unique colors or intensity differences can produce "automatic" detection, sometimes described as a “pop-out” effect (Treisman, 1988; Treisman \& Gelade, 1980; Yantis, 1993). That is, different color and intensity makes it easier to locate and focus attention on information elements, and reduces distraction created by other information elements.

Another series of research studies has focused on reducing clutter on aeronautical charts by removing information elements that may not be critical or relevant for the current task. In particular, groups of information elements on the display could be “decluttered” (i.e., hidden) so that they do not interfere with task performance. Several pilot surveys have been conducted to develop concepts for organizing and layering information, so that information elements can be added or removed. Hansman and Mykityshyn (1995a) addressed this topic for instrument approach charts, Yeh and Chandra (2003) for surface moving maps, and Schvaneveldt et al. (2001) for flying in general. Collectively, the results showed that "critical” information elements differed depending on the phase of flight.

Hofer et al. (1993) applied decluttering in high information density paper approach charts, and noted that the ability to retrieve "decluttered" information must be considered in the chart design. Hansman and Mykityshyn (1995b) reported that pilots were interested in the ability to declutter information but were concerned about the ability to retrieve the suppressed 
information when needed. Additionally, if information is not visible, the pilot may not remember it is available and fail to consider the information when it is relevant.

Butchibabu and Hansman (2012) conducted a review of several aeronautical charts to understand whether each information element contributed to operational issues. The results indicated that charts that depicted more flight paths resulted in more operational issues than charts with fewer flight paths, and on those "problematic" charts, each flight path consisted of more information elements per path than on charts with fewer issues. Butchibabu and Hansman then conducted a study to examine whether clutter could be mitigated by reducing the number of flight paths shown on one aeronautical chart, and distributing the information across multiple pages. They asked pilots to find information on both decluttered electronic aeronautical charts as well as the standard chart. The results showed that pilots found the information significantly faster using the decluttered charts, but the information being retrieved was always shown on the electronic chart (i.e., pilots were never asked to find information about a flight path that was on a different decluttered chart than the one displayed).

Stewart et al. (2016) also attempted to simplify the depiction of instrument approach charts by decluttering irrelevant information. Their study focused on the presentation of approach minimums; specifically, the minimum altitude at which the pilot must see the runway or execute a missed approach, and the minimum visibility, which is the lowest visibility authorized for the approach. An approach procedure on an instrument approach chart needs to accommodate multiple users and aircraft types, but this information density increases the likelihood that the pilot could select an incorrect minimum.

Stewart et al. (2016) created prototype charts that showed only approach minima relevant to the specific pilot and specific aircraft type to prevent pilots from selecting incorrect information. Additionally, they added data labels and color coding, which helped clarify the data type to increase the likelihood that pilots would select the correct information. Pilots were able to identify the information they needed on a decluttered prototype chart and standard instrument approach charts faster and made fewer errors with the decluttered chart. However, it is not clear if the improved search time was solely attributable to decluttering or if the other changes also contributed to faster search times. 


\section{Establishing Information Requirements}

Collectively, the results suggest that decluttering facilitates visual search and reduces errors when combined with other formatting techniques (e.g., by bolding or highlighting). New concepts are being proposed that incorporate aircraft state or information on flight planning to create data-driven charting systems and to integrate aeronautical charting information with a moving map on a single display. We were interested in identifying a minimum set of information elements for such a concept by examining the criticality of the information elements shown on the aeronautical charts.

We started by reviewing SAE Aerospace Recommended Practice (ARP) 5621, Electronic Display of Aeronautical Information (SAE International, 2011), which provides a categorization of information elements based on subject matter expert opinion for electronic charts intended to be used as a replacement for paper charts. The SAE Committee considered nine chart types; for each chart type, the Committee identified a set of information elements shown on the charts and discussed the criticality of each information element for presentation on a fixed chart for briefing or a moving map format for flying the procedure. The ratings were based on the following criteria:

- Level 1: information elements that cannot be removed

- Level 2: information elements that should be shown initially but could be removed by pilot action

- Level 3: information elements that do not need to be presented initially and can be manually selected (or deselected).

SAE ARP 5621 provided only a starting point for this research, as the criticality ratings were not validated when that document was published. In 2014, Pepitone et al. conducted a preliminary validation of a subset of the information in SAE ARP 5621. Twenty Honeywell corporate pilots participated in a card-sorting task in which they rated the criticality of the information elements for flying an Instrument Flight Rules (IFR) procedure using three rating levels, similar to the ones used in SAE ARP 5621. Pepitone et al.'s results provided some validation of the SAE ratings; there were some differences, but the study was limited in that the 
data reflected the opinions of corporate pilots from one company only, and no statistical analyses were reported.

Therefore, the purpose of this study was to further examine the criticality ratings provided in SAE ARP 5621 to try to identify minimum information requirements for electronic data-driven charts. This study focused on four different chart types- Instrument Approach Procedure (IAP), Enroute IFR, Standard Terminal Arrival Route (STAR), and Standard Instrument Departure (SID). We recruited pilots to participate from four different types of operations - air transport, commuter/business jet, military, and general aviation.

\section{Method}

We developed a survey and distributed it to air transport, commuter, military, and general aviation pilots. The inclusion criteria for participation was that pilots needed to have flown IFR in the previous 6 months and use FAA/US Government (military) or Jeppesen charts. A total of 1,351 pilots were invited to participate; 326 responded (24\% response rate), but only 267 met the inclusion criteria. Of these, 229 pilots completed the survey (218 male, 10 female, and 1 did not self-identify): 60 air transport pilots, 60 commuter, 60 general aviation, and 49 military pilots. The average age was 45 years $(\operatorname{Min}=19 ; \operatorname{Max}=73$ ). For chart usage, 128 pilots indicated that they primarily used Jeppesen charts, and 101 pilots indicated they primarily used FAA or US Government charts.

\section{Survey Design}

We created a list of information elements shown on four types of charts (IAP, Enroute IFR, STAR, and SID). There were a total of 427 information elements, so to prevent survey fatigue, we divided the information elements into two surveys: the first survey included information elements on IAP/Enroute IFR charts (221 information elements), and the second survey included information elements from SID/STAR charts (206 information elements).

When completing a survey, participants were instructed to rate the importance of information elements for a new charting concept, which uses customizable electronic charts that are interactive and customized to display only information elements needed to execute the procedure. Participants were instructed that for this charting concept they would first brief the procedure using a fixed chart showing all the information elements, but then fly with an 
electronic chart, which may or may not include all the information elements that were briefed. Additionally, the instructions emphasized that the customizable electronic chart would show only the information relevant to the procedure being flown.

Participants were given a list of information elements and asked to rate each information element individually with respect to how the information element would be used operationally when executing the procedure. Pilots were not asked to rate the information element based on the frequency of use. Ratings were made along a scale with four levels of importance options with an additional option level for participants who did not know the information element.

- 1 = Required to be displayed continuously for the safe and successful execution of the instrument flight procedure.

- 2 = Displayed initially, but can be removed and recalled for reference, as needed.

- 3 = Not displayed initially, but can be displayed manually for reference, as needed.

- 4 = Not required to execute the procedure.

- Don’t know/Unsure

To help with the task, participants were shown charts that depicted the information elements being rated (see Figure 4). Because the symbology for each chart type may differ depending on the chart provider, participants were shown charts designed by the chart provider the participant used most (i.e., FAA/US Government charts or Jeppesen charts). However, not all information elements being rated were depicted on the charts; these information elements were denoted by an asterisk in the survey. 


\section{Figure 4}

Example of STAR survey with response options.

\section{Standard Terminal Arrival Routes}

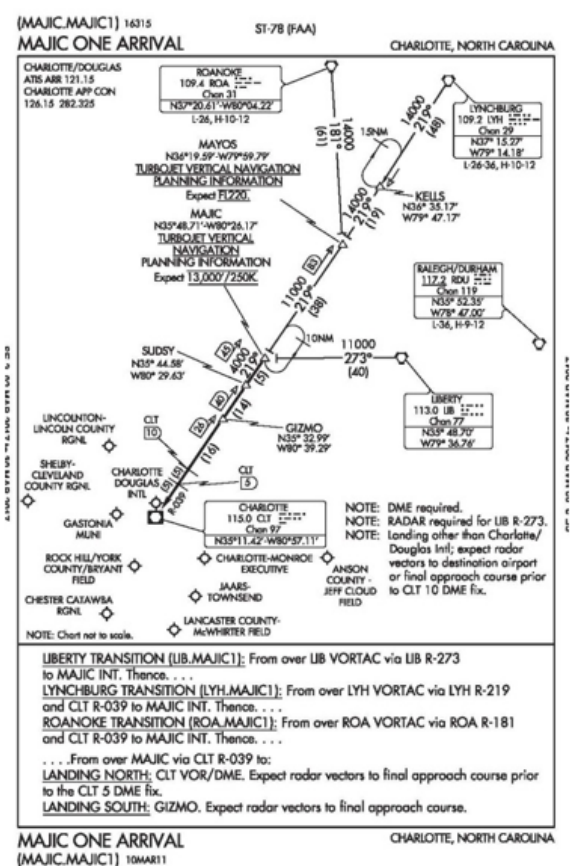

STARs1. Focusing on the chart as an example of STARs Charts, use the scale below to rate the importance of display elements when using a customizable electronic chart to execute a procedure.

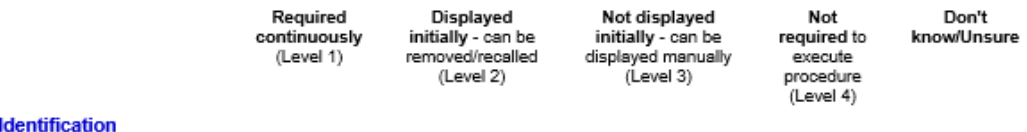

Chart Index Number/Page

Number

City/Location Name

Airport Name

Airport ICAO Identifier*

Procedure Name (e.g.

Canoge Eight)

Procedure Identifier (e.

CNOG8.VNY

Changes*

0

.

0

o 0

$\begin{array}{ll}0 & 0 \\ 0 & 0\end{array}$

$\begin{array}{llll}0 & 0 \\ 0 & 0 & 0\end{array}$

o 0

* Denotes display elements that may not be represented on the example chert.

Click the box below to review importance definitions.

a Review definitions

Note. Left panel shows the example aeronautical chart. Right panel shows the information element names to be rated.

\section{Procedure}

Participants were randomly assigned to either the IAP/Enroute IFR survey or the SID/STAR survey; 114 pilots completed the IAP/Enroute IFR survey, and 115 completed the SID/STAR survey. The number of participants by pilot type are shown in Table 1. The median time to complete the IAP/Enroute IFR survey was 38.5 minutes; the median time to complete the SID/STAR was 28.9 minutes. Pilots who completed a survey were compensated for their time.

\section{Table 1}

Distribution of Participants by Pilot Type

\begin{tabular}{lcc}
\hline \multicolumn{1}{c}{ Pilot Type } & IAP/IFR Participants & SID/STAR Participants \\
\hline Air Transport & 30 & 30 \\
Commercial & 30 & 30 \\
General Aviation & 30 & 30 \\
Military & 24 & 25 \\
\hline
\end{tabular}




\section{Data Analysis}

\section{Step 1: Chi-Square Test}

We conducted a series of one-way chi-squares for each information element to determine pilot ratings of importance $(p<0.05)$. The following questions were used to guide the analysis.

1. Did the majority of pilots feel that the information element should be displayed on the chart to successfully execute the procedure?

This was examined using a chi-square test to compare the total number of pilots who chose responses 1 , 2, or 3 to the number of pilots who chose response 4 (not required to execute the procedure).

- If the number of pilots who responded 1,2 , or 3 was significantly greater than the number of pilots who responded $4(p<0.05)$, then we asked question 2 .

- If the number of pilots who responded 4 was significantly greater than the number of pilots who responded 1,2 , or $3(p<0.05)$, then we concluded that pilots did not feel they needed the information element to execute the procedure. No further comparisons were conducted.

2. Did the majority of pilots feel that the information element should be displayed at all times?

This was examined using two chi-square tests: (1) we compared the number of pilots who chose response 1 to the number of pilots who chose response 2, and (2) we compared the number of pilots who chose response 1 to the number of pilots who chose response 3 .

- If the number of pilots who chose response 1 was significantly greater than the number of pilots who chose responses 2 or 3 ( $p<0.05$ ), then the majority of pilots felt that the information element should be displayed at all times.

- If the number of pilots who chose response 2 was significantly greater than the number of pilots who chose response $1(p<0.05)$, then the majority of pilots felt that the information should be displayed initially.

- If the number of pilots who chose response 3 was significantly greater than the number of pilots who chose response $1(p<0.05)$, then the majority of pilots felt that the information element did not need to be displayed initially.

- If the analysis was not significant ( $p>0.05$ ), then we concluded that there was no preference for whether it needed to be displayed at all times (not yet determined).

3. For pilots who felt information elements were not required to be displayed at all times, did the majority of pilots feel that the information element should be displayed initially? This was examined using chi-square tests comparing the number of pilots who chose response 2 to the number of pilots who chose response 3. 
- If the number of pilots who chose response 2 was significantly greater than the number of pilots who chose $3(p<0.05)$, then we concluded that the information element should be displayed initially.

- If the number of pilots who responded 3 was significantly greater than the number of pilots who responded $2(p<0.05)$, then we concluded that the information element did not need to be displayed initially.

- If the analysis was not significant ( $p>0.05$ ), then we concluded that the information element should be displayed part of the time, but that there was no preference for whether it needed to be displayed initially (not yet determined).

For each of these analyses we performed the Bonferroni correction on a subset of the information elements and found that the significance of the results were unchanged. We do not report those numbers here.

From this analysis, we were able to categorize 237 of the 427 information elements, as shown in Table 2 below.

Table 2

Chi-Square Analysis Categorization

\begin{tabular}{lc}
\hline Information Element Importance Level & $\begin{array}{l}\text { Number of Information } \\
\text { Elements }\end{array}$ \\
\hline Level 1 (Displayed at all times) & 135 \\
Level 2 (Displayed initially; can be toggled off/on) & 87 \\
Level 3 (Not displayed initially, can be toggled on/off) & 15 \\
Level 4 (Not displayed at all) & 0 \\
Not Yet Determined & 190 \\
\hline
\end{tabular}

\section{Step 2: Combined Subject Matter Expert Review + Analysis}

For the remaining 190 information elements that could not be categorized in Step 1 (Not Yet Determined), we requested feedback from two subject matter experts (SMEs), conducted an additional chi-square analysis, and looked for agreement in the two data sources.

SME Review: We recruited two SMEs to review the survey ratings: one SME primarily used FAA charts and the other primarily used Jeppesen charts. Both pilot SMEs provided their feedback voluntarily without monetary compensation. 
We provided each SME individually with the preliminary survey results from Step 1 and asked them to review the categorization of the information elements to determine if the survey ratings were consistent with their operational use of the information elements. We also asked the SMEs to review the information elements that did not clearly fall into one category (Not Yet Determined) and provide a category recommendation based on the information element's relationship with other information elements. For example, some information elements may need to be displayed together (e.g., airport identifier and airport symbol), but were rated as different levels of importance.

One SME focused his review on the information elements $(n=190)$ that could not be placed in a category through the chi-square analysis conducted in Step 1. That SME provided his recommendation between those levels based on the perceived relationship of the specific information element with other information elements at the same level. The second SME was presented with the same task, but rather than focus on the information elements that were classified as "Not Yet Determined," he also reviewed information elements that had already been categorized based on significance.

Analysis: We conducted a second chi-square analysis focused on the two importance levels with the highest ratings and reduced the level of significance from $p<0.05$ to $p<0.10$ (marginal significance) to see if we could classify the remaining items in conjunction with the feedback from the SMEs.

We utilized a flow chart to collectively look at SME feedback, the second chi-square analysis, and pilot categorization to determine a majority agreement across the sources. Through this method, we were able to classify an additional 127 of the 190 previously undetermined information elements.

\section{Results}

A total of 364 information elements were assigned an importance level (see Table 3), leaving 63 information elements not yet determined. These information elements that were classified as "not yet determined" did not have a majority of agreement among the sources of data. The chisquare analysis results are shown in Appendix A. 
Table 3

Number of Information Elements by Importance Level

\begin{tabular}{lc}
\hline Information Element Importance Level & Number of Information Elements \\
\hline Level 1 (Displayed at all times) & 173 \\
Level 2 (Displayed initially; can be toggled off/on) & 156 \\
Level 3 (Not displayed initially, can be toggled on/off) & 34 \\
Level 4 (Not displayed at all) & 1 \\
Not Yet Determined & 63 \\
\hline
\end{tabular}

The results of our survey are presented in Tables $4-7$. Table 4 shows the ratings for IAP charts, Table 5 presents Enroute IFR chart ratings, Table 6 shows the ratings for SID charts, and Table 7 displays the ratings for STAR charts. We show our results in comparison to SAE ARP 5621 (SAE International, 2011) and Pepitone et al. (2014) as a reference to the previous efforts aimed at identifying information element importance. We discuss these comparisons in detail further below. The information elements in the SAE ARP 5621 that are marked with an asterisk indicate the information element would be considered a Level 1 if the information element appeared on the pilot's planned route.

\section{Table 4}

Survey Results for Instrument Approach Procedure (IAP) Charts

\begin{tabular}{|c|l|l|c|c|c|}
\hline $\begin{array}{c}\text { Chart } \\
\text { Type }\end{array}$ & Information Category & Information Element & $\begin{array}{c}\text { SAE ARP } \\
\mathbf{5 6 2 1} \\
\mathbf{( 2 0 1 1 )}\end{array}$ & $\begin{array}{c}\text { Pepitone } \\
\text { et al. } \\
\mathbf{( 2 0 1 4 )}\end{array}$ & $\begin{array}{c}\text { Current } \\
\text { Survey }\end{array}$ \\
\hline IAP & Communications & Approach Frequency & 2 & 1 & $\begin{array}{c}\text { Not Yet } \\
\text { Determined }\end{array}$ \\
\hline IAP & Communications & ATIS Arrival Frequency & 3 & - & 2 \\
\hline IAP & Communications & ATIS Departure Frequency & 3 & - & 2 \\
\hline IAP & Communications & Clearance Frequency & 3 & - & 2 \\
\hline IAP & Communications & Departure Control Frequency & 3 & - & 2 \\
\hline IAP & Communications & Ground Frequency & 2 & 2 & 2 \\
\hline IAP & Communications & Helicopter Frequency & 1 & 3,4 & 3 \\
\hline IAP & Communications & Tower Frequency & 3 & 1 & 1 \\
\hline IAP & Geography & Contour Interval Legend & 3 & - & $\begin{array}{c}\text { Not Yet } \\
\text { Determined }\end{array}$ \\
\hline IAP & Geography & Cultural Features & 2 & - \\
\hline IAP & Geography & $\begin{array}{l}\text { Highest Reference Point (within neat } \\
\text { lines }\end{array}$ & 2 & 2 & $\begin{array}{c}\text { Not Yet } \\
\text { Determined }\end{array}$ \\
\hline
\end{tabular}




\begin{tabular}{|c|c|c|c|c|c|}
\hline $\begin{array}{l}\text { Chart } \\
\text { Type }\end{array}$ & Information Category & Information Element & $\begin{array}{c}\text { SAE ARP } \\
5621 \\
(2011) \\
\end{array}$ & $\begin{array}{c}\text { Pepitone } \\
\text { et al. } \\
(2014) \\
\end{array}$ & $\begin{array}{l}\text { Current } \\
\text { Survey }\end{array}$ \\
\hline IAP & Geography & $\begin{array}{l}\text { International Boundaries (higher } \\
\text { criticality where appropriate) }\end{array}$ & 2 & 2 & 2 \\
\hline IAP & Geography & Magnetic Variations & 3 & - & 3 \\
\hline IAP & Geography & $\begin{array}{l}\text { Neat Lines (i.e., the lines which } \\
\text { separate the chart from the margins) }\end{array}$ & 3 & - & 2 \\
\hline IAP & Geography & Parallels and Meridians & 3 & - & 3 \\
\hline IAP & Geography & Range & 1 & 4 & 2 \\
\hline IAP & Geography & Spot Elevations & 2 & 2 & $\begin{array}{c}\text { Not Yet } \\
\text { Determined }\end{array}$ \\
\hline IAP & Geography & Terrain Contour Elevations & 2 & 2 & 1 \\
\hline IAP & Geography & Terrain Contours & 2 & 3 & 1 \\
\hline IAP & Geography & $\begin{array}{l}\text { Visual Landmark Label (when not } \\
\text { required for navigation) }\end{array}$ & 2 & 2 & 3 \\
\hline IAP & Geography & $\begin{array}{l}\text { Visual Landmarks (when not required } \\
\text { for navigation) }\end{array}$ & 2 & 4 & 2 \\
\hline IAP & Geography & Water Features & 2 & - & 2 \\
\hline IAP & Holding Pattern & Holding Pattern Altitude & 1 & 1.2 & 1 \\
\hline IAP & Holding Pattern & Holding Pattern Courses & 2 & 1 & 1 \\
\hline IAP & Holding Pattern & Holding Pattern Depiction & 1 & 1 & 1 \\
\hline IAP & Holding Pattern & Holding Pattern Leg Length & 2 & 2 & 2 \\
\hline IAP & Holding Pattern & Holding Pattern Speed & 2 & 2 & 2 \\
\hline IAP & Holding Pattern & Holding Pattern Time & 2 & 1 & 2 \\
\hline IAP & Identification & Airport Elevation & 1 & 1 & 1 \\
\hline IAP & Identification & Airport ICAO Identifier & 1 & 1 & 1 \\
\hline IAP & Identification & Airport Name & 1 & 1 & 1 \\
\hline IAP & Identification & Changes & 3 & - & 1 \\
\hline IAP & Identification & Chart Index Number/Page Number & 3 & - & 2 \\
\hline IAP & Identification & City/Location Name & - & 2,4 & 2 \\
\hline IAP & Identification & Effective Date & 3 & - & 2 \\
\hline IAP & Identification & Procedure Name & 1 & 1 & 1 \\
\hline IAP & Identification & Revision Date & 3 & - & 2 \\
\hline IAP & Landing Minimums & CAT I Decision Altitude (DA) & 1 & 1 & 1 \\
\hline IAP & Landing Minimums & CAT II Decision Altitude (DA) & 2 & 1 & 1 \\
\hline IAP & Landing Minimums & CAT II Radio Altimeter (RA) & 1 & 1 & 1 \\
\hline IAP & Landing Minimums & Decision Height (DH) & 2 & 1 & 1 \\
\hline IAP & Landing Minimums & Height Above Airport (HAA) & 2 & 1 & 1 \\
\hline IAP & Landing Minimums & Minimum Descent Altitude (MDA) & 1 & 1 & 1 \\
\hline IAP & Landing Minimums & Minimum Descent Height (MDH) & 2 & 1 & 1 \\
\hline IAP & Landing Minimums & Visibility Requirement & 1 & 1 & 1 \\
\hline IAP & $\begin{array}{l}\text { Minimum Area/Sector } \\
\text { Altitudes }\end{array}$ & Minimum Radar Altitudes and Sectors & 3 & - & 2 \\
\hline
\end{tabular}




\begin{tabular}{|c|c|c|c|c|c|}
\hline $\begin{array}{l}\text { Chart } \\
\text { Type }\end{array}$ & Information Category & Information Element & $\begin{array}{c}\text { SAE ARP } \\
5621 \\
(2011) \\
\end{array}$ & $\begin{array}{l}\text { Pepitone } \\
\text { et al. } \\
(2014)\end{array}$ & $\begin{array}{l}\text { Current } \\
\text { Survey }\end{array}$ \\
\hline IAP & $\begin{array}{l}\text { Minimum Area/Sector } \\
\text { Altitudes }\end{array}$ & MSA Distance when other than $25 \mathrm{~nm}$ & 2 & 2 & 2 \\
\hline IAP & $\begin{array}{l}\text { Minimum Area/Sector } \\
\text { Altitudes }\end{array}$ & MSA Minimum Altitudes & 2 & 2 & 1 \\
\hline IAP & $\begin{array}{l}\text { Minimum Area/Sector } \\
\text { Altitudes }\end{array}$ & MSA Reference Point/Center & 2 & 2 & 2 \\
\hline IAP & $\begin{array}{l}\text { Minimum Area/Sector } \\
\text { Altitudes }\end{array}$ & MSA Sector Radials & 2 & 2,3 & 2 \\
\hline IAP & Missed Approach & Distance From FAF to MAP & 1 & 1 & 2 \\
\hline IAP & Missed Approach & Fix Name/Identifier at MAP & 1 & 1 & 1 \\
\hline IAP & Missed Approach & Location of MAP & 1 & 1 & 1 \\
\hline IAP & Missed Approach & Missed Approach Holding Pattern & 3 & - & 1 \\
\hline IAP & Missed Approach & Missed Approach Instructions & 3 & - & 1 \\
\hline IAP & Missed Approach & Name of Missed Approach Holding Fix & 3 & - & 1 \\
\hline IAP & Missed Approach & Time From FAF to MAP & 1 & 1 & $\begin{array}{c}\text { Not Yet } \\
\text { Determined }\end{array}$ \\
\hline IAP & $\begin{array}{l}\text { Navaids in the Vicinity of the } \\
\text { Procedure }\end{array}$ & DME Availability & 3 & - & 2 \\
\hline IAP & $\begin{array}{l}\text { Navaids in the Vicinity of the } \\
\text { Procedure }\end{array}$ & Navaid Class & 3 & - & 3 \\
\hline IAP & $\begin{array}{l}\text { Navaids in the Vicinity of the } \\
\text { Procedure }\end{array}$ & Navaid Frequency & 2 & 3 & $\begin{array}{c}\text { Not Yet } \\
\text { Determined }\end{array}$ \\
\hline IAP & $\begin{array}{l}\text { Navaids in the Vicinity of the } \\
\text { Procedure }\end{array}$ & Navaid Identifier & 1 & 3 & 2 \\
\hline IAP & $\begin{array}{l}\text { Navaids in the Vicinity of the } \\
\text { Procedure }\end{array}$ & Navaid Latitude/Longitude & 3 & - & 3 \\
\hline IAP & $\begin{array}{l}\text { Navaids in the Vicinity of the } \\
\text { Procedure }\end{array}$ & Navaid Morse Code & 3 & - & $\begin{array}{c}\text { Not Yet } \\
\text { Determined }\end{array}$ \\
\hline IAP & $\begin{array}{l}\text { Navaids in the Vicinity of the } \\
\text { Procedure }\end{array}$ & Navaid Name & 2 & 3 & 2 \\
\hline IAP & $\begin{array}{l}\text { Navaids in the Vicinity of the } \\
\text { Procedure }\end{array}$ & Navaid Symbol & 1 & 1 & $\begin{array}{c}\text { Not Yet } \\
\text { Determined }\end{array}$ \\
\hline IAP & Navigation & All appropriate navaid symbols & 1 & 1 & 1 \\
\hline IAP & Navigation & FAF (e.g., Maltese Cross) & 1 & 1 & 1 \\
\hline IAP & Navigation & FAF Crossing Altitude (MSL) (HAT) & 1 & 1 & 1 \\
\hline IAP & Navigation & FIR/UIR Boundaries & 3 & - & 2 \\
\hline IAP & Navigation & Fix Altitude & 1 & 1 & 1 \\
\hline IAP & Navigation & Fix Formation & 1 & 1 & 1 \\
\hline IAP & Navigation & Fix Name/Identifier & 1 & 1 & 1 \\
\hline IAP & Navigation & Fix Symbol & 1 & 1 & 1 \\
\hline IAP & Navigation & General Notes & 2 & 3,4 & 2 \\
\hline IAP & Navigation & Glide Slope Angle & 2 & 1 & 2 \\
\hline IAP & Navigation & $\begin{array}{l}\text { GS Intercept Altitude (Above Airport) } \\
\text { (QFE) }\end{array}$ & 1 & 1 & 1 \\
\hline IAP & Navigation & GS Intercept Altitude (MSL) & 1 & 1 & 1 \\
\hline
\end{tabular}




\begin{tabular}{|c|c|c|c|c|c|}
\hline $\begin{array}{l}\text { Chart } \\
\text { Type }\end{array}$ & Information Category & Information Element & $\begin{array}{c}\text { SAE ARP } \\
5621 \\
(2011) \\
\end{array}$ & $\begin{array}{l}\text { Pepitone } \\
\text { et al. } \\
(2014)\end{array}$ & $\begin{array}{l}\text { Current } \\
\text { Survey }\end{array}$ \\
\hline IAP & Navigation & Lead Radial & 1 & 1 & 1 \\
\hline IAP & Navigation & Localizer Magnetic Course & 1 & 1 & 1 \\
\hline IAP & Navigation & Procedural Data Notes & 2 & 2 & 2 \\
\hline IAP & Navigation & Procedure Magnetic Course & 1 & 1 & 1 \\
\hline IAP & Navigation & Procedure Track & 1 & 1 & 1 \\
\hline IAP & Navigation & Procedure Track Altitude & 1 & 1 & 1 \\
\hline IAP & Navigation & Procedure Track Mileage & 1 & 1 & 1 \\
\hline IAP & Navigation & Procedure Turn Altitude & 1 & 2 & 1 \\
\hline IAP & Navigation & Procedure Turn Distance Limit & 1 & 1 & 1 \\
\hline IAP & Navigation & Procedure Turn Outbound Course & 1 & 1 & 1 \\
\hline IAP & Navigation & $\begin{array}{l}\text { Prohibited, Restricted and Danger } \\
\text { Airspace Graphic }\end{array}$ & 1 & 3 & 1 \\
\hline IAP & Navigation & $\begin{array}{l}\text { Prohibited, Restricted and Danger } \\
\text { Airspace Label }\end{array}$ & 3 & - & 1 \\
\hline IAP & Navigation & $\begin{array}{l}\text { Prohibited, Restricted and Danger } \\
\text { Airspace Narrative }\end{array}$ & 3 & - & 2 \\
\hline IAP & Navigation & Rate of Descent (feet per minute) & 2 & 2 & 2 \\
\hline IAP & Navigation & Special Use Airspace - Other & 2 & 3 & 2 \\
\hline IAP & Navigation & Step-Down Fix Altitude & 1 & 1 & 1 \\
\hline IAP & Navigation & Step-Down Fix Formation & 1 & 1 & 1 \\
\hline IAP & Navigation & Terminal Arrival Area (TAA) & 2 & 1 & 2 \\
\hline IAP & Navigation & Threshold Crossing Height & 2 & 2 & 2 \\
\hline IAP & Navigation & Transition Altitude & 2 & 2 & 2 \\
\hline IAP & Navigation & Transition Level & 2 & 2 & 2 \\
\hline IAP & Navigation & VNAV Angle & 2 & 1 & 2 \\
\hline IAP & Navigation & $\begin{array}{l}\text { VNAV Intercept Altitude (Above } \\
\text { Airport) (QFE) }\end{array}$ & 1 & 1,2 & 1 \\
\hline IAP & Navigation & VNAV Intercept Altitude (MSL) & 1 & 1 & 1 \\
\hline IAP & Navigation Aids & Localizer for Intersection Formations & 1 & 1 & 2 \\
\hline IAP & Navigation Aids & Localizer Frequency & 1 & 1 & 1 \\
\hline IAP & Navigation Aids & $\begin{array}{l}\text { Localizer Front Course for Back } \\
\text { Course Approaches }\end{array}$ & 1 & 1 & $\begin{array}{c}\text { Not Yet } \\
\text { Determined }\end{array}$ \\
\hline IAP & Navigation Aids & Localizer Identifier & - & 1 & 2 \\
\hline IAP & Navigation Aids & Localizer Morse Code & - & 2,3 & $\begin{array}{c}\text { Not Yet } \\
\text { Determined }\end{array}$ \\
\hline IAP & Navigation Aids & $\begin{array}{l}\text { Marker Beacon Labels (i.e., } \\
\text { OM,MM,IM) }\end{array}$ & 1 & 1 & 2 \\
\hline IAP & Navigation Aids & Marker Beacon Symbols & 1 & 2 & 2 \\
\hline IAP & Navigation Aids & Primary Approach Localizer Symbol & 1 & 1 & 2 \\
\hline IAP & Navigation Aids & $\begin{array}{l}\text { Simultaneous Parallel Localizer } \\
\text { Symbol }\end{array}$ & - & 2 & 2 \\
\hline IAP & Navigation Aids & WAAS/SBAS -LAAS/GBAS Channel & 1 & 2 & 2 \\
\hline
\end{tabular}




\begin{tabular}{|c|l|l|c|c|c|}
\hline $\begin{array}{c}\text { Chart } \\
\text { Type }\end{array}$ & Information Category & Information Element & $\begin{array}{c}\text { SAE ARP } \\
\mathbf{5 6 2 1} \\
\mathbf{( 2 0 1 1}\end{array}$ & $\begin{array}{c}\text { Pepitone } \\
\text { et al. } \\
\mathbf{( 2 0 1 4}\end{array}$ & $\begin{array}{c}\text { Current } \\
\text { Survey }\end{array}$ \\
\hline IAP & Obstacles & Obstacle Heights and related datum & 2 & 3 & 1 \\
\hline IAP & Obstacles & Obstacle Symbols and Elevation & 2 & - & 1 \\
\hline IAP & Primary Airport & Glide Path Intercept Point & 3 & - & 1 \\
\hline IAP & Primary Airport & Landing Runway Number & 1 & 1 & 1 \\
\hline IAP & Primary Airport & Other Runway Numbers & 3 & - & 2 \\
\hline IAP & Primary Airport & Runway Layouts & 1 & 1 & 1 \\
\hline IAP & Primary Airport & Runway Location in Profile View & 1 & 1 & 1 \\
\hline IAP & Primary Airport & Straight-in Landing Runway Length & 3 & - & $\begin{array}{c}\text { Not Yet } \\
\text { Determined }\end{array}$ \\
\hline IAP & Primary Airport & $\begin{array}{l}\text { TDZE/Threshold Elevation for Landing } \\
\text { Runway }\end{array}$ & 1 & 1 & 1 \\
\hline IAP & Procedure Navaid & DME Availability & 3 & - & 2 \\
\hline IAP & Procedure Navaid & Navaid Class & 2 & - & 3 \\
\hline IAP & Procedure Navaid & Navaid Frequency & 1 & 2 & 2 \\
\hline IAP & Procedure Navaid & Navaid Identifier & 3 & - & 3 \\
\hline IAP & Procedure Navaid & Navaid Latitude/Longitude & 3 & - & $\begin{array}{c}\text { Not Yet } \\
\text { Determined }\end{array}$ \\
\hline IAP & Procedure Navaid & Navaid Morse Code & 2 & - & 1 \\
\hline IAP & Procedure Navaid & Navaid Name & 2 & 3 & 1 \\
\hline IAP & Procedure Navaid & Navaid Symbol & - & 3 & 2 \\
\hline IAP & Secondary Airports & IFR Airports in Plan View & 3 & $\begin{array}{c}\text { Not Yet } \\
\text { Determined }\end{array}$ \\
\hline IAP & Secondary Airports & $\begin{array}{l}\text { Source Doc-Runway Layouts and } \\
\text { Name }\end{array}$ & $\begin{array}{c}\text { Not Yet } \\
\text { Determined }\end{array}$ \\
\hline IAP & Secondary Airports & DFR Airports within Specified & 3 & & 1 \\
\hline
\end{tabular}

Table 5

Survey Results for Enroute Instrument Flight Rules (IFR) Charts

\begin{tabular}{|c|l|l|c|c|c|}
\hline $\begin{array}{c}\text { Chart } \\
\text { Type }\end{array}$ & Information & Information Element & $\begin{array}{c}\text { SAE ARP } \\
\mathbf{5 6 2 1} \\
\mathbf{( 2 0 1 1 )}\end{array}$ & $\begin{array}{c}\text { Pepitone } \\
\text { et al. } \\
\mathbf{( 2 0 1 4 )}\end{array}$ & $\begin{array}{c}\text { Current } \\
\text { Survey }\end{array}$ \\
\hline IFR & Airport Information & Airport Attributes if for IFR use & 2 & - & 2 \\
\hline IFR & Airport Information & Airport Attributes if for VFR use & 3 & - & 2 \\
\hline IFR & Airport Information & Airport Identifier if for IFR use & 2 & - & 1 \\
\hline IFR & Airport Information & Airport Identifier if for VFR use & 3 & - & 2 \\
\hline IFR & Airport Information & $\begin{array}{l}\text { Airport Symbol if for IFR use } \\
\text { (includes suitable symbol type) }\end{array}$ & 2 & - & 1 \\
\hline IFR & Airport Information & $\begin{array}{l}\text { Airport Symbol if for VFR use } \\
\text { (includes suitable symbol type) }\end{array}$ & 3 & - & 1 \\
\hline IFR & Airport Information & Airspace Class Boundaries & 2 & - & 1 \\
\hline IFR & Airport Information & Airspace Class Name of Call Sign & 2 & - & 2 \\
\hline
\end{tabular}




\begin{tabular}{|c|c|c|c|c|c|}
\hline $\begin{array}{l}\text { Chart } \\
\text { Type }\end{array}$ & $\begin{array}{l}\text { Information } \\
\text { Category }\end{array}$ & Information Element & $\begin{array}{c}\text { SAE ARP } \\
5621 \\
(2011) \\
\end{array}$ & $\begin{array}{c}\text { Pepitone } \\
\text { et al. } \\
\text { (2014) } \\
\end{array}$ & $\begin{array}{l}\text { Current } \\
\text { Survey }\end{array}$ \\
\hline IFR & Airport Information & Airspace Class Type & 2 & - & $\begin{array}{c}\text { Not Yet } \\
\text { Determined }\end{array}$ \\
\hline IFR & Airport Information & FIR/UIR Vertical Limits & 2 & - & $\begin{array}{c}\text { Not Yet } \\
\text { Determined }\end{array}$ \\
\hline IFR & Airport Information & $\begin{array}{l}\text { Terminal Control Area (TMA) } \\
\text { Boundaries }\end{array}$ & 2 & - & 2 \\
\hline IFR & Airport Information & $\begin{array}{l}\text { Unit Providing Approach Control } \\
\text { Service }\end{array}$ & 2 & - & 2 \\
\hline IFR & Airspace Boundaries & $\begin{array}{l}\text { Air Defense Identification Zones } \\
\text { (ADIZ) }\end{array}$ & 1 & - & 1 \\
\hline IFR & Airspace Boundaries & Airspace Class Notes & 2 & - & 2 \\
\hline IFR & Airspace Boundaries & Airspace Class Vertical Limits & 2 & - & 2 \\
\hline IFR & Airspace Boundaries & Airway Designator & $2 *$ & - & 1 \\
\hline IFR & Airspace Boundaries & $\begin{array}{l}\text { Altimeter Setting Regions (i.e., } \\
\text { lowest ALT for QNH) }\end{array}$ & 2 & - & 2 \\
\hline IFR & Airspace Boundaries & $\begin{array}{l}\text { Computer Navigation Fix (CNF) and } \\
\text { ID }\end{array}$ & $2^{*}$ & - & 2 \\
\hline IFR & Airspace Boundaries & Control Zone (CTR) Boundaries & 2 & - & 2 \\
\hline IFR & Airspace Boundaries & CTR Vertical Limits & 2 & - & $\begin{array}{c}\text { Not Yet } \\
\text { Determined }\end{array}$ \\
\hline IFR & Airspace Boundaries & FIR/UIR Boundaries & 1 & - & $\begin{array}{c}\text { Not Yet } \\
\text { Determined }\end{array}$ \\
\hline IFR & Airspace Boundaries & Holding Pattern restrictions & $2 *$ & - & 2 \\
\hline IFR & Airspace Boundaries & ID of FIR/UIR & 2 & - & 2 \\
\hline IFR & Airspace Boundaries & $\begin{array}{l}\text { Indication of Areas of RNP, RVSM, } \\
\text { MNPS, etc., Requirements }\end{array}$ & 2 & - & 2 \\
\hline IFR & Airspace Boundaries & $\begin{array}{l}\text { Indication of MEA Change at } \\
\text { Segment End }\end{array}$ & 2 & - & 2 \\
\hline IFR & Airspace Boundaries & Indication of MET Report Required & $2 *$ & - & 2 \\
\hline IFR & Airspace Boundaries & Indication of one-way airways & $2 *$ & - & 1 \\
\hline IFR & Airspace Boundaries & $\begin{array}{l}\text { Intersection, Waypoint, or Fix } \\
\text { Coordinates }\end{array}$ & 2 & - & 3 \\
\hline IFR & Airspace Boundaries & $\begin{array}{l}\text { Intersection, Waypoint, or Fix ID of } \\
\text { VOR, FREQ, MAG BRG }\end{array}$ & $2 *$ & - & 1 \\
\hline IFR & Airspace Boundaries & $\begin{array}{l}\text { Intersection, Waypoint, or Fix } \\
\text { Symbol }\end{array}$ & $2^{*}$ & - & 1 \\
\hline IFR & Airspace Boundaries & Minimum Reception Altitude (MRA) & $2 *$ & - & 2 \\
\hline IFR & Airspace Boundaries & Name of CTR & 2 & - & 2 \\
\hline IFR & Airspace Boundaries & Name of FIR/UIR & 2 & - & 2 \\
\hline IFR & Airspace Boundaries & Name of TMA & 2 & - & 2 \\
\hline IFR & Airspace Boundaries & Procedural Data Notes & 2* & - & 2 \\
\hline IFR & Airspace Boundaries & Segment Mileages & $2^{*}$ & - & $\begin{array}{c}\text { Not Yet } \\
\text { Determined }\end{array}$ \\
\hline IFR & Airspace Boundaries & Segment MORA & $2 *$ & - & 2 \\
\hline IFR & Airspace Boundaries & Segment Upper Limit or MAA & $2 *$ & - & 2 \\
\hline
\end{tabular}




\begin{tabular}{|c|c|c|c|c|c|}
\hline $\begin{array}{l}\text { Chart } \\
\text { Type }\end{array}$ & $\begin{array}{l}\text { Information } \\
\text { Category }\end{array}$ & Information Element & $\begin{array}{c}\text { SAE ARP } \\
5621 \\
(2011) \\
\end{array}$ & $\begin{array}{l}\text { Pepitone } \\
\text { et al. } \\
(2014)\end{array}$ & $\begin{array}{l}\text { Current } \\
\text { Survey }\end{array}$ \\
\hline IFR & Airspace Boundaries & $\begin{array}{l}\text { Special Use Airspace - Other - } \\
\text { Boundaries }\end{array}$ & 2 & - & $\begin{array}{c}\text { Not Yet } \\
\text { Determined }\end{array}$ \\
\hline IFR & Airspace Boundaries & $\begin{array}{l}\text { Special Use Airspace - Prohibited, } \\
\text { Restricted, Danger Boundaries }\end{array}$ & 2 & - & 1 \\
\hline IFR & Airspace Boundaries & $\begin{array}{l}\text { Special Use Airspace ID and Vertical } \\
\text { limits }\end{array}$ & 2 & - & 1 \\
\hline IFR & Airspace Boundaries & Time Zone Boundaries & 3 & - & 3 \\
\hline IFR & Airspace Boundaries & TMA Vertical Limits & 2 & - & 2 \\
\hline IFR & Airspace Boundaries & Unit Providing Area Control Service & 2 & - & 2 \\
\hline IFR & Airspace Boundaries & Unit Providing Service & 2 & - & 2 \\
\hline IFR & Airways & Airway Magnetic Course & $2 *$ & - & 1 \\
\hline IFR & Airways & Airway Symbol (center line) & $2 *$ & - & 1 \\
\hline IFR & Airways & $\begin{array}{l}\text { Fix Formation bearing, frequency, ID } \\
\text { of Remote Navaid }\end{array}$ & $2 *$ & - & 2 \\
\hline IFR & Airways & General Notes & 2 & - & 2 \\
\hline IFR & Airways & Holding Patterns & $2 *$ & - & 1 \\
\hline IFR & Airways & Indication of compulsory reporting & $2 *$ & - & 1 \\
\hline IFR & Airways & $\begin{array}{l}\text { Intersection, Waypoint, or Fix } \\
\text { Distance from Reference DME }\end{array}$ & $2 *$ & - & 1 \\
\hline IFR & Airways & Intersection, Waypoint, or Fix Name & $2 *$ & - & 1 \\
\hline IFR & Airways & Minimum Crossing Altitude (MCA) & $2 *$ & - & 1 \\
\hline IFR & Airways & $\begin{array}{l}\text { Segment Minimum Cruising Level or } \\
\text { MEA }\end{array}$ & $2^{*}$ & - & 1 \\
\hline IFR & Airways & Segment MOCA & $2^{*}$ & - & $\begin{array}{c}\text { Not Yet } \\
\text { Determined }\end{array}$ \\
\hline IFR & Airways & Times of one-way direction & 2* & - & 2 \\
\hline IFR & Airways & Total Distance Between Navaids & 2 & - & $\begin{array}{c}\text { Not Yet } \\
\text { Determined }\end{array}$ \\
\hline IFR & Airways & Transition Text & $2 *$ & - & 2 \\
\hline IFR & Airways & $\begin{array}{l}\text { VOR Change Over Point with } \\
\text { Distances }\end{array}$ & $2 *$ & - & $\begin{array}{c}\text { Not Yet } \\
\text { Determined }\end{array}$ \\
\hline IFR & Communications & $\begin{array}{l}\text { Call and Frequencies of In-Flight } \\
\text { Weather Stations }\end{array}$ & 2 & - & 3 \\
\hline IFR & Communications & $\begin{array}{l}\text { Company Specific Frequencies } \\
\text { (tailored communications) }\end{array}$ & 2 & - & 3 \\
\hline IFR & Communications & $\begin{array}{l}\text { FIR/UIR, Control, ARTCC, etc., } \\
\text { Frequency Boxes }\end{array}$ & 2 & - & 2 \\
\hline IFR & Communications & $\begin{array}{l}\text { Graphical Portrayal of Radio } \\
\text { Frequency Sector Boundaries }\end{array}$ & 2 & - & 2 \\
\hline IFR & Communications & $\begin{array}{l}\text { Voice Frequencies associated with } \\
\text { Navaid Facility Boxes }\end{array}$ & 2 & - & 2 \\
\hline IFR & Geography & Contour Interval Legend & 3 & - & $\begin{array}{c}\text { Not Yet } \\
\text { Determined }\end{array}$ \\
\hline IFR & Geography & Indication of Area Chart Coverage & 3 & - & 2 \\
\hline IFR & Geography & $\begin{array}{l}\text { International Boundaries (higher } \\
\text { criticality where appropriate) }\end{array}$ & 2 & - & 1 \\
\hline
\end{tabular}




\begin{tabular}{|c|c|c|c|c|c|}
\hline $\begin{array}{l}\text { Chart } \\
\text { Type }\end{array}$ & $\begin{array}{l}\text { Information } \\
\text { Category }\end{array}$ & Information Element & $\begin{array}{c}\text { SAE ARP } \\
5621 \\
(2011) \\
\end{array}$ & $\begin{array}{c}\text { Pepitone } \\
\text { et al. } \\
\text { (2014) }\end{array}$ & $\begin{array}{l}\text { Current } \\
\text { Survey }\end{array}$ \\
\hline IFR & Geography & Parallels and Meridians & 1 & - & 2 \\
\hline IFR & Geography & Range & 1 & - & 2 \\
\hline IFR & Geography & Spot Elevations & 3 & - & 2 \\
\hline IFR & Geography & Terrain Contour Elevations & 3 & - & 2 \\
\hline IFR & Geography & Terrain Contours & 3 & - & 2 \\
\hline IFR & Geography & Water Features & 2 & - & 2 \\
\hline IFR & Identification & $\begin{array}{l}\text { Chart Description (e.g., High, Low, } \\
\text { etc.) }\end{array}$ & 3 & - & 2 \\
\hline IFR & Identification & Revision Date (i.e., Start and Finish) & 3 & - & 2 \\
\hline IFR & Minimum Area/Sector Altitudes & $\begin{array}{l}\text { Area Minimum Altitudes - OROCA, } \\
\text { Sector Altitudes (Grid MORA } \\
\text { Outside of US) }\end{array}$ & 1 & - & 1 \\
\hline IFR & Navigation Aids & Broadcast Stations or Marine Beacons & 3 & - & 3 \\
\hline IFR & Navigation Aids & DME Antenna Elevation & 3 & - & 3 \\
\hline IFR & Navigation Aids & Indication of True North Navaids & 2 & - & 3 \\
\hline IFR & Navigation Aids & Navaid Class (e.g., H, T, and L) & 3 & - & 3 \\
\hline IFR & Navigation Aids & Navaid Coordinates & 3 & - & 3 \\
\hline IFR & Navigation Aids & Navaid Frequency & $2^{*}$ & - & 1 \\
\hline IFR & Navigation Aids & Navaid Identifier & $2 *$ & - & 1 \\
\hline IFR & Navigation Aids & Navaid Morse Code & 2 & - & 2 \\
\hline IFR & Navigation Aids & Navaid Name & $2 *$ & - & 1 \\
\hline IFR & Navigation Aids & Navaid Station Declination & 3 & - & 3 \\
\hline IFR & Navigation Aids & Navaid Symbol & 1 & - & 1 \\
\hline IFR & Navigation Aids & Notes on Navaid Operational Status & 2 & - & 2 \\
\hline IFR & Obstacle & $\begin{array}{l}\text { Obstacle Symbols and Elevation (e.g., } \\
\text { man-made, exceptionally high) }\end{array}$ & 3 & - & 1 \\
\hline
\end{tabular}

\section{Table 6}

Survey Results for Standard Instrument Departure (SID) Charts

\begin{tabular}{|c|l|l|c|c|c|}
\hline $\begin{array}{c}\text { Chart } \\
\text { Type }\end{array}$ & $\begin{array}{l}\text { Information } \\
\text { Category }\end{array}$ & Information Element & $\begin{array}{c}\text { SAE ARP } \\
\mathbf{5 6 2 1} \\
\mathbf{( 2 0 1 1 )}\end{array}$ & $\begin{array}{c}\text { Pepitone } \\
\text { et al. } \\
\mathbf{( 2 0 1 4 )}\end{array}$ & $\begin{array}{c}\text { Current } \\
\text { Survey }\end{array}$ \\
\hline SID & Airport Information & Airport Elevation & 1 & 1 & 1 \\
\hline SID & Airport Information & Distances from airport to first fix on SID & 3 & - & 1 \\
\hline SID & Airport Information & Other Airport Elevations & 2 & 4 & $\begin{array}{c}\text { Not Yet } \\
\text { Determined }\end{array}$ \\
\hline SID & Airport Information & Other Airport Names & 2 & 3 & $\begin{array}{c}\text { Not Yet } \\
\text { Determined }\end{array}$ \\
\hline SID & Airport Information & Other Airport Symbols & 2 & 3 & 2 \\
\hline SID & Airport Information & Runway Layout & 1 & 2 & 1 \\
\hline
\end{tabular}




\begin{tabular}{|c|c|c|c|c|c|}
\hline $\begin{array}{l}\text { Chart } \\
\text { Type }\end{array}$ & $\begin{array}{l}\text { Information } \\
\text { Category }\end{array}$ & Information Element & $\begin{array}{c}\text { SAE ARP } \\
5621 \\
(2011) \\
\end{array}$ & $\begin{array}{c}\text { Pepitone } \\
\text { et al. } \\
(2014)\end{array}$ & $\begin{array}{l}\text { Current } \\
\text { Survey }\end{array}$ \\
\hline SID & Communications & Communications Boundaries & 2 & 3 & 2 \\
\hline SID & Communications & Departure Control Frequency & 2 & 2 & 1 \\
\hline SID & Communications & Lost Comm Procedure & 2 & 4 & 2 \\
\hline SID & Communications & Lost Comm Procedure Outline Lines & 2 & 3 & 2 \\
\hline SID & Communications & Transponder Setting where appropriate & 2 & 2,3 & 2 \\
\hline SID & Course Definition & Heading & 1 & 1 & 1 \\
\hline SID & Course Definition & MEA/MOCA & 1 & 3 & 1 \\
\hline SID & Course Definition & Radial & 1 & 1 & 1 \\
\hline SID & Course Definition & Segment Mileages & 2 & 1,2 & 1 \\
\hline SID & Course Definition & Track & 1 & 1 & 1 \\
\hline SID & Course Definition & VOR Change Over Point & 2 & 2,3 & 2 \\
\hline SID & Geography & Contour Interval Legend & 3 & - & $\begin{array}{c}\text { Not Yet } \\
\text { Determined }\end{array}$ \\
\hline SID & Geography & Cultural Features & 3 & - & $\begin{array}{c}\text { Not Yet } \\
\text { Determined }\end{array}$ \\
\hline SID & Geography & $\begin{array}{l}\text { Highest Reference Point (within neat } \\
\text { lines) }\end{array}$ & 2 & 3 & 1 \\
\hline SID & Geography & $\begin{array}{l}\text { International Boundaries (higher } \\
\text { criticality where appropriate) }\end{array}$ & 2 & 3 & 2 \\
\hline SID & Geography & $\begin{array}{l}\text { Neat Lines (i.e., the lines which separate } \\
\text { the chart from the margins) }\end{array}$ & 3 & - & 2 \\
\hline SID & Geography & Parallels and Meridians & 2 & 3 & 3 \\
\hline SID & Geography & $\begin{array}{l}\text { Parallels and Meridians with AMAs, } \\
\text { OROCAs, MORAs }\end{array}$ & 2 & - & $\begin{array}{c}\text { Not Yet } \\
\text { Determined }\end{array}$ \\
\hline SID & Geography & Range & 1 & 2 & $\begin{array}{c}\text { Not Yet } \\
\text { Determined }\end{array}$ \\
\hline SID & Geography & Spot Elevations & 2 & 2 & 1 \\
\hline SID & Geography & Terrain Contour Elevations & 2 & 2 & 1 \\
\hline SID & Geography & Terrain Contours & 2 & 2 & 1 \\
\hline SID & Geography & Water Features & 3 & - & $\begin{array}{c}\text { Not Yet } \\
\text { Determined }\end{array}$ \\
\hline SID & Holding Pattern & Holding Pattern Altitude & 1 & 1 & 1 \\
\hline SID & Holding Pattern & Holding Pattern Courses & 2 & 2 & 1 \\
\hline SID & Holding Pattern & Holding Pattern Depiction & 1 & 2 & 1 \\
\hline SID & Holding Pattern & Holding Pattern Leg Length & 2 & 2 & 1 \\
\hline SID & Holding Pattern & Holding Pattern Speed & 2 & 2 & 2 \\
\hline SID & Holding Pattern & Holding Pattern Time & 2 & 2,3 & 2 \\
\hline SID & Identification & Airport ICAO Identifier & 1 & 1 & 1 \\
\hline SID & Identification & Airport Name & 1 & 1 & 1 \\
\hline SID & Identification & Changes & 3 & - & 2 \\
\hline SID & Identification & Chart Index Number/Page Number & 3 & - & 2 \\
\hline SID & Identification & City/Location Name & 2 & 3 & $\begin{array}{c}\text { Not Yet } \\
\text { Determined }\end{array}$ \\
\hline
\end{tabular}




\begin{tabular}{|c|c|c|c|c|c|}
\hline $\begin{array}{l}\text { Chart } \\
\text { Type }\end{array}$ & $\begin{array}{l}\text { Information } \\
\text { Category }\end{array}$ & Information Element & $\begin{array}{c}\text { SAE ARP } \\
5621 \\
(2011)\end{array}$ & $\begin{array}{l}\text { Pepitone } \\
\text { et al. } \\
(2014)\end{array}$ & $\begin{array}{l}\text { Current } \\
\text { Survey }\end{array}$ \\
\hline SID & Identification & $\begin{array}{l}\text { DP Type (e.g., Pilot Nav, Vector, Noise, } \\
\text { Obstacle) }\end{array}$ & 2 & $1,2,3,4$ & 2 \\
\hline SID & Identification & Effective Date & 3 & - & 2 \\
\hline SID & Identification & $\begin{array}{l}\text { Procedure Identifier (e.g., } \\
\text { CNOG8.VNY+A42) }\end{array}$ & 2 & 1 & 1 \\
\hline SID & Identification & Procedure Name (e.g., Canoga Eight) & 1 & 1 & 1 \\
\hline SID & Identification & Revision Date & 3 & - & 2 \\
\hline SID & $\begin{array}{l}\text { Instrument Procedure } \\
\text { Courses/Tracks }\end{array}$ & Identifier (i.e., CNOG8.AVE) & 1 & - & 1 \\
\hline SID & $\begin{array}{l}\text { Instrument Procedure } \\
\text { Courses/Tracks }\end{array}$ & Symbol (e.g., line style, etc.) & 1 & - & 1 \\
\hline SID & $\begin{array}{l}\text { Intersection /Fixes on } \\
\text { Procedure }\end{array}$ & Identifier & 1 & 1 & 1 \\
\hline SID & $\begin{array}{l}\text { Intersection /Fixes on } \\
\text { Procedure }\end{array}$ & Latitude/Longitudes & 3 & - & 3 \\
\hline SID & $\begin{array}{l}\text { Intersection /Fixes on } \\
\text { Procedure }\end{array}$ & MRA & 3 & - & 2 \\
\hline SID & $\begin{array}{l}\text { Intersection /Fixes on } \\
\text { Procedure }\end{array}$ & Names & 2 & 1 & 1 \\
\hline SID & $\begin{array}{l}\text { Intersection /Fixes on } \\
\text { Procedure }\end{array}$ & Symbol & 1 & 2 & 1 \\
\hline SID & $\begin{array}{l}\text { Minimum Area/Sector } \\
\text { Altitudes }\end{array}$ & $\begin{array}{l}\text { AMA, OROCA, or grid MORA where } \\
\text { established }\end{array}$ & 2 & 3 & 2 \\
\hline SID & $\begin{array}{l}\text { Minimum Area/Sector } \\
\text { Altitudes }\end{array}$ & Minimum Radar Altitudes and Sectors & 3 & - & 1 \\
\hline SID & $\begin{array}{l}\text { Minimum Area/Sector } \\
\text { Altitudes }\end{array}$ & MSA Distance when other than 25nm & 2 & 3 & 2 \\
\hline SID & $\begin{array}{l}\text { Minimum Area/Sector } \\
\text { Altitudes }\end{array}$ & MSA Minimum Altitudes & 2 & 2 & 1 \\
\hline SID & $\begin{array}{l}\text { Minimum Area/Sector } \\
\text { Altitudes }\end{array}$ & MSA Reference Point/Center & 2 & 2,3 & $\begin{array}{c}\text { Not Yet } \\
\text { Determined }\end{array}$ \\
\hline SID & $\begin{array}{l}\text { Minimum Area/Sector } \\
\text { Altitudes }\end{array}$ & MSA Sector Radials & 2 & 3 & 1 \\
\hline SID & $\begin{array}{l}\text { Navaid Used to Form } \\
\text { Fixes }\end{array}$ & DME Availability (Text information) & 3 & - & 2 \\
\hline SID & $\begin{array}{l}\text { Navaid Used to Form } \\
\text { Fixes }\end{array}$ & DME Distances that form fixes & 2 & 1,3 & 1 \\
\hline SID & $\begin{array}{l}\text { Navaid Used to Form } \\
\text { Fixes }\end{array}$ & Navaid Class & 3 & - & 3 \\
\hline SID & $\begin{array}{l}\text { Navaid Used to Form } \\
\text { Fixes }\end{array}$ & Navaid Frequency/Channel & 2 & 1 & 1 \\
\hline SID & $\begin{array}{l}\text { Navaid Used to Form } \\
\text { Fixes }\end{array}$ & Navaid Identifier & 1 & 1 & 1 \\
\hline SID & $\begin{array}{l}\text { Navaid Used to Form } \\
\text { Fixes }\end{array}$ & Navaid Latitude/Longitude & 3 & - & 3 \\
\hline SID & $\begin{array}{l}\text { Navaid Used to Form } \\
\text { Fixes }\end{array}$ & Navaid Morse Code & 2 & 3 & $\begin{array}{c}\text { Not Yet } \\
\text { Determined }\end{array}$ \\
\hline SID & $\begin{array}{l}\text { Navaid Used to Form } \\
\text { Fixes }\end{array}$ & Navaid Name & 2 & 1 & 1 \\
\hline
\end{tabular}




\begin{tabular}{|c|c|c|c|c|c|}
\hline $\begin{array}{l}\text { Chart } \\
\text { Type }\end{array}$ & $\begin{array}{l}\text { Information } \\
\text { Category }\end{array}$ & Information Element & $\begin{array}{c}\text { SAE ARP } \\
5621 \\
(2011) \\
\end{array}$ & $\begin{array}{c}\text { Pepitone } \\
\text { et al. } \\
\text { (2014) }\end{array}$ & $\begin{array}{l}\text { Current } \\
\text { Survey }\end{array}$ \\
\hline SID & $\begin{array}{l}\text { Navaid Used to Form } \\
\text { Fixes }\end{array}$ & Navaid Radials/Bearings that form fixes & 2 & 1 & 1 \\
\hline SID & $\begin{array}{l}\text { Navaid Used to Form } \\
\text { Fixes }\end{array}$ & Navaid Symbol & 1 & 1,2 & 1 \\
\hline SID & $\begin{array}{l}\text { Navaid Used to Form Leg } \\
\text { of Procedure }\end{array}$ & DME Availability (Text information) & 3 & - & 2 \\
\hline SID & $\begin{array}{l}\text { Navaid Used to Form Leg } \\
\text { of Procedure }\end{array}$ & Navaid Class & 3 & - & 3 \\
\hline SID & $\begin{array}{l}\text { Navaid Used to Form Leg } \\
\text { of Procedure }\end{array}$ & Navaid Frequency/Channel & 2 & 1 & 1 \\
\hline SID & $\begin{array}{l}\text { Navaid Used to Form Leg } \\
\text { of Procedure }\end{array}$ & Navaid Identifier & 1 & 2 & 1 \\
\hline SID & $\begin{array}{l}\text { Navaid Used to Form Leg } \\
\text { of Procedure }\end{array}$ & Navaid Latitude/Longitude & 3 & - & 3 \\
\hline SID & $\begin{array}{l}\text { Navaid Used to Form Leg } \\
\text { of Procedure }\end{array}$ & Navaid Morse Code & 2 & 3 & $\begin{array}{c}\text { Not Yet } \\
\text { Determined }\end{array}$ \\
\hline SID & $\begin{array}{l}\text { Navaid Used to Form Leg } \\
\text { of Procedure }\end{array}$ & Navaid Name & 2 & 1 & 1 \\
\hline SID & $\begin{array}{l}\text { Navaid Used to Form Leg } \\
\text { of Procedure }\end{array}$ & Navaid Symbol & 1 & 1 & 1 \\
\hline SID & Navigation & FIR/UIR Boundaries & 3 & - & 2 \\
\hline SID & Navigation & $\begin{array}{l}\text { Prohibited, Restricted and Danger } \\
\text { Airspace Graphic }\end{array}$ & 1 & - & 1 \\
\hline SID & Navigation & $\begin{array}{l}\text { Prohibited, Restricted and Danger } \\
\text { Airspace Label }\end{array}$ & 3 & - & 1 \\
\hline SID & Navigation & $\begin{array}{l}\text { Prohibited, Restricted and Danger } \\
\text { Airspace Narrative }\end{array}$ & 3 & - & 2 \\
\hline SID & Navigation & Special Use Airspace - Other & 2 & - & $\begin{array}{c}\text { Not Yet } \\
\text { Determined }\end{array}$ \\
\hline SID & Navigation & Transition Altitude & 2 & - & 1 \\
\hline SID & Obstacle & Obstacle Symbols and Elevation & 3 & - & 1 \\
\hline SID & Textual Information & Climb Gradient - ATC & 1 & 4 & 2 \\
\hline SID & Textual Information & Climb Gradient - Obstacle & 1 & 4 & $\begin{array}{c}\text { Not Yet } \\
\text { Determined }\end{array}$ \\
\hline SID & Textual Information & Crossing Altitude Restrictions & 1 & 2 & 1 \\
\hline SID & Textual Information & General Notes & 2 & 4 & 2 \\
\hline SID & Textual Information & Noise Abatement & 2 & 4 & 2 \\
\hline SID & Textual Information & Notes & 2 & 4 & 2 \\
\hline SID & Textual Information & $\begin{array}{l}\text { Performance limitations (e.g., bank } \\
\text { limits) }\end{array}$ & 2 & 4 & 2 \\
\hline SID & Textual Information & Procedural Data Notes & 2 & 3 & 2 \\
\hline SID & Textual Information & Runway departure text & 1 & 4 & $\begin{array}{c}\text { Not Yet } \\
\text { Determined }\end{array}$ \\
\hline SID & Textual Information & Speed restrictions & 1 & 2,3 & 1 \\
\hline SID & Textual Information & Text-Only Procedures & 1 & 3 & 2 \\
\hline SID & Textual Information & Transition Name & 1 & 1 & 1 \\
\hline SID & Textual Information & Transition Text & 3 & - & 2 \\
\hline
\end{tabular}




\begin{tabular}{|c|l|l|c|c|c|}
\hline $\begin{array}{c}\text { Chart } \\
\text { Type }\end{array}$ & $\begin{array}{l}\text { Information } \\
\text { Category }\end{array}$ & Information Element & $\begin{array}{c}\text { SAE ARP } \\
\mathbf{5 6 2 1} \\
\mathbf{( 2 0 1 1}\end{array}$ & $\begin{array}{c}\text { Pepitone } \\
\text { et al. } \\
\mathbf{( 2 0 1 4 )}\end{array}$ & $\begin{array}{c}\text { Current } \\
\text { Survey }\end{array}$ \\
\hline SID & Transitions & Transition Course - Magnetic Values & 1 & 2 & $\begin{array}{c}\text { Not Yet } \\
\text { Determined }\end{array}$ \\
\hline SID & Transitions & $\begin{array}{l}\text { Transition Course notes (e.g., DME } \\
\text { required) }\end{array}$ & 2 & 2,3 & 2 \\
\hline SID & Transitions & Transition Courses computer codes & 2 & 1 & 2 \\
\hline SID & Transitions & Transition Courses depiction & 1 & 1 & 1 \\
\hline SID & Transitions & Transition Courses -MEAs, MOCAs & 2 & 2,3 & $\begin{array}{c}\text { Not Yet } \\
\text { Determined }\end{array}$ \\
\hline SID & Transitions & Transition Courses -segment mileages & 2 & 1 & $\begin{array}{c}\text { Not Yet } \\
\text { Determined }\end{array}$ \\
\hline SID & Transitions & Transition Text & 1 & 2,3 & 2 \\
\hline
\end{tabular}

\section{Table 7}

Survey Results for Standard Terminal Arrival Route (STAR) Charts

\begin{tabular}{|c|c|c|c|c|c|}
\hline $\begin{array}{l}\text { Chart } \\
\text { Type }\end{array}$ & $\begin{array}{l}\text { Information } \\
\text { Category }\end{array}$ & Information Element & $\begin{array}{c}\text { SAE ARP } \\
5621 \\
(2011) \\
\end{array}$ & $\begin{array}{c}\text { Pepitone } \\
\text { et al. } \\
(2014)\end{array}$ & $\begin{array}{c}\text { Current } \\
\text { Survey }\end{array}$ \\
\hline STAR & Airport Information & Distances from last STAR fix to airport & 3 & a & $\begin{array}{c}\text { Not Yet } \\
\text { Determined }\end{array}$ \\
\hline STAR & Airport Information & Other Airport Elevations & 3 & - & 3 \\
\hline STAR & Airport Information & Other Airport Names & 2 & 3 & 2 \\
\hline STAR & Airport Information & Other Airport Symbols & 2 & 3 & 2 \\
\hline STAR & Airport Information & Primary Airport elevation & 1 & 1 & 1 \\
\hline STAR & Airport Information & Primary Airport Runway Layout & 1 & 1,3 & 1 \\
\hline STAR & Airport Information & Primary Airport Shaded Area & 2 & 2,3 & 1 \\
\hline STAR & Communications & ACARS - D - ATIS, TWIP & 3 & - & 2 \\
\hline STAR & Communications & Approach Control (Arrival) & 2 & 2 & $\begin{array}{c}\text { Not Yet } \\
\text { Determined }\end{array}$ \\
\hline STAR & Communications & ATIS Arrival Frequency & 2 & 1 & 2 \\
\hline STAR & Communications & Communications Boundaries & 2 & 3 & $\begin{array}{c}\text { Not Yet } \\
\text { Determined }\end{array}$ \\
\hline STAR & Communications & Lost Comm Procedure & 2 & 2,3 & 3 \\
\hline STAR & Communications & Lost Comm Procedure Outline Lines & 2 & 3 & 2 \\
\hline STAR & Communications & Transponder Setting where appropriate & 2 & 2 & 2 \\
\hline STAR & Course Definition & Heading & 1 & 1 & 1 \\
\hline STAR & Course Definition & MEA/MOCA & 2 & 3 & 1 \\
\hline STAR & Course Definition & Radial & 1 & 1 & 1 \\
\hline STAR & Course Definition & Segment Mileages & 1 & $1,2,3$ & 1 \\
\hline STAR & Course Definition & Track & 1 & 1 & 1 \\
\hline STAR & Course Definition & VOR Change Over Points & 2 & - & $\begin{array}{c}\text { Not Yet } \\
\text { Determined }\end{array}$ \\
\hline STAR & Geography & Contour Interval Legend & 2 & 2,4 & 3 \\
\hline
\end{tabular}




\begin{tabular}{|c|c|c|c|c|c|}
\hline $\begin{array}{l}\text { Chart } \\
\text { Type }\end{array}$ & $\begin{array}{l}\text { Information } \\
\text { Category }\end{array}$ & Information Element & $\begin{array}{c}\text { SAE ARP } \\
5621 \\
(2011)\end{array}$ & $\begin{array}{c}\text { Pepitone } \\
\text { et al. } \\
(2014)\end{array}$ & $\begin{array}{c}\text { Current } \\
\text { Survey }\end{array}$ \\
\hline STAR & Geography & Cultural Features & 3 & - & $\begin{array}{c}\text { Not Yet } \\
\text { Determined }\end{array}$ \\
\hline STAR & Geography & $\begin{array}{l}\text { Highest Reference Point (within neat } \\
\text { lines) }\end{array}$ & 2 & - & 1 \\
\hline STAR & Geography & $\begin{array}{l}\text { International Boundaries (higher } \\
\text { criticality where appropriate) }\end{array}$ & 2 & - & $\begin{array}{c}\text { Not Yet } \\
\text { Determined }\end{array}$ \\
\hline STAR & Geography & $\begin{array}{l}\text { Neat Lines (i.e., the lines which separate } \\
\text { the chart from the margins) }\end{array}$ & 3 & - & $\begin{array}{c}\text { Not Yet } \\
\text { Determined }\end{array}$ \\
\hline STAR & Geography & Parallels and Meridians & 3 & - & 3 \\
\hline STAR & Geography & $\begin{array}{l}\text { Parallels and Meridians with AMAs, } \\
\text { OROCAs, MORAs }\end{array}$ & 2 & - & 3 \\
\hline STAR & Geography & Range & - & 3 & 1 \\
\hline STAR & Geography & Spot Elevations & 2 & - & 1 \\
\hline STAR & Geography & Terrain Contour Elevations & 2 & 2 & $\begin{array}{c}\text { Not Yet } \\
\text { Determined }\end{array}$ \\
\hline STAR & Geography & Terrain Contours & 2 & 2 & 1 \\
\hline STAR & Geography & Water Features & 3 & - & $\begin{array}{c}\text { Not Yet } \\
\text { Determined }\end{array}$ \\
\hline STAR & Holding Pattern & Holding Pattern Altitude & 1 & 3 & 1 \\
\hline STAR & Holding Pattern & Holding Pattern Courses & 2 & 1 & 1 \\
\hline STAR & Holding Pattern & Holding Pattern Depiction & 1 & 1 & 1 \\
\hline STAR & Holding Pattern & Holding Pattern Leg Length & 2 & 3 & 1 \\
\hline STAR & Holding Pattern & Holding Pattern Speed & 2 & 2 & 2 \\
\hline STAR & Holding Pattern & Holding Pattern Time & 2 & 2,3 & 2 \\
\hline STAR & Identification & Airport ICAO Identifier & 1 & 1 & 1 \\
\hline STAR & Identification & Airport Name & 1 & 1 & $\begin{array}{c}\text { Not Yet } \\
\text { Determined }\end{array}$ \\
\hline STAR & Identification & Changes & 3 & - & 2 \\
\hline STAR & Identification & Chart Index Number/Page Number & 3 & - & 4 \\
\hline STAR & Identification & City/Location Name & 2 & 3 & 2 \\
\hline STAR & Identification & Effective Date & 3 & - & 2 \\
\hline STAR & Identification & $\begin{array}{l}\text { Procedure Identifier (e.g., } \\
\text { CNOG8.VNY) }\end{array}$ & 2 & 1 & 1 \\
\hline STAR & Identification & Procedure Name (e.g., Canoga Eight) & 1 & 1 & 1 \\
\hline STAR & Identification & Revision Date & 3 & - & 2 \\
\hline STAR & $\begin{array}{l}\text { Instrument Procedure } \\
\text { Courses/Tracks }\end{array}$ & Identifier (i.e., CNOG8.AVE) & 1 & 1 & 1 \\
\hline STAR & $\begin{array}{l}\text { Instrument Procedure } \\
\text { Courses/Tracks }\end{array}$ & Symbol (e.g., line style, etc.) & 1 & 1 & 1 \\
\hline STAR & $\begin{array}{l}\text { Intersection /Fixes on } \\
\text { Procedure }\end{array}$ & Identifier & 1 & 1 & 1 \\
\hline STAR & $\begin{array}{l}\text { Intersection /Fixes on } \\
\text { Procedure }\end{array}$ & Latitude/Longitudes & 3 & - & 3 \\
\hline STAR & $\begin{array}{l}\text { Intersection /Fixes on } \\
\text { Procedure }\end{array}$ & MRA & 3 & - & $\begin{array}{c}\text { Not Yet } \\
\text { Determined }\end{array}$ \\
\hline
\end{tabular}




\begin{tabular}{|c|c|c|c|c|c|}
\hline $\begin{array}{l}\text { Chart } \\
\text { Type }\end{array}$ & $\begin{array}{l}\text { Information } \\
\text { Category }\end{array}$ & Information Element & $\begin{array}{c}\text { SAE ARP } \\
5621 \\
(2011) \\
\end{array}$ & $\begin{array}{c}\text { Pepitone } \\
\text { et al. } \\
\text { (2014) }\end{array}$ & $\begin{array}{l}\text { Current } \\
\text { Survey }\end{array}$ \\
\hline STAR & $\begin{array}{l}\text { Intersection /Fixes on } \\
\text { Procedure }\end{array}$ & Names & 2 & 1 & 1 \\
\hline STAR & $\begin{array}{l}\text { Intersection /Fixes on } \\
\text { Procedure }\end{array}$ & Symbol & 1 & 2 & 1 \\
\hline STAR & $\begin{array}{l}\text { Minimum Area/Sector } \\
\text { Altitudes }\end{array}$ & $\begin{array}{l}\text { AMA, OROCA, or grid MORA where } \\
\text { established }\end{array}$ & 2 & 3 & $\begin{array}{c}\text { Not Yet } \\
\text { Determined }\end{array}$ \\
\hline STAR & $\begin{array}{l}\text { Minimum Area/Sector } \\
\text { Altitudes }\end{array}$ & Minimum Radar Altitudes and Sectors & 3 & - & $\begin{array}{c}\text { Not Yet } \\
\text { Determined }\end{array}$ \\
\hline STAR & $\begin{array}{l}\text { Minimum Area/Sector } \\
\text { Altitudes }\end{array}$ & MSA Distance when other than 25nm & 2 & 2 & $\begin{array}{c}\text { Not Yet } \\
\text { Determined }\end{array}$ \\
\hline STAR & $\begin{array}{l}\text { Minimum Area/Sector } \\
\text { Altitudes }\end{array}$ & MSA Minimum Altitudes & 2 & - & 1 \\
\hline STAR & $\begin{array}{l}\text { Minimum Area/Sector } \\
\text { Altitudes }\end{array}$ & MSA Reference Point/Center & 2 & 2 & $\begin{array}{c}\text { Not Yet } \\
\text { Determined }\end{array}$ \\
\hline STAR & $\begin{array}{l}\text { Minimum Area/Sector } \\
\text { Altitudes }\end{array}$ & MSA Sector Radials & 2 & - & $\begin{array}{c}\text { Not Yet } \\
\text { Determined }\end{array}$ \\
\hline STAR & $\begin{array}{l}\text { Navaid Used to Form } \\
\text { Fixes }\end{array}$ & DME Availability (Text information) & 3 & - & 2 \\
\hline STAR & $\begin{array}{l}\text { Navaid Used to Form } \\
\text { Fixes }\end{array}$ & DME Distances that form fixes & 2 & 1 & 1 \\
\hline STAR & $\begin{array}{l}\text { Navaid Used to Form } \\
\text { Fixes }\end{array}$ & Navaid Class & 3 & - & 3 \\
\hline STAR & $\begin{array}{l}\text { Navaid Used to Form } \\
\text { Fixes }\end{array}$ & Navaid Frequency/Channel & 2 & 1,2 & 1 \\
\hline STAR & $\begin{array}{l}\text { Navaid Used to Form } \\
\text { Fixes }\end{array}$ & Navaid Identifier & 1 & 2 & 1 \\
\hline STAR & $\begin{array}{l}\text { Navaid Used to Form } \\
\text { Fixes }\end{array}$ & Navaid Latitude/Longitude & 3 & - & 3 \\
\hline STAR & $\begin{array}{l}\text { Navaid Used to Form } \\
\text { Fixes }\end{array}$ & Navaid Morse Code & 2 & 3 & $\begin{array}{c}\text { Not Yet } \\
\text { Determined }\end{array}$ \\
\hline STAR & $\begin{array}{l}\text { Navaid Used to Form } \\
\text { Fixes }\end{array}$ & Navaid Name & 2 & 1 & 1 \\
\hline STAR & $\begin{array}{l}\text { Navaid Used to Form } \\
\text { Fixes }\end{array}$ & Navaid Radials/Bearings that form fixes & 2 & 3 & 1 \\
\hline STAR & $\begin{array}{l}\text { Navaid Used to Form } \\
\text { Fixes }\end{array}$ & Navaid Symbol & 1 & 1,2 & 1 \\
\hline STAR & $\begin{array}{l}\text { Navaid Used to Form Leg } \\
\text { of Procedure }\end{array}$ & DME Availability (Text information) & 3 & - & 2 \\
\hline STAR & $\begin{array}{l}\text { Navaid Used to Form Leg } \\
\text { of Procedure }\end{array}$ & Navaid Class & 3 & - & 3 \\
\hline STAR & $\begin{array}{l}\text { Navaid Used to Form Leg } \\
\text { of Procedure }\end{array}$ & Navaid Frequency/Channel & 2 & 1 & 1 \\
\hline STAR & $\begin{array}{l}\text { Navaid Used to Form Leg } \\
\text { of Procedure }\end{array}$ & Navaid Identifier & 1 & 2 & 1 \\
\hline STAR & $\begin{array}{l}\text { Navaid Used to Form Leg } \\
\text { of Procedure }\end{array}$ & Navaid Latitude/Longitude & 3 & - & 3 \\
\hline STAR & $\begin{array}{l}\text { Navaid Used to Form Leg } \\
\text { of Procedure }\end{array}$ & Navaid Morse Code & 2 & 3 & 2 \\
\hline STAR & $\begin{array}{l}\text { Navaid Used to Form Leg } \\
\text { of Procedure }\end{array}$ & Navaid Name & 2 & 2 & 1 \\
\hline
\end{tabular}




\begin{tabular}{|c|c|c|c|c|c|}
\hline $\begin{array}{l}\text { Chart } \\
\text { Type }\end{array}$ & $\begin{array}{l}\text { Information } \\
\text { Category }\end{array}$ & Information Element & $\begin{array}{c}\text { SAE ARP } \\
5621 \\
(2011) \\
\end{array}$ & $\begin{array}{c}\text { Pepitone } \\
\text { et al. } \\
\text { (2014) }\end{array}$ & $\begin{array}{l}\text { Current } \\
\text { Survey }\end{array}$ \\
\hline STAR & $\begin{array}{l}\text { Navaid Used to Form Leg } \\
\text { of Procedure }\end{array}$ & Navaid Symbol & 1 & 2 & 1 \\
\hline STAR & Navigation & FIR/UIR Boundaries & 3 & - & $\begin{array}{c}\text { Not Yet } \\
\text { Determined }\end{array}$ \\
\hline STAR & Navigation & $\begin{array}{l}\text { Prohibited, Restricted and Danger } \\
\text { Airspace Graphic }\end{array}$ & 1 & 3 & 1 \\
\hline STAR & Navigation & $\begin{array}{l}\text { Prohibited, Restricted and Danger } \\
\text { Airspace Label }\end{array}$ & 3 & - & $\begin{array}{c}\text { Not Yet } \\
\text { Determined }\end{array}$ \\
\hline STAR & Navigation & $\begin{array}{l}\text { Prohibited, Restricted and Danger } \\
\text { Airspace Narrative }\end{array}$ & 3 & - & 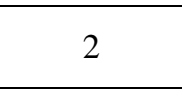 \\
\hline STAR & Navigation & Special Use Airspace - Other & 2 & 2 & 2 \\
\hline STAR & Navigation & Transition Level & 2 & 2 & 2 \\
\hline STAR & Obstacle & Obstacle Symbols and Elevation & 3 & - & 1 \\
\hline STAR & Textual Information & Crossing Altitude Restrictions & 1 & 1 & 1 \\
\hline STAR & Textual Information & General Notes & 2 & 4 & 2 \\
\hline STAR & Textual Information & Noise Abatement & 2 & 3 & 2 \\
\hline STAR & Textual Information & Notes & 2 & 4 & 2 \\
\hline STAR & Textual Information & $\begin{array}{l}\text { Performance limitations (e.g., bank } \\
\text { limits) }\end{array}$ & 2 & 3 & 2 \\
\hline STAR & Textual Information & Procedural Data Notes & 2 & 3,4 & 2 \\
\hline STAR & Textual Information & Runway arrival text & 1 & 2 & 2 \\
\hline STAR & Textual Information & Speed restrictions & 1 & 1,2 & 1 \\
\hline STAR & Textual Information & Text-Only Procedures & 1 & 4 & 2 \\
\hline STAR & Textual Information & Transition Text & 3 & - & 2 \\
\hline STAR & Transitions & Transition Course - Magnetic Values & 1 & 1 & $\begin{array}{c}\text { Not Yet } \\
\text { Determined }\end{array}$ \\
\hline STAR & Transitions & $\begin{array}{l}\text { Transition Course notes (e.g., DME } \\
\text { required) }\end{array}$ & 2 & 3 & 2 \\
\hline STAR & Transitions & Transition Courses - MEAs, MOCAs & 2 & 3 & $\begin{array}{c}\text { Not Yet } \\
\text { Determined }\end{array}$ \\
\hline STAR & Transitions & Transition Courses - segment mileages & 2 & 1 & $\begin{array}{c}\text { Not Yet } \\
\text { Determined }\end{array}$ \\
\hline STAR & Transitions & Transition Courses computer codes & 2 & 3 & 2 \\
\hline STAR & Transitions & Transition Courses Depiction & 1 & 1 & 1 \\
\hline STAR & Transitions & Transition Name & 1 & 1 & 1 \\
\hline STAR & Transitions & Transition Text & 1 & 3,4 & 2 \\
\hline
\end{tabular}

\section{Discussion}

The purpose of this study was to further examine the criticality ratings provided in SAE ARP 5621 (SAE International, 2011) to try to identify minimum information requirements for electronic data-driven charts. This study focused on four different chart types and included pilots 
from four different types of operations. We made several attempts to classify the information elements identified as “not yet determined.” In reviewing the chi-square analyses, we noticed that there were differences in the pilot populations classifying the information element - for example, an information element that was identified as level 1 (displayed at all times) for air transport and military pilots may have been identified as level 2 (displayed initially; can be toggled off/on) by general aviation and commuter pilots. We did not pursue these individual differences, however, because we believed that the design of aeronautical charts needed to be achieved in a pilot-agnostic fashion. That is, we should not be defining one minimum set of information elements for air transport operations and another set for general aviation operations, as the same pilot could fly both types of operations and the lack of consistency in the same aeronautical charting application could induce error.

We compared the results of our survey to those ratings in SAE ARP 5621 (SAE International, 2011) and in Pepitone et al. (2014). We conducted two comparisons. The first examined the number of information elements with the same rating across all three sources - our survey, SAE ARP 5621, and Pepitone et al. Because Pepitone et al. did not address IFR/Enroute charts in their study, we did not make this comparison for that chart type. In examining the ratings across all the aeronautical chart types addressed in our study, the ratings matched across all three sources for 33\% of the information elements. A breakdown by chart type is shown in Table 8. The second column of Table 8 shows the number of information elements included in the comparisons for each aeronautical chart type, and the third column shows the number of information elements matching across all three sources. We excluded all 63 information elements identified as “Not Yet Determined” from this comparison, because we could not classify those information elements.

The second comparison examined the agreement between our ratings and either SAE ARP 5261 (SAE International, 2011) or Pepitone et al. (2014). A comparison of the ratings across all aeronautical chart types identified that $40 \%$ of the information element ratings matched one of the two sources (see the fourth column of Table 8). 
Table 8

Comparison of Information Element Ratings: Results

\begin{tabular}{|c|c|c|c|c|c|}
\hline Chart Type & $\begin{array}{c}\text { Total } \\
\text { Number of } \\
\text { Information } \\
\text { Elements }\end{array}$ & $\begin{array}{l}\text { Number of } \\
\text { Information } \\
\text { Elements } \\
\text { included in } \\
\text { Analysis* }\end{array}$ & $\begin{array}{l}\text { All Sources } \\
\text { in } \\
\text { Agreement }\end{array}$ & $\begin{array}{l}\text { Agreement with } \\
\text { SAE ARP } 5261 \\
\text { (2011) OR } \\
\text { Pepitone et al. } \\
\text { (2014) }\end{array}$ & $\begin{array}{c}\text { Total } \\
\text { Matching } \\
\text { One Source } \\
\text { or More }\end{array}$ \\
\hline $\begin{array}{l}\text { Instrument } \\
\text { Approach } \\
\text { Procedure (IAP) }\end{array}$ & 131 & 117 & $51(44 \%)$ & $29(25 \%)$ & $80(68 \%)$ \\
\hline $\begin{array}{l}\text { Standard } \\
\text { Instrument } \\
\text { Departure (SID) }\end{array}$ & 103 & 86 & 20 (23\%) & 39 (45\%) & 59 (69\%) \\
\hline $\begin{array}{l}\text { Standard } \\
\text { Terminal } \\
\text { Arrival Route } \\
\text { (STAR) }\end{array}$ & 103 & 81 & 22 (27\%) & 35 (43\%) & 57 (70\%) \\
\hline Enroute (IFR) & 90 & 80 & N/A*** & 43 (54\%) & 43 (54\%) \\
\hline TOTAL & 427 & 364 & $93(33 \%)^{*}$ & $146(40 \%)$ & 239 (66\%) \\
\hline
\end{tabular}

In total, ratings for approximately 66\% of information elements matched the ratings in SAE ARP 5261 (SAE International, 2011) and/or Pepitone et al. (2014), leaving 34\% of information elements for which there was no match.

As one way to describe the results, we developed prototype charts based on the determined level of importance for each information element to show a pictographic representation of the findings. We also wanted to see if any of the "Not Yet Determined"-information elements could be classified during the prototype chart development process due to its relationship with other information elements. The levels of importance for the information elements were distinguished by color to show which information elements could be added/removed as required. Figure 5 presents examples of each layer for a STAR chart. Figure 5(a) shows a cross-section of the STAR for Bellingham, Washington; this image depicts all the information shown on that cross- 
section of the aeronautical chart. Figure 5 (b) shows the information elements identified as level 1 (displayed at all times). A comparison of Figure 5 (a) to (b) shows that procedure notes on the right side of the chart and the arrival route description at the bottom of the chart were removed.

Figure 5 (c) shows a combination of the level 1 and level 2 information elements; these are the information elements that need to be displayed at all times and those displayed initially but that can be toggled on/off. Finally, Figure 5 (d) shows the level 1 and level 3 information elements (information elements that do not need to be presented initially and can be manually selected).

Figure 6, Figure 7, and Figure 8 are examples of other chart types (IFR, SID, IAP, respectively). Image (a) within each Figure 6-8 chart example represents a cross-section of that particular chart type and shows all the information elements within that cross-section. Image (b) of each figure shows information elements identified as Level 1 (displayed at all times). Image (c) shows information elements identified as Level 1 (displayed at all time) and Level 2 (displayed initially, but can be toggled off/on). Image (d), when applicable, shows information elements identified as Level 1 (displayed at all times) and Level 3 (not displayed initially, but can be toggled on/off). For the IAP (Figure 7) and SID (Figure 8) example charts, there were no Level 3 information elements depicted on that particular cross-section of the chart.

The prototype charts stimulated discussions about whether additional information within a level could be decluttered - e.g., information that was classified as Level 1 (Displayed at all times) but that may not be relevant to the actual procedure being flown. Additionally, we wanted to consider whether these depictions were consistent with other concepts being proposed for electronic aeronautical charts. There are many approaches for decluttering that may be considered by criticality: decluttering by route, by aircraft equipage, time, phase of flight, etc. Each of these approaches introduces different considerations, but in general, there should be a way to convey to the flightcrew or pilot that the status of the decluttering, and a means must be provided for the pilot or flightcrew to recall/retrieve the information that was decluttered. 


\section{Figure 5}

Mock-up example of Standard Terminal Arrival Route (STAR) Chart. (a) Full chart, (b) Information elements that are displayed at all times (Level 1 only), (c) information elements displayed at all times (Level 1) and those displayed initially but that can be toggled on/off (Level 2), and (d) information elements displayed at all times (Level 1) and those that don't need to be presented initially and can be manually selected (Level 3).

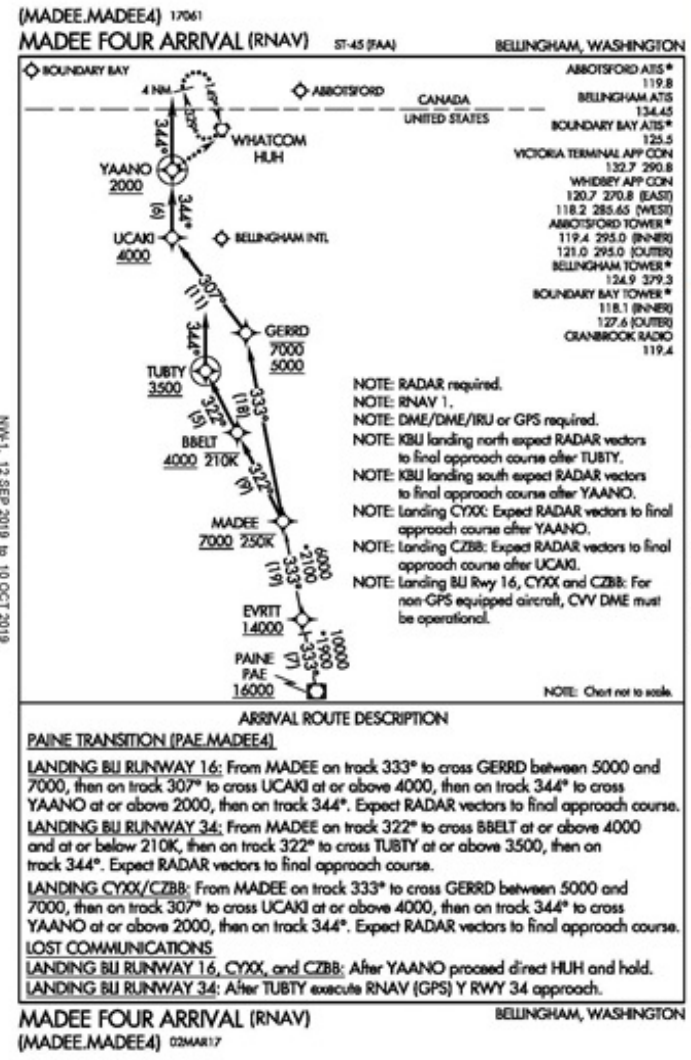

(a) Full Chart

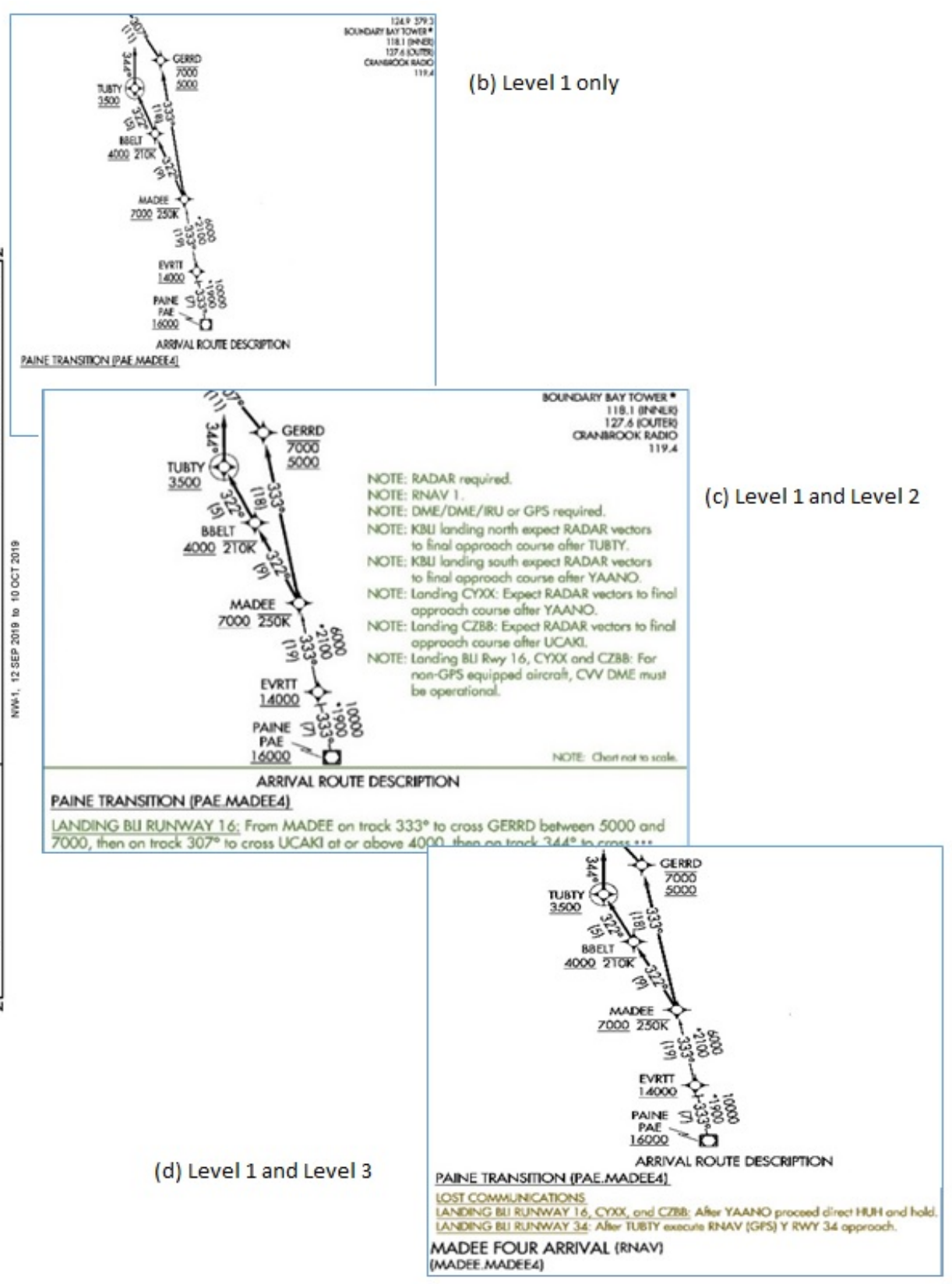




\section{Figure 6}

Mock-up example of Enroute Instrument Flight Rule (IFR) Chart. (a) Full chart, (b) Information elements that are displayed at all times (Level 1 only), (c) information elements displayed at all times (Level 1) and those displayed initially but that can be toggled on/off (Level 2), and (d) information elements displayed at all times (Level 1) and those that don't need to be presented initially and can be manually selected (Level 3).

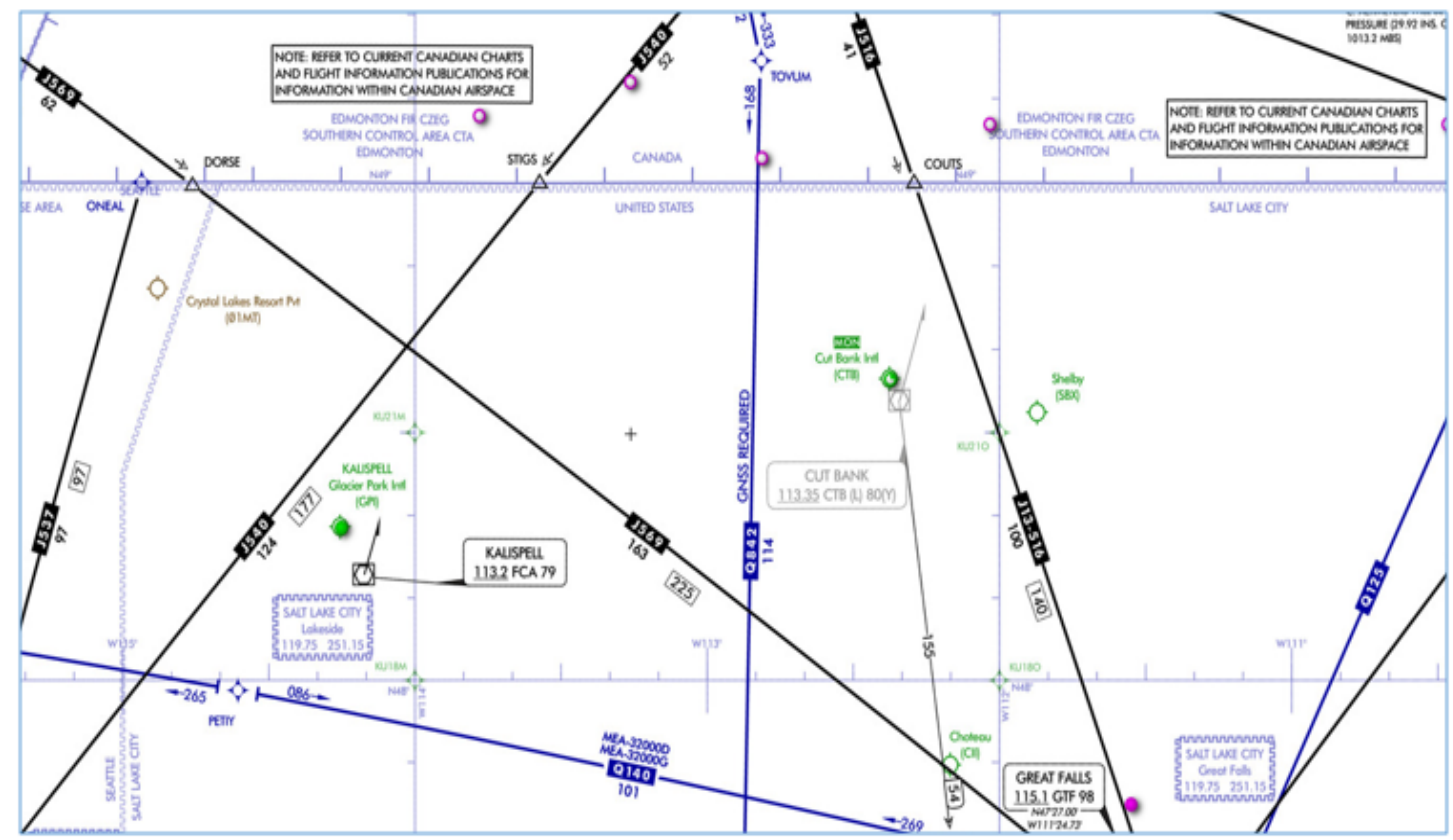

(a) Full Chart

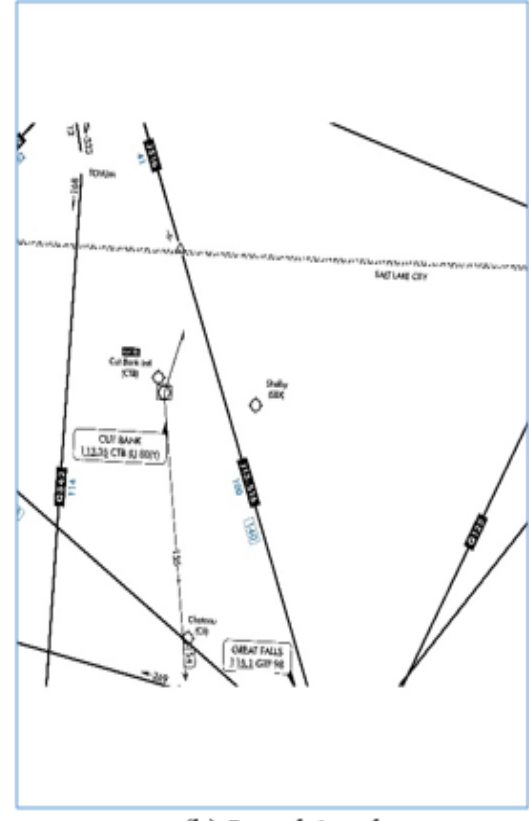

(b) Level 1 only

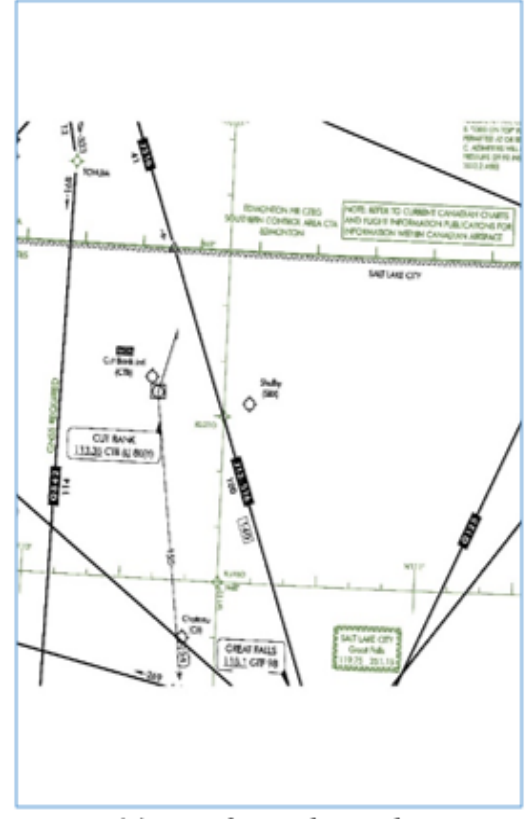

(c) Level 1 and Level 2

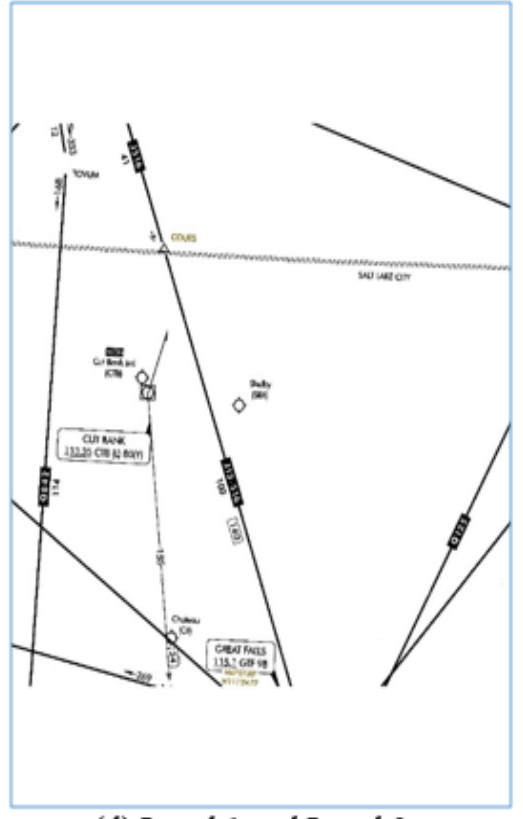

(d) Level 1 and Level 3 


\section{Figure 7}

Mock-up example of Standard Instrument Departure (SID) Chart. (a) Full chart, (b) Information elements that are displayed at all times (Level 1 only), and (c) information elements displayed at all times (Level 1) and those displayed initially but that can be toggled on/off (Level 2). There were no Level 3 information elements displayed on this specific procedure.

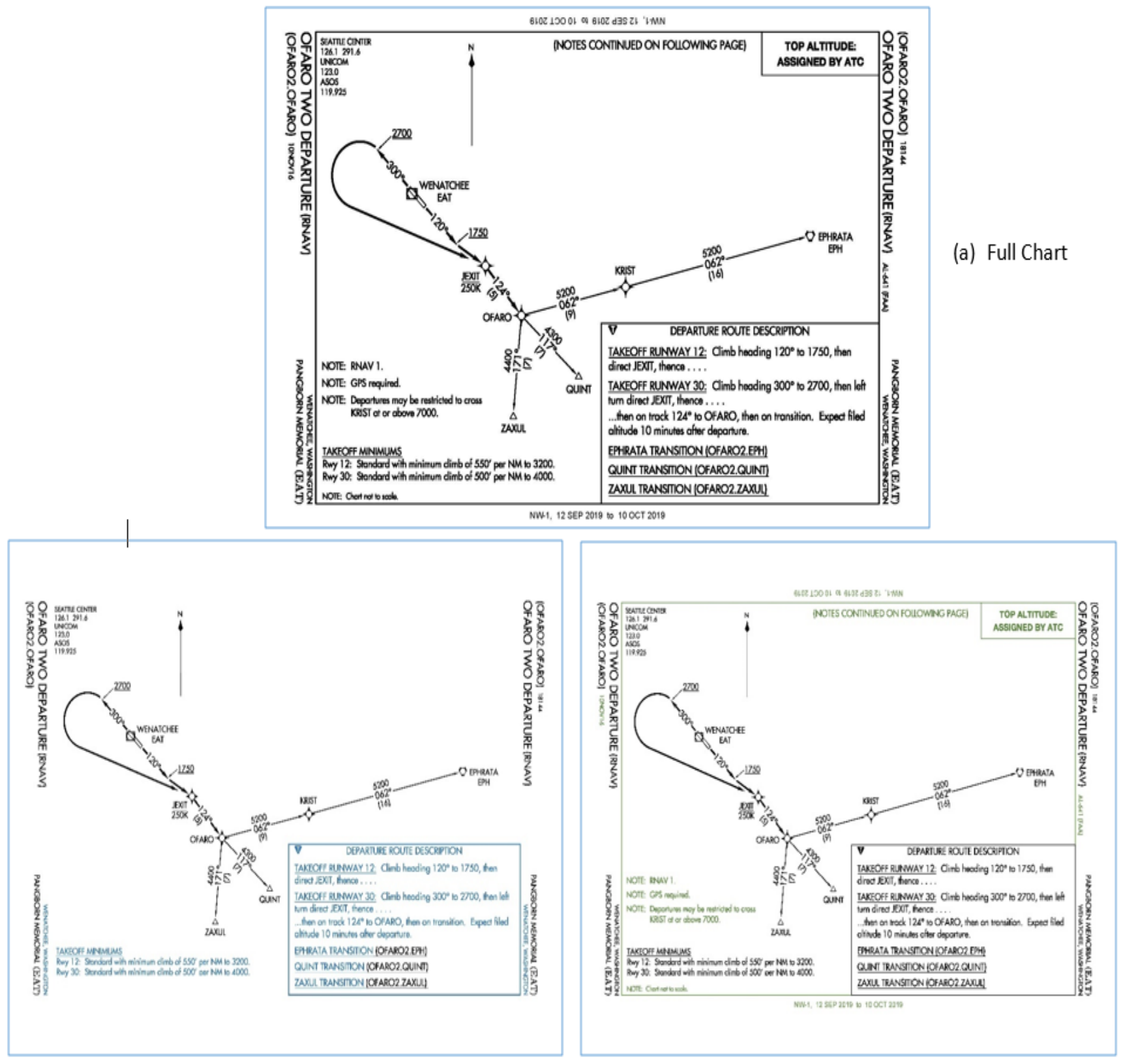

(b) Level 1 Only

(b) Level 1 and Level 2 


\section{Figure 8}

Mock-up example of Instrument Approach Procedure (IAP) Chart. (a) Full chart, (b) Information elements that are displayed at all times (Level 1 only), and (c) information elements displayed at all times (Level 1) and those displayed initially but that can be toggled on off (Level 2). There were no Level 3 information elements displayed on this specific procedure.

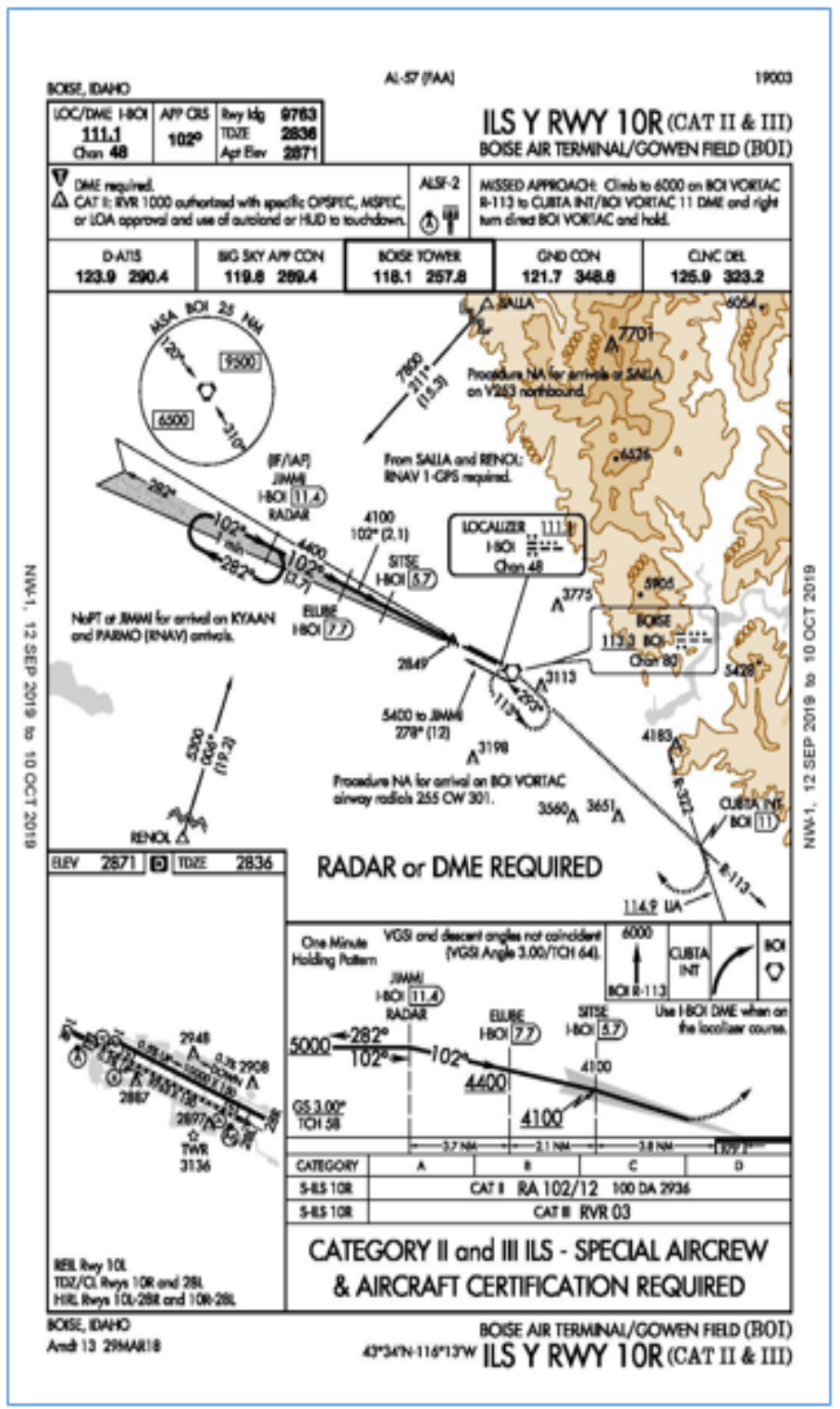

(a) Full Chart

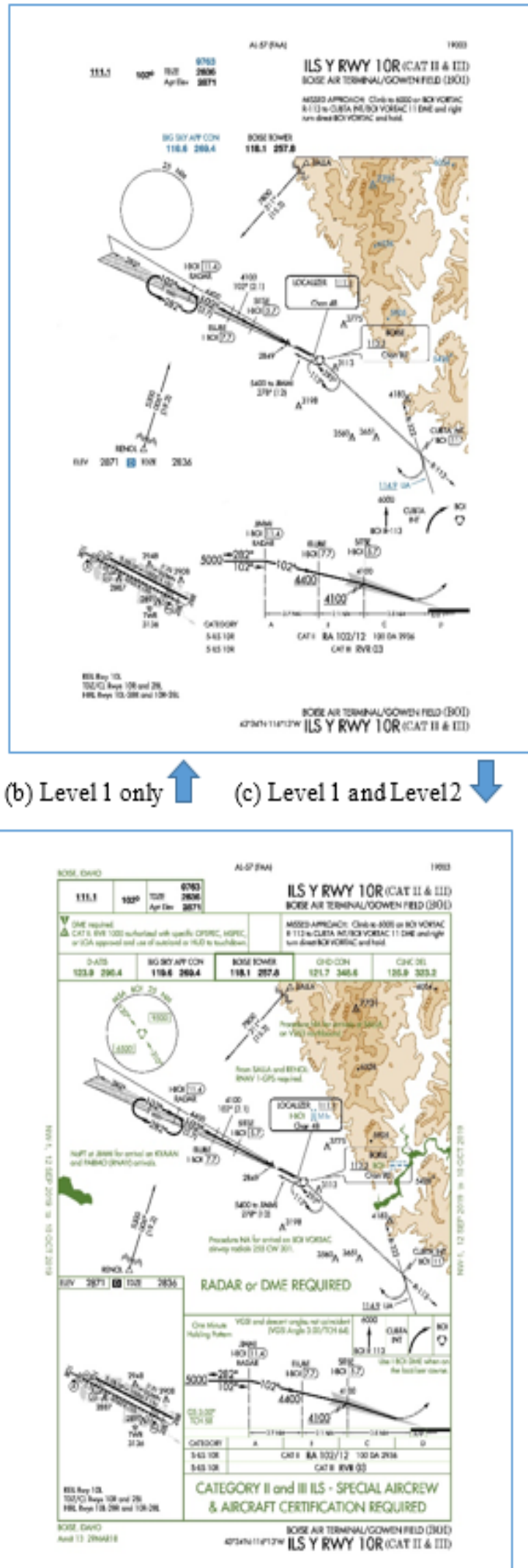




\section{Conclusions}

The purpose of this study was to identify a set of minimum information elements for userconfigurable electronic aeronautical charts. The results are only a first step in identifying critical information elements for configurable electronic charts. The methodology for this study used a survey framework, but additional research is needed to validate the survey findings due to the following limitations. First, the results only reflect pilot opinions; we have not had pilots "fly" with the prototype charts yet. Second, there was no common definition for each information element across end users, and the sample charts we provided did not contain all the information elements. Third, the criticality of an information element may vary depending on the manufacturer's intended function. This research focused on scenarios in which pilots brief with a fixed chart and fly with a reconfigurable electronic chart, but they always have access to the fixed chart. As flight deck systems become more integrated, it is conceivable that the charting application may be combined with a moving map-type avionics system as a replacement for the aeronautical chart. Such a configuration would require a re-evaluation of the criticality of information elements as well as other human factors considerations. 


\section{Acknowledgments}

The research was conducted under the Flight Deck Program Directive/Level of Effort Agreement between the FAA NextGen Human Factors Division (ANG-C1) and the Aerospace Human Factors Research Division (AAM-500) of the FAA Civil Aerospace Medical Institute. The authors would like to thank our program manager, Katrina Avers. We would also like to thank Dan Jack, Shijing Liu, Inchul Choi, and Suzanne Thomas of Cherokee Nation Businesses; and Danielle Hiltunen of the US DOT Volpe Center for their contributions. Finally, we would like to thank all the pilots who participated. 


\section{References}

Blomberg, R. D., Bishop, E. W., \& Hamilton, J. W. (1995). Evaluation of prototype air carrier instrument approach procedure charts (Report No. DOT/FAA/95/44, DOT-VNTSC-FAA95-24). US Department of Transportation, Federal Aviation Administration. Available at: https://rosap.ntl.bts.gov/view/dot/8782

Butchibabu, A., \& Hansman, R. J. (2012). Evaluating the depiction of complex RNAV/RNP Procedures and analyzing a potential de-cluttering technique (Unpublished master's thesis). Massachusetts Institute of Technology, Cambridge.

Hansman, R., \& Mykityshyn, M. (1995a). An exploratory survey of information requirements for instrument approach charts (Report No. DOT/FAA/AAR-95/2). US Department of Transportation, Federal Aviation Administration. Available at: https://rosap.ntl.bts.gov/view/dot/8642

Hansman, R. J., and Mykityshyn, M. (1995b). Current issues in the design and information content of instrument approach charts (Report No. DOT/FAA/ARR-95/1). US Department of Transportation, Federal Aviation Administration. Available at: http://www.dtic.mil/get-trdoc/pdf?AD=ADA294752

Hofer, E. F., Palen, L.A., \& Possolo, A. (1993). Flight deck information management: An experimental study of functional integration of approach data. Proceedings of the 7th International Symposium on Aviation Psychology. Columbus, $\mathrm{OH}$ : Department of Aviation, Ohio State University.

Moacdieh, N., \& Sarter, N. (2015). Data density and poor organization: analyzing the performance and Attentional effects of two aspects of display clutter. Proceedings of the Human Factors and Ergonomics Society Annual Meeting, 59(1), 1336-

1340. https://doi.org/10.1177/1541931215591221

Multer, J., Warner, M., Disario, R., \& Huntley, M. (1991). Design considerations for IAP charts: Approach course track and communication frequencies (Report No. DOT/FAA/RD-91/19). US Department of Transportation, Federal Aviation Administration. Available at: http://rosap.ntl.bts.gov/view/dot/8699

Osborne, D. W., \& Huntley, M. S. (1992). Design of instrument approach procedure charts: Comprehension speed of missed approach instructions coded in text or icons (Report No. DOT/FAA/RD-92/3). US Department of Transportation, Federal Aviation Administration. Available at: https://rosap.ntl.bts.gov/view/dot/8637

Osborne, D. W., Huntley, M. S. Jr., Turner, J. W., \& Donovan, C. M. (1995). The effect of instrument approach procedure chart design on pilot search speed and response accuracy: Flight test results (Report No. DOT/FAA/AR-95/8, DOT-VNTSCS-FAA-95-13). US 
Department of Transportation, Federal Aviation Administration. Available at: https://rosap.ntl.bts.gov/view/dot/8650

Pepitone, D., Ball, J., \& Letsu-Dake, E. (2014). NextGen Flight Deck information displays: Recommendations for managing display complexity with electronic chart information (Version 2). Deer Valley, AZ: Honeywell. Available at: http://www.hf.faa.gov/FAAAJP61/Honeywell\%20Report\%20on\%20Electronic\%20Charts\% 20Feb\%202014\%20Rev\%202.0.pdf

SAE International. (2011). Electronic Display of Aeronautical Information (Aerospace Recommended Practice No. 5621). https://www.sae.org/standards/content/arp5621/

Schvaneveldt, R. W., Beringer, D. B., \& Lamonica, J. A. (2001). Priority and organization of information accessed by pilots in various phases of flight. The International Journal of Aviation Psychology, 11(3), 253-280. https://doi.org/10.1207/S15327108IJAP1103_02

Stewart, M., Laraway, S., Jordan, K., \& Feary, M. S. (2016). Simplified approach charts improve data retrieval performance. Journal of Cognitive Engineering and Decision Making, 11(2), 166-183. https://doi.org/10.1177\%2F1555343416675556

Teichner, W. H., \& Mocharnuk, J. B. (1979). Visual search for complex targets. Human Factors, 21(3), 259-275. https://doi.org/10.1177/001872087902100302

Treisman, A. (1988). Features and Objects: The Fourteenth Bartlett Memorial Lecture. The Quarterly Journal of Experimental Psychology. 40A(2), 201-237. https://doi.org/10.1080/02724988843000104

Treisman, A. M., \& Gelade, G. (1980). A feature-integration theory of attention. Cognitive Psychology, 12(1), 97-136. https://doi.org/10.1016/0010-0285(80)90005-5

Yantis, S. (1993). Stimulus-driven attentional capture. Current Directions in Psychological Science, 2(5), 156-161. https://doi.org/10.1111/1467-8721.ep10768973

Yeh, M., \& Chandra, D. C. (2003). Air transport pilots' information priorities for surface moving map displays. Proceedings of the Human Factors and Ergonomics Society 47th Annual Meeting. Santa Monica, CA: Human Factors and Ergonomics Society. 


\section{Appendix A}

\section{Chi-Square Results}

Summary: Below is the table of chi-square results for individual information elements. Q1, Q2, and Q3 correspond to the questions in the data analysis section and are addressed by the Yes/No in each cell. An asterisk (*) indicates that the results of the chi-square analysis was significant. We performed the Bonferroni correction on a subset of the information elements and found that the significance of the results were unchanged. We do not report those numbers here.

\begin{tabular}{|c|c|c|c|c|c|c|}
\hline $\begin{array}{l}\text { Chart } \\
\text { Type }\end{array}$ & $\begin{array}{l}\text { Information } \\
\text { Category }\end{array}$ & Information Element & $\begin{array}{l}\text { Q1: Should it be } \\
\text { displayed to } \\
\text { execute } \\
\text { procedure? }\end{array}$ & $\begin{array}{l}\text { Q2: Displayed } \\
\text { at all times? }\end{array}$ & $\begin{array}{l}\text { Q3: Displayed } \\
\text { Initially? }\end{array}$ & $\begin{array}{l}\text { Importance } \\
\text { Level }\end{array}$ \\
\hline IAP & Identification & Revision Date & $\begin{array}{c}\text { Yes } \\
x^{2}(1, \mathrm{~N}=106)= \\
29.58^{*}\end{array}$ & $\begin{array}{c}\text { No } \\
x^{2}(1, \mathrm{~N}=81) \\
=62.23^{*}\end{array}$ & $\begin{array}{c}\text { Yes } \\
x^{2}(1, \mathrm{~N}=76) \\
=10.32^{*}\end{array}$ & 2 \\
\hline IAP & Identification & $\begin{array}{l}\text { Chart Index } \\
\text { Number/Page Number }\end{array}$ & $\begin{array}{c}\text { Yes } \\
x^{2}(1, \mathrm{~N}=106)= \\
8.49 *\end{array}$ & $\begin{array}{c}\text { No } \\
x^{2}(1, \mathrm{~N}=68) \\
=46.12^{*}\end{array}$ & $\begin{array}{c}\text { Yes } \\
x^{2}(1, \mathrm{~N}=62) \\
=4.13^{*}\end{array}$ & 2 \\
\hline IAP & Identification & Effective Date & $\begin{array}{c}\text { Yes } \\
x^{2}(1, \mathrm{~N}=106)= \\
43.62^{*}\end{array}$ & $\begin{array}{c}\text { No } \\
x^{2}(1, \mathrm{~N}=87) \\
=57.94^{*}\end{array}$ & $\begin{array}{c}\text { Yes } \\
x^{2}(1, N=79) \\
=15.51^{*}\end{array}$ & 2 \\
\hline IAP & Identification & City/Location Name & $\begin{array}{c}\text { Yes } \\
x^{2}(1, \mathrm{~N}=106)= \\
73.06^{*}\end{array}$ & $\begin{array}{c}\text { No } \\
x^{2}(1, \mathrm{~N}=97) \\
=12.63^{*}\end{array}$ & $\begin{array}{c}\text { Yes } \\
x^{2}(1, \mathrm{~N}=66) \\
=37.88^{*}\end{array}$ & 2 \\
\hline IAP & Identification & Airport Name & $\begin{array}{c}\text { Yes } \\
x^{2}(1, \mathrm{~N}=106)= \\
83.36^{*} \\
\end{array}$ & $\begin{array}{c}\text { No preference } \\
x^{2}(1, \mathrm{~N}= \\
100)=0.16 \\
\end{array}$ & & 1 \\
\hline IAP & Identification & Airport ICAO Identifier & $\begin{array}{c}\text { Yes } \\
x^{2}(1, \mathrm{~N}=104)= \\
88.62^{*}\end{array}$ & $\begin{array}{c}\text { Yes } \\
x^{2}(1, \mathrm{~N}= \\
100)=4.84^{*}\end{array}$ & & 1 \\
\hline IAP & Identification & Procedure Name & $\begin{array}{c}\text { Yes } \\
x^{2}(1, \mathrm{~N}=106)= \\
102.04^{*}\end{array}$ & $\begin{array}{c}\text { Yes } \\
x^{2}(1, \mathrm{~N}= \\
105)=30.94^{*}\end{array}$ & & 1 \\
\hline IAP & Identification & Airport Elevation & $\begin{array}{c}\text { Yes } \\
x^{2}(1, \mathrm{~N}=106)= \\
102.04^{*}\end{array}$ & $\begin{array}{c}\text { Yes } \\
x^{2}(1, \mathrm{~N}= \\
105)=26.75^{*}\end{array}$ & & 1 \\
\hline IAP & Identification & Changes & $\begin{array}{c}\text { Yes } \\
x^{2}(1, \mathrm{~N}=94)= \\
28.77^{*}\end{array}$ & $\begin{array}{c}\text { No } \\
x^{2}(1, N=73) \\
=30.26^{*}\end{array}$ & $\begin{array}{c}\text { Yes } \\
x^{2}(1, \mathrm{~N}=60) \\
=6.67^{*}\end{array}$ & 1 \\
\hline IAP & Geography & $\begin{array}{l}\text { Neat Lines (i.e., the lines } \\
\text { which separate the chart } \\
\text { from the margins) }\end{array}$ & $\begin{array}{c}\text { Yes } \\
x^{2}(1, \mathrm{~N}=101)= \\
36.84^{*}\end{array}$ & $\begin{array}{l}\text { No preference } \\
x^{2}(1, \mathrm{~N}=81) \\
=1.00\end{array}$ & & 2 \\
\hline
\end{tabular}




\begin{tabular}{|c|c|c|c|c|c|c|}
\hline $\begin{array}{l}\text { Chart } \\
\text { Type }\end{array}$ & $\begin{array}{l}\text { Information } \\
\text { Category }\end{array}$ & Information Element & $\begin{array}{l}\text { Q1: Should it be } \\
\text { displayed to } \\
\text { execute } \\
\text { procedure? }\end{array}$ & $\begin{array}{l}\text { Q2: Displayed } \\
\text { at all times? }\end{array}$ & $\begin{array}{l}\text { Q3: Displayed } \\
\text { Initially? }\end{array}$ & $\begin{array}{l}\text { Importance } \\
\text { Level }\end{array}$ \\
\hline IAP & Geography & Magnetic Variation & $\begin{array}{c}\text { Yes } \\
x^{2}(1, \mathrm{~N}=106)= \\
23.58^{*}\end{array}$ & $\begin{array}{c}\text { No } \\
x^{2}(1, \mathrm{~N}=78) \\
=66.46^{*}\end{array}$ & $\begin{array}{c}\text { No } \\
x^{2}(1, \mathrm{~N}=75) \\
=16.33^{*}\end{array}$ & 3 \\
\hline IAP & Geography & Cultural Features & $\begin{array}{c}\text { Yes } \\
x^{2}(1, \mathrm{~N}=93)= \\
48.27^{*}\end{array}$ & $\begin{array}{c}\text { No } \\
x^{2}(1, \mathrm{~N}=80) \\
=11.25^{*}\end{array}$ & $\begin{array}{c}\text { Yes } \\
x^{2}(1, \mathrm{~N}=55) \\
=4.09^{*}\end{array}$ & 2 \\
\hline IAP & Geography & Parallels and Meridians & $\begin{array}{c}\text { Yes } \\
x^{2}(1, N=103)= \\
14.77 *\end{array}$ & $\begin{array}{c}\text { No } \\
x^{2}(1, \mathrm{~N}=71) \\
=55.90 *\end{array}$ & $\begin{array}{c}\text { No } \\
x^{2}(1, \mathrm{~N}=67) \\
=14.34^{*}\end{array}$ & 3 \\
\hline IAP & Geography & Range & $\begin{array}{c}\text { Yes } \\
x^{2}(1, \mathrm{~N}=98)= \\
62.08^{*}\end{array}$ & $\begin{array}{c}\text { No } \\
x^{2}(1, \mathrm{~N}=88) \\
=10.23^{*}\end{array}$ & $\begin{array}{c}\text { No preference } \\
x^{2}(1, \mathrm{~N}=59) \\
=2.86\end{array}$ & 2 \\
\hline IAP & Geography & Water Features & $\begin{array}{c}\text { Yes } \\
x^{2}(1, \mathrm{~N}=105)= \\
68.81^{*}\end{array}$ & $\begin{array}{c}\text { No } \\
x^{2}(1, \mathrm{~N}=95) \\
=12.89^{*}\end{array}$ & $\begin{array}{c}\text { Yes } \\
x^{2}(1, \mathrm{~N}=65) \\
=4.45^{*}\end{array}$ & 2 \\
\hline IAP & Geography & Terrain Contours & $\begin{array}{c}\text { Yes } \\
x^{2}(1, \mathrm{~N}=106)= \\
102.04^{*}\end{array}$ & $\begin{array}{c}\text { Yes } \\
x^{2}(1, \mathrm{~N}= \\
105)=5.95^{*}\end{array}$ & & 1 \\
\hline IAP & Geography & $\begin{array}{l}\text { Terrain Contour } \\
\text { Elevations }\end{array}$ & $\begin{array}{c}\text { Yes } \\
x^{2}(1, \mathrm{~N}=106)= \\
102.04^{*}\end{array}$ & $\begin{array}{c}\text { No preference } \\
x^{2}(1, \mathrm{~N}= \\
105)=0.77 \\
\end{array}$ & & 1 \\
\hline IAP & Geography & Contour Interval Legend & $\begin{array}{c}\text { Yes } \\
x^{2}(1, \mathrm{~N}=104)= \\
71.12^{*}\end{array}$ & $\begin{array}{l}\text { No } \\
x^{2}(1, \mathrm{~N}=95) \\
=41.78^{*}\end{array}$ & $\begin{array}{l}\text { No preference } \\
x^{2}(1, N=79) \\
\quad=2.85\end{array}$ & $\begin{array}{l}\text { Not Yet } \\
\text { Determined }\end{array}$ \\
\hline IAP & Geography & Spot Elevations & $\begin{array}{c}\text { Yes } \\
x^{2}(1, N=101)= \\
97.04^{*}\end{array}$ & $\begin{array}{c}\text { No preference } \\
x^{2}(1, N= \\
100)=0.36 \\
\end{array}$ & & $\begin{array}{l}\text { Not Yet } \\
\text { Determined }\end{array}$ \\
\hline IAP & Geography & $\begin{array}{l}\text { Highest Reference Point } \\
\text { (within neat lines) }\end{array}$ & $\begin{array}{c}\text { Yes } \\
x^{2}(1, \mathrm{~N}=106)= \\
98.15^{*}\end{array}$ & $\begin{array}{c}\text { No preference } \\
x^{2}(1, N= \\
104)=1.38 \\
\end{array}$ & & $\begin{array}{l}\text { Not Yet } \\
\text { Determined }\end{array}$ \\
\hline IAP & Geography & $\begin{array}{l}\text { International Boundaries } \\
\text { (higher criticality where } \\
\text { appropriate) }\end{array}$ & $\begin{array}{c}\text { Yes } \\
x^{2}(1, \mathrm{~N}=104)= \\
77.88^{*}\end{array}$ & $\begin{array}{c}\text { No } \\
x^{2}(1, \mathrm{~N}=97) \\
=5.45^{*}\end{array}$ & $\begin{array}{c}\text { Yes } \\
x^{2}(1, \mathrm{~N}=60) \\
=6.67^{*}\end{array}$ & 2 \\
\hline IAP & Geography & $\begin{array}{l}\text { Visual Landmarks (when } \\
\text { not required for } \\
\text { navigation) }\end{array}$ & $\begin{array}{c}\text { Yes } \\
x^{2}(1, \mathrm{~N}=106)= \\
76.42^{*}\end{array}$ & $\begin{array}{c}\text { No } \\
x^{2}(1, \mathrm{~N}=98) \\
=55.88^{*}\end{array}$ & $\begin{array}{l}\text { No preference } \\
x^{2}(1, \mathrm{~N}=86) \\
=0.42\end{array}$ & 2 \\
\hline IAP & Geography & $\begin{array}{l}\text { Visual Landmark Label } \\
\text { (when not required for } \\
\text { navigation) }\end{array}$ & $\begin{array}{c}\text { Yes } \\
x^{2}(1, \mathrm{~N}=106)= \\
76.42^{*}\end{array}$ & $\begin{array}{c}\text { No } \\
x^{2}(1, \mathrm{~N}=98) \\
=55.88^{*}\end{array}$ & $\begin{array}{l}\text { No preference } \\
x^{2}(1, \mathrm{~N}=86) \\
\quad=0.74\end{array}$ & 3 \\
\hline IAP & Obstacles & $\begin{array}{l}\text { Obstacle Symbols and } \\
\text { Elevation }\end{array}$ & $\begin{array}{c}\text { Yes } \\
x^{2}(1, \mathrm{~N}=105)= \\
101.04^{*}\end{array}$ & $\begin{array}{c}\text { No preference } \\
x^{2}(1, N= \\
104)=2.46\end{array}$ & & 1 \\
\hline
\end{tabular}




\begin{tabular}{|c|c|c|c|c|c|c|}
\hline $\begin{array}{l}\text { Chart } \\
\text { Type }\end{array}$ & $\begin{array}{l}\text { Information } \\
\text { Category }\end{array}$ & Information Element & $\begin{array}{l}\text { Q1: Should it be } \\
\text { displayed to } \\
\text { execute } \\
\text { procedure? }\end{array}$ & $\begin{array}{l}\text { Q2: Displayed } \\
\text { at all times? }\end{array}$ & $\begin{array}{l}\text { Q3: Displayed } \\
\text { Initially? }\end{array}$ & $\begin{array}{l}\text { Importance } \\
\text { Level }\end{array}$ \\
\hline IAP & Obstacles & $\begin{array}{l}\text { Obstacle Heights and } \\
\text { related datum }\end{array}$ & $\begin{array}{c}\text { Yes } \\
x^{2}(1, \mathrm{~N}=103)= \\
95.16^{*}\end{array}$ & $\begin{array}{c}\text { No preference } \\
x^{2}(1, N= \\
101)=0.49\end{array}$ & & 1 \\
\hline IAP & $\begin{array}{l}\text { Minimum } \\
\text { Area/Sector } \\
\text { Altitudes }\end{array}$ & $\begin{array}{l}\text { MSA Reference } \\
\text { Point/Center }\end{array}$ & $\begin{array}{c}\text { Yes } \\
x^{2}(1, \mathrm{~N}=106)= \\
106.00^{*}\end{array}$ & $\begin{array}{c}\text { No } \\
x^{2}(1, \mathrm{~N}= \\
106)=6.38^{*}\end{array}$ & $\begin{array}{c}\text { Yes } \\
x^{2}(1, \mathrm{~N}=66) \\
=32.06^{*}\end{array}$ & 2 \\
\hline IAP & $\begin{array}{l}\text { Minimum } \\
\text { Area/Sector } \\
\text { Altitudes }\end{array}$ & $\begin{array}{l}\text { MSA Distance when } \\
\text { other than 25nm }\end{array}$ & $\begin{array}{c}\text { Yes } \\
x^{2}(1, \mathrm{~N}=105)= \\
101.04^{*}\end{array}$ & $\begin{array}{c}\text { No } \\
x^{2}(1, \mathrm{~N}= \\
104)=15.38^{*}\end{array}$ & $\begin{array}{c}\text { Yes } \\
x^{2}(1, \mathrm{~N}=72) \\
=29.39 *\end{array}$ & 2 \\
\hline IAP & $\begin{array}{l}\text { Minimum } \\
\text { Area/Sector } \\
\text { Altitudes }\end{array}$ & MSA Sector Radials & $\begin{array}{c}\text { Yes } \\
x^{2}(1, \mathrm{~N}=105)= \\
105.00^{*}\end{array}$ & $\begin{array}{c}\text { No } \\
x^{2}(1, \mathrm{~N}= \\
105)=8.01^{*}\end{array}$ & $\begin{array}{c}\text { Yes } \\
x^{2}(1, \mathrm{~N}=67) \\
=27.60^{*}\end{array}$ & 2 \\
\hline IAP & $\begin{array}{l}\text { Minimum } \\
\text { Area/Sector } \\
\text { Altitudes }\end{array}$ & $\begin{array}{l}\text { MSA Minimum } \\
\text { Altitudes }\end{array}$ & $\begin{array}{c}\text { Yes } \\
x^{2}(1, \mathrm{~N}=105)= \\
105.00^{*}\end{array}$ & $\begin{array}{c}\text { No preference } \\
x^{2}(1, N= \\
105)=0.09\end{array}$ & & 1 \\
\hline IAP & $\begin{array}{l}\text { Minimum } \\
\text { Area/Sector } \\
\text { Altitudes }\end{array}$ & $\begin{array}{l}\text { Minimum Radar } \\
\text { Altitudes and Sectors }\end{array}$ & $\begin{array}{c}\text { Yes } \\
x^{2}(1, \mathrm{~N}=104)= \\
88.62 *\end{array}$ & $\begin{array}{c}\text { No } \\
x^{2}(1, \mathrm{~N}= \\
100)=12.96^{*}\end{array}$ & $\begin{array}{c}\text { Yes } \\
x^{2}(1, \mathrm{~N}=68) \\
=11.53^{*}\end{array}$ & 2 \\
\hline IAP & Primary Airport & Runway Layouts & $\begin{array}{c}\text { Yes } \\
x^{2}(1, \mathrm{~N}=106)= \\
102.04^{*}\end{array}$ & $\begin{array}{c}\text { Yes } \\
x^{2}(1, \mathrm{~N}= \\
105)=10.37 *\end{array}$ & & 1 \\
\hline IAP & Primary Airport & $\begin{array}{l}\text { Landing Runway } \\
\text { Number }\end{array}$ & $\begin{array}{c}\text { Yes } \\
x^{2}(1, \mathrm{~N}=106)= \\
98.15^{*}\end{array}$ & $\begin{array}{c}\text { Yes } \\
x^{2}(1, \mathrm{~N}= \\
104)=41.88^{*}\end{array}$ & & 1 \\
\hline IAP & Primary Airport & Other Runway Numbers & $\begin{array}{c}\text { Yes } \\
x^{2}(1, \mathrm{~N}=105)= \\
85.95^{*}\end{array}$ & $\begin{array}{c}\text { No } \\
x^{2}(1, \mathrm{~N}= \\
100)=29.16^{*}\end{array}$ & $\begin{array}{c}\text { Yes } \\
x^{2}(1, \mathrm{~N}=77) \\
=24.01^{*}\end{array}$ & 2 \\
\hline IAP & Primary Airport & $\begin{array}{l}\text { Straight-in Landing } \\
\text { Runway Length }\end{array}$ & $\begin{array}{c}\text { Yes } \\
x^{2}(1, \mathrm{~N}=105)= \\
97.15^{*}\end{array}$ & $\begin{array}{c}\text { No preference } \\
x^{2}(1, \mathrm{~N}= \\
103)=0.01 \\
\end{array}$ & & $\begin{array}{l}\text { Not Yet } \\
\text { Determined }\end{array}$ \\
\hline IAP & Primary Airport & $\begin{array}{l}\text { TDZE/Threshold } \\
\text { Elevation for Landing } \\
\text { Runway }\end{array}$ & $\begin{array}{c}\text { Yes } \\
x^{2}(1, \mathrm{~N}=106)= \\
106.00^{*}\end{array}$ & $\begin{array}{c}\text { Yes } \\
x^{2}(1, \mathrm{~N}= \\
106)=15.09 *\end{array}$ & & 1 \\
\hline IAP & Primary Airport & $\begin{array}{l}\text { Glide Path Intercept } \\
\text { Point }\end{array}$ & $\begin{array}{c}\text { Yes } \\
x^{2}(1, \mathrm{~N}=106)= \\
106.00^{*}\end{array}$ & $\begin{array}{c}\text { Yes } \\
x^{2}(1, \mathrm{~N}= \\
106)=27.51^{*}\end{array}$ & & 1 \\
\hline IAP & Primary Airport & $\begin{array}{l}\text { Runway Location in } \\
\text { Profile View }\end{array}$ & $\begin{array}{c}\text { Yes } \\
x^{2}(1, \mathrm{~N}=105)= \\
105.00^{*}\end{array}$ & $\begin{array}{c}\text { Yes } \\
x^{2}(1, \mathrm{~N}= \\
105)=13.04^{*}\end{array}$ & & 1 \\
\hline IAP & $\begin{array}{l}\text { Secondary } \\
\text { Airports }\end{array}$ & $\begin{array}{l}\text { Source Doc - Runway } \\
\text { Layouts and Name }\end{array}$ & $\begin{array}{c}\text { Yes } \\
x^{2}(1, \mathrm{~N}=95)= \\
69.06^{*}\end{array}$ & $\begin{array}{c}\text { No } \\
x^{2}(1, \mathrm{~N}=88) \\
=65.64^{*}\end{array}$ & $\begin{array}{c}\text { No preference } \\
x^{2}(1, N=82) \\
=2.39\end{array}$ & $\begin{array}{l}\text { Not Yet } \\
\text { Determined }\end{array}$ \\
\hline IAP & $\begin{array}{l}\text { Secondary } \\
\text { Airports }\end{array}$ & $\begin{array}{l}\text { IFR Airports in Plan } \\
\text { View }\end{array}$ & $\begin{array}{c}\text { Yes } \\
x^{2}(1, \mathrm{~N}=102)= \\
65.92^{*}\end{array}$ & $\begin{array}{c}\text { No } \\
x^{2}(1, \mathrm{~N}=92) \\
=47.35^{*}\end{array}$ & $\begin{array}{c}\text { Yes } \\
x^{2}(1, \mathrm{~N}=79) \\
=7.91^{*}\end{array}$ & 2 \\
\hline
\end{tabular}




\begin{tabular}{|c|c|c|c|c|c|c|}
\hline $\begin{array}{l}\text { Chart } \\
\text { Type }\end{array}$ & $\begin{array}{l}\text { Information } \\
\text { Category }\end{array}$ & Information Element & $\begin{array}{l}\text { Q1: Should it be } \\
\text { displayed to } \\
\text { execute } \\
\text { procedure? }\end{array}$ & $\begin{array}{l}\text { Q2: Displayed } \\
\text { at all times? }\end{array}$ & $\begin{array}{l}\text { Q3: Displayed } \\
\text { Initially? }\end{array}$ & $\begin{array}{l}\text { Importance } \\
\text { Level }\end{array}$ \\
\hline IAP & $\begin{array}{l}\text { Secondary } \\
\text { Airports }\end{array}$ & $\begin{array}{l}\text { VFR Airports within } \\
\text { Specified Distance of the } \\
\text { Approach Track }\end{array}$ & $\begin{array}{c}\text { Yes } \\
x^{2}(1, \mathrm{~N}=102)= \\
50.82^{*}\end{array}$ & $\begin{array}{c}\text { No } \\
x^{2}(1, \mathrm{~N}=87) \\
=54.72^{*}\end{array}$ & $\begin{array}{l}\text { No preference } \\
x^{2}(1, \mathrm{~N}=78) \\
=0.00\end{array}$ & $\begin{array}{l}\text { Not Yet } \\
\text { Determined }\end{array}$ \\
\hline IAP & Navigation & FIR/UIR Boundaries & $\begin{array}{c}\text { Yes } \\
x^{2}(1, \mathrm{~N}=93)= \\
40.01^{*}\end{array}$ & $\begin{array}{c}\text { No } \\
x^{2}(1, \mathrm{~N}=77) \\
=26.30^{*}\end{array}$ & $\begin{array}{l}\text { No preference } \\
x^{2}(1, \mathrm{~N}=61) \\
=1.33\end{array}$ & 2 \\
\hline IAP & Navigation & $\begin{array}{l}\text { Terminal Arrival Area } \\
\text { (TAA) }\end{array}$ & $\begin{array}{c}\text { Yes } \\
x^{2}(1, \mathrm{~N}=97)= \\
57.99 *\end{array}$ & $\begin{array}{c}\text { No } \\
x^{2}(1, \mathrm{~N}=86) \\
=36.47^{*}\end{array}$ & $\begin{array}{c}\text { Yes } \\
x^{2}(1, \mathrm{~N}=71) \\
=7.45^{*}\end{array}$ & 2 \\
\hline IAP & Navigation & $\begin{array}{l}\text { Prohibited, Restricted } \\
\text { and Danger Airspace } \\
\text { Graphic }\end{array}$ & $\begin{array}{c}\text { Yes } \\
x^{2}(1, \mathrm{~N}=106)= \\
98.15^{*}\end{array}$ & $\begin{array}{c}\text { Yes } \\
x^{2}(1, \mathrm{~N}= \\
104)=8.65^{*}\end{array}$ & & 1 \\
\hline IAP & Navigation & $\begin{array}{l}\text { Prohibited, Restricted } \\
\text { and Danger Airspace } \\
\text { Label }\end{array}$ & $\begin{array}{c}\text { Yes } \\
x^{2}(1, \mathrm{~N}=106)= \\
86.94^{*}\end{array}$ & $\begin{array}{c}\text { No preference } \\
x^{2}(1, N= \\
101)=1.67\end{array}$ & & 1 \\
\hline IAP & Navigation & $\begin{array}{l}\text { Prohibited, Restricted } \\
\text { and Danger Airspace } \\
\text { Narrative }\end{array}$ & $\begin{array}{c}\text { Yes } \\
x^{2}(1, \mathrm{~N}=106)= \\
83.36^{*}\end{array}$ & $\begin{array}{c}\text { No } \\
x^{2}(1, \mathrm{~N}= \\
100)=27.04^{*}\end{array}$ & $\begin{array}{l}\text { No preference } \\
x^{2}(1, \mathrm{~N}=76) \\
\quad=2.58\end{array}$ & 2 \\
\hline IAP & Navigation & $\begin{array}{l}\text { Special Use Airspace - } \\
\text { Other }\end{array}$ & $\begin{array}{c}\text { Yes } \\
x^{2}(1, \mathrm{~N}=106)= \\
76.42^{*}\end{array}$ & $\begin{array}{c}\text { No } \\
x^{2}(1, \mathrm{~N}=98) \\
=39.22^{*}\end{array}$ & $\begin{array}{c}\text { Yes } \\
x^{2}(1, \mathrm{~N}=80) \\
=22.05^{*}\end{array}$ & 2 \\
\hline IAP & Navigation & General Notes & $\begin{array}{c}\text { Yes } \\
x^{2}(1, \mathrm{~N}=102)= \\
75.92 *\end{array}$ & $\begin{array}{c}\text { No } \\
x^{2}(1, \mathrm{~N}=95) \\
=91.04 *\end{array}$ & $\begin{array}{c}\text { Yes } \\
x^{2}(1, \mathrm{~N}=94) \\
=7.19 *\end{array}$ & 2 \\
\hline IAP & Navigation & Procedural Data Notes & $\begin{array}{c}\text { Yes } \\
x^{2}(1, \mathrm{~N}=104)= \\
88.62 *\end{array}$ & $\begin{array}{c}\text { No } \\
x^{2}(1, \mathrm{~N}= \\
100)=67.24^{*}\end{array}$ & $\begin{array}{c}\text { Yes } \\
x^{2}(1, \mathrm{~N}=91) \\
=18.47^{*}\end{array}$ & 2 \\
\hline IAP & Navigation & Transition Level & $\begin{array}{c}\text { Yes } \\
x^{2}(1, \mathrm{~N}=102)= \\
86.63^{*}\end{array}$ & $\begin{array}{c}\text { No } \\
x^{2}(1, \mathrm{~N}=98) \\
=16.33^{*}\end{array}$ & $\begin{array}{c}\text { Yes } \\
x^{2}(1, \mathrm{~N}=69) \\
=12.19^{*}\end{array}$ & 2 \\
\hline IAP & Navigation & Transition Altitude & $\begin{array}{c}\text { Yes } \\
x^{2}(1, N=102)= \\
86.63^{*}\end{array}$ & $\begin{array}{c}\text { No } \\
x^{2}(1, \mathrm{~N}=98) \\
=18.00^{*}\end{array}$ & $\begin{array}{c}\text { Yes } \\
x^{2}(1, \mathrm{~N}=70) \\
=14.63^{*}\end{array}$ & 2 \\
\hline IAP & Navigation & Fix Symbol & $\begin{array}{c}\text { Yes } \\
x^{2}(1, \mathrm{~N}=106)= \\
106.00^{*}\end{array}$ & $\begin{array}{c}\text { Yes } \\
x^{2}(1, \mathrm{~N}= \\
106)=54.49^{*}\end{array}$ & & 1 \\
\hline IAP & Navigation & Fix Name/Identifier & $\begin{array}{c}\text { Yes } \\
x^{2}(1, \mathrm{~N}=106)= \\
106.00^{*}\end{array}$ & $\begin{array}{c}\text { Yes } \\
x^{2}(1, \mathrm{~N}= \\
106)=63.43^{*}\end{array}$ & & 1 \\
\hline
\end{tabular}




\begin{tabular}{|c|c|c|c|c|c|c|}
\hline $\begin{array}{l}\text { Chart } \\
\text { Type }\end{array}$ & $\begin{array}{l}\text { Information } \\
\text { Category }\end{array}$ & Information Element & $\begin{array}{l}\text { Q1: Should it be } \\
\text { displayed to } \\
\text { execute } \\
\text { procedure? }\end{array}$ & $\begin{array}{l}\text { Q2: Displayed } \\
\text { at all times? }\end{array}$ & $\begin{array}{l}\text { Q3: Displayed } \\
\text { Initially? }\end{array}$ & $\begin{array}{l}\text { Importance } \\
\text { Level }\end{array}$ \\
\hline IAP & Navigation & Fix Altitude & $\begin{array}{c}\text { Yes } \\
x^{2}(1, \mathrm{~N}=106)= \\
106.00^{*}\end{array}$ & $\begin{array}{c}\text { Yes } \\
x^{2}(1, \mathrm{~N}= \\
106)=69.77^{*}\end{array}$ & & 1 \\
\hline IAP & Navigation & Step-Down Fix Altitude & $\begin{array}{c}\text { Yes } \\
x^{2}(1, \mathrm{~N}=106)= \\
106.00^{*}\end{array}$ & $\begin{array}{c}\text { Yes } \\
x^{2}(1, \mathrm{~N}= \\
106)=69.77 *\end{array}$ & & 1 \\
\hline IAP & Navigation & $\begin{array}{l}\text { Step-Down Fix } \\
\text { Formation }\end{array}$ & $\begin{array}{c}\text { Yes } \\
x^{2}(1, \mathrm{~N}=97)= \\
97.00^{*}\end{array}$ & $\begin{array}{c}\text { Yes } \\
x^{2}(1, \mathrm{~N}=97) \\
=17.33^{*}\end{array}$ & & 1 \\
\hline IAP & Navigation & Fix Formation & $\begin{array}{c}\text { Yes } \\
x^{2}(1, \mathrm{~N}=95)= \\
95.00^{*}\end{array}$ & $\begin{array}{c}\text { Yes } \\
x^{2}(1, \mathrm{~N}=95) \\
=4.64^{*}\end{array}$ & & 1 \\
\hline IAP & Navigation & $\begin{array}{l}\text { All appropriate navaid } \\
\text { symbols }\end{array}$ & $\begin{array}{c}\text { Yes } \\
x^{2}(1, \mathrm{~N}=106)= \\
106.00^{*}\end{array}$ & $\begin{array}{c}\text { Yes } \\
x^{2}(1, \mathrm{~N}= \\
106)=13.62 *\end{array}$ & & 1 \\
\hline IAP & Navigation & Lead Radial & $\begin{array}{c}\text { Yes } \\
x^{2}(1, \mathrm{~N}=105)= \\
97.15^{*}\end{array}$ & $\begin{array}{c}\text { No preference } \\
x^{2}(1, \mathrm{~N}= \\
103)=0.48\end{array}$ & & 1 \\
\hline IAP & Navigation & $\begin{array}{l}\text { Localizer Magnetic } \\
\text { Course }\end{array}$ & $\begin{array}{c}\text { Yes } \\
x^{2}(1, \mathrm{~N}=106)= \\
106.00^{*}\end{array}$ & $\begin{array}{c}\text { Yes } \\
x^{2}(1, \mathrm{~N}= \\
106)=43.62 *\end{array}$ & & 1 \\
\hline IAP & Navigation & $\begin{array}{l}\text { FAF (e.g., Maltese } \\
\text { Cross) }\end{array}$ & $\begin{array}{c}\text { Yes } \\
x^{2}(1, \mathrm{~N}=106)= \\
106.00^{*}\end{array}$ & $\begin{array}{c}\text { Yes } \\
x^{2}(1, \mathrm{~N}= \\
106)=83.36^{*}\end{array}$ & & 1 \\
\hline IAP & Navigation & $\begin{array}{l}\text { GS Intercept Altitude } \\
\text { (MSL) }\end{array}$ & $\begin{array}{c}\text { Yes } \\
x^{2}(1, \mathrm{~N}=105)= \\
105.00^{*}\end{array}$ & $\begin{array}{c}\text { Yes } \\
x^{2}(1, \mathrm{~N}= \\
105)=50.75^{*}\end{array}$ & & 1 \\
\hline IAP & Navigation & Glide Slope Angle & $\begin{array}{c}\text { Yes } \\
x^{2}(1, \mathrm{~N}=105)= \\
101.04^{*}\end{array}$ & $\begin{array}{c}\text { No } \\
x^{2}(1, \mathrm{~N}= \\
104)=12.46^{*}\end{array}$ & $\begin{array}{c}\text { Yes } \\
x^{2}(1, \mathrm{~N}=70) \\
=35.71^{*}\end{array}$ & 2 \\
\hline IAP & Navigation & $\begin{array}{l}\text { VNAV Intercept } \\
\text { Altitude (MSL) }\end{array}$ & $\begin{array}{c}\text { Yes } \\
x^{2}(1, \mathrm{~N}=104)= \\
104.00^{*}\end{array}$ & $\begin{array}{c}\text { Yes } \\
x^{2}(1, \mathrm{~N}= \\
104)=13.88^{*}\end{array}$ & & 1 \\
\hline IAP & Navigation & $\begin{array}{l}\text { FAF Crossing Altitude } \\
\text { (MSL) (HAT) }\end{array}$ & $\begin{array}{c}\text { Yes } \\
x^{2}(1, N=106)= \\
98.15^{*}\end{array}$ & $\begin{array}{c}\text { Yes } \\
x^{2}(1, \mathrm{~N}= \\
104)=58.50^{*}\end{array}$ & & 1 \\
\hline IAP & Navigation & $\begin{array}{l}\text { GS Intercept Altitude } \\
\text { (Above Airport) (QFE) }\end{array}$ & $\begin{array}{c}\text { Yes } \\
x^{2}(1, \mathrm{~N}=104)= \\
81.38^{*}\end{array}$ & $\begin{array}{c}\text { Yes } \\
x^{2}(1, \mathrm{~N}=98) \\
=6.90^{*}\end{array}$ & & 1 \\
\hline IAP & Navigation & $\begin{array}{l}\text { VNAV Intercept } \\
\text { Altitude (Above Airport) } \\
\text { (QFE) }\end{array}$ & $\begin{array}{c}\text { Yes } \\
x^{2}(1, \mathrm{~N}=103)= \\
80.40^{*}\end{array}$ & $\begin{array}{l}\text { No preference } \\
x^{2}(1, N=97) \\
=0.51\end{array}$ & & 1 \\
\hline IAP & Navigation & VNAV Angle & $\begin{array}{c}\text { Yes } \\
x^{2}(1, \mathrm{~N}=105)= \\
97.15^{*}\end{array}$ & $\begin{array}{c}\text { No } \\
x^{2}(1, \mathrm{~N}= \\
103)=23.31^{*}\end{array}$ & $\begin{array}{c}\text { Yes } \\
x^{2}(1, \mathrm{~N}=76) \\
=32.89^{*}\end{array}$ & 2 \\
\hline
\end{tabular}




\begin{tabular}{|c|c|c|c|c|c|c|}
\hline $\begin{array}{l}\text { Chart } \\
\text { Type }\end{array}$ & $\begin{array}{l}\text { Information } \\
\text { Category }\end{array}$ & Information Element & $\begin{array}{l}\text { Q1: Should it be } \\
\text { displayed to } \\
\text { execute } \\
\text { procedure? }\end{array}$ & $\begin{array}{l}\text { Q2: Displayed } \\
\text { at all times? }\end{array}$ & $\begin{array}{l}\text { Q3: Displayed } \\
\text { Initially? }\end{array}$ & $\begin{array}{l}\text { Importance } \\
\text { Level }\end{array}$ \\
\hline IAP & Navigation & $\begin{array}{l}\text { Rate of Descent (feet per } \\
\text { minute) }\end{array}$ & $\begin{array}{c}\text { Yes } \\
x^{2}(1, \mathrm{~N}=106)= \\
98.15^{*}\end{array}$ & $\begin{array}{c}\text { No } \\
x^{2}(1, \mathrm{~N}= \\
104)=52.65^{*}\end{array}$ & $\begin{array}{c}\text { Yes } \\
x^{2}(1, \mathrm{~N}=89) \\
=20.78^{*}\end{array}$ & 2 \\
\hline IAP & Navigation & $\begin{array}{l}\text { Threshold Crossing } \\
\text { Height }\end{array}$ & $\begin{array}{c}\text { Yes } \\
x^{2}(1, \mathrm{~N}=106)= \\
90.60^{*}\end{array}$ & $\begin{array}{c}\text { No } \\
x^{2}(1, \mathrm{~N}= \\
102)=14.16^{*}\end{array}$ & $\begin{array}{c}\text { Yes } \\
x^{2}(1, \mathrm{~N}=70) \\
=16.51^{*}\end{array}$ & 2 \\
\hline IAP & Navigation Aids & Localizer Frequency & $\begin{array}{c}\text { Yes } \\
x^{2}(1, \mathrm{~N}=106)= \\
106.00^{*}\end{array}$ & $\begin{array}{c}\text { No preference } \\
x^{2}(1, N= \\
106)=0.60\end{array}$ & & 1 \\
\hline IAP & Navigation Aids & Localizer Identifier & $\begin{array}{c}\text { Yes } \\
x^{2}(1, \mathrm{~N}=106)= \\
98.15^{*}\end{array}$ & $\begin{array}{c}\text { No } \\
x^{2}(1, \mathrm{~N}= \\
104)=6.50^{*}\end{array}$ & $\begin{array}{c}\text { Yes } \\
x^{2}(1, \mathrm{~N}=65) \\
=33.98^{*}\end{array}$ & 2 \\
\hline IAP & Navigation Aids & Localizer Morse Code & $\begin{array}{c}\text { Yes } \\
x^{2}(1, \mathrm{~N}=105)= \\
82.37^{*}\end{array}$ & $\begin{array}{c}\text { No } \\
x^{2}(1, \mathrm{~N}=99) \\
=45.34^{*}\end{array}$ & $\begin{array}{l}\text { No preference } \\
x^{2}(1, N=83) \\
=2.71\end{array}$ & $\begin{array}{l}\text { Not Yet } \\
\text { Determined }\end{array}$ \\
\hline IAP & Navigation Aids & $\begin{array}{l}\text { Primary Approach } \\
\text { Localizer Symbol }\end{array}$ & $\begin{array}{c}\text { Yes } \\
x^{2}(1, \mathrm{~N}=101)= \\
85.63^{*}\end{array}$ & $\begin{array}{l}\text { No preference } \\
x^{2}(1, N=97) \\
=0.84\end{array}$ & & 2 \\
\hline IAP & Navigation Aids & $\begin{array}{l}\text { Simultaneous Parallel } \\
\text { Localizer Symbol }\end{array}$ & $\begin{array}{c}\text { Yes } \\
x^{2}(1, \mathrm{~N}=101)= \\
85.63^{*}\end{array}$ & $\begin{array}{c}\text { No } \\
x^{2}(1, \mathrm{~N}=97) \\
=35.89^{*}\end{array}$ & $\begin{array}{c}\text { Yes } \\
x^{2}(1, \mathrm{~N}=78) \\
=18.51^{*}\end{array}$ & 2 \\
\hline IAP & Navigation Aids & $\begin{array}{l}\text { Localizer for Intersection } \\
\text { Formations }\end{array}$ & $\begin{array}{c}\text { Yes } \\
x^{2}(1, \mathrm{~N}=93)= \\
81.39 *\end{array}$ & $\begin{array}{c}\text { No } \\
x^{2}(1, \mathrm{~N}=90) \\
=17.78^{*}\end{array}$ & $\begin{array}{c}\text { Yes } \\
x^{2}(1, \mathrm{~N}=65) \\
=31.15^{*}\end{array}$ & 2 \\
\hline IAP & Navigation Aids & $\begin{array}{l}\text { Localizer Front Course } \\
\text { for Back Course } \\
\text { Approaches }\end{array}$ & $\begin{array}{c}\text { Yes } \\
x^{2}(1, \mathrm{~N}=104)= \\
81.38^{*}\end{array}$ & $\begin{array}{l}\text { No preference } \\
x^{2}(1, N=98) \\
\quad=3.31\end{array}$ & $\begin{array}{c}\text { Yes } \\
x^{2}(1, N=58) \\
=24.90^{*}\end{array}$ & $\begin{array}{l}\text { Not Yet } \\
\text { Determined }\end{array}$ \\
\hline IAP & Navigation Aids & $\begin{array}{l}\text { WAAS/SBAS - } \\
\text { LAAS/GBAS Channel }\end{array}$ & $\begin{array}{c}\text { Yes } \\
x^{2}(1, \mathrm{~N}=93)= \\
54.20^{*}\end{array}$ & $\begin{array}{c}\text { No } \\
x^{2}(1, \mathrm{~N}=82) \\
=32.98^{*}\end{array}$ & $\begin{array}{c}\text { Yes } \\
x^{2}(1, \mathrm{~N}=67) \\
=6.58^{*}\end{array}$ & 2 \\
\hline IAP & Navigation Aids & Marker Beacon Symbols & $\begin{array}{c}\text { Yes } \\
x^{2}(1, \mathrm{~N}=106)= \\
76.42^{*}\end{array}$ & $\begin{array}{c}\text { No } \\
x^{2}(1, \mathrm{~N}=98) \\
=19.76^{*}\end{array}$ & $\begin{array}{c}\text { Yes } \\
x^{2}(1, \mathrm{~N}=71) \\
=17.25^{*}\end{array}$ & 2 \\
\hline IAP & Navigation Aids & $\begin{array}{l}\text { Marker Beacon Labels } \\
\text { (i.e., OM,MM,IM) }\end{array}$ & $\begin{array}{c}\text { Yes } \\
x^{2}(1, \mathrm{~N}=106)= \\
79.85^{*}\end{array}$ & $\begin{array}{c}\text { No } \\
x^{2}(1, \mathrm{~N}=99) \\
=30.56^{*}\end{array}$ & $\begin{array}{c}\text { Yes } \\
x^{2}(1, \mathrm{~N}=77) \\
=6.87^{*}\end{array}$ & 2 \\
\hline IAP & $\begin{array}{l}\text { Procedure } \\
\text { Navaid }\end{array}$ & Navaid Symbol & $\begin{array}{c}\text { Yes } \\
x^{2}(1, \mathrm{~N}=106)= \\
106.00^{*}\end{array}$ & $\begin{array}{c}\text { Yes } \\
x^{2}(1, \mathrm{~N}= \\
106)=10.91 *\end{array}$ & & 1 \\
\hline IAP & $\begin{array}{l}\text { Procedure } \\
\text { Navaid }\end{array}$ & Navaid Name & $\begin{array}{c}\text { Yes } \\
x^{2}(1, \mathrm{~N}=106)= \\
106.00^{*}\end{array}$ & $\begin{array}{c}\text { No preference } \\
x^{2}(1, N= \\
106)=1.36\end{array}$ & & 1 \\
\hline
\end{tabular}




\begin{tabular}{|c|c|c|c|c|c|c|}
\hline $\begin{array}{l}\text { Chart } \\
\text { Type }\end{array}$ & $\begin{array}{l}\text { Information } \\
\text { Category }\end{array}$ & Information Element & $\begin{array}{l}\text { Q1: Should it be } \\
\text { displayed to } \\
\text { execute } \\
\text { procedure? }\end{array}$ & $\begin{array}{l}\text { Q2: Displayed } \\
\text { at all times? }\end{array}$ & $\begin{array}{l}\text { Q3: Displayed } \\
\text { Initially? }\end{array}$ & $\begin{array}{l}\text { Importance } \\
\text { Level }\end{array}$ \\
\hline IAP & $\begin{array}{l}\text { Procedure } \\
\text { Navaid }\end{array}$ & Navaid Identifier & $\begin{array}{c}\text { Yes } \\
x^{2}(1, \mathrm{~N}=106)= \\
106.00^{*}\end{array}$ & $\begin{array}{c}\text { No preference } \\
x^{2}(1, N= \\
106)=0.60\end{array}$ & & 2 \\
\hline IAP & $\begin{array}{l}\text { Procedure } \\
\text { Navaid }\end{array}$ & Navaid Frequency & $\begin{array}{c}\text { Yes } \\
x^{2}(1, \mathrm{~N}=106)= \\
106.00^{*}\end{array}$ & $\begin{array}{c}\text { No preference } \\
x^{2}(1, \mathrm{~N}= \\
106)=0.94\end{array}$ & & 1 \\
\hline IAP & $\begin{array}{l}\text { Procedure } \\
\text { Navaid }\end{array}$ & Navaid Morse Code & $\begin{array}{c}\text { Yes } \\
x^{2}(1, N=106)= \\
69.77^{*}\end{array}$ & $\begin{array}{c}\text { No } \\
x^{2}(1, \mathrm{~N}=96) \\
=57.04^{*}\end{array}$ & $\begin{array}{l}\text { No preference } \\
\begin{array}{c}x^{2}(1, \mathrm{~N}=85) \\
=0.01\end{array}\end{array}$ & $\begin{array}{l}\text { Not Yet } \\
\text { Determined }\end{array}$ \\
\hline IAP & $\begin{array}{l}\text { Procedure } \\
\text { Navaid }\end{array}$ & DME Availability & $\begin{array}{c}\text { Yes } \\
x^{2}(1, \mathrm{~N}=106)= \\
86.94^{*}\end{array}$ & $\begin{array}{c}\text { No } \\
x^{2}(1, \mathrm{~N}= \\
101)=13.55^{*}\end{array}$ & $\begin{array}{c}\text { Yes } \\
x^{2}(1, \mathrm{~N}=69) \\
=29.35^{*}\end{array}$ & 2 \\
\hline IAP & $\begin{array}{l}\text { Procedure } \\
\text { Navaid }\end{array}$ & Navaid Class & $\begin{array}{c}\text { Yes } \\
x^{2}(1, \mathrm{~N}=105)= \\
42.75^{*}\end{array}$ & $\begin{array}{c}\text { No } \\
x^{2}(1, \mathrm{~N}=86) \\
=60.28^{*}\end{array}$ & $\begin{array}{l}\text { No preference } \\
\begin{array}{c}x^{2}(1, N=79) \\
=3.66\end{array}\end{array}$ & 3 \\
\hline IAP & $\begin{array}{l}\text { Procedure } \\
\text { Navaid }\end{array}$ & $\begin{array}{l}\text { Navaid } \\
\text { Latitude/Longitude }\end{array}$ & $\begin{array}{c}\text { Yes } \\
x^{2}(1, N=106)= \\
10.91^{*}\end{array}$ & $\begin{array}{c}\text { No } \\
x^{2}(1, \mathrm{~N}=70) \\
=58.51^{*}\end{array}$ & $\begin{array}{c}\text { No } \\
x^{2}(1, \mathrm{~N}=67) \\
=14.34^{*}\end{array}$ & 3 \\
\hline IAP & $\begin{array}{l}\text { Landing } \\
\text { Minimums }\end{array}$ & $\begin{array}{l}\text { Minimum Descent } \\
\text { Altitude (MDA) }\end{array}$ & $\begin{array}{c}\text { Yes } \\
x^{2}(1, \mathrm{~N}=106)= \\
106.00^{*}\end{array}$ & $\begin{array}{c}\text { Yes } \\
x^{2}(1, \mathrm{~N}= \\
106)=66.57^{*}\end{array}$ & & 1 \\
\hline IAP & $\begin{array}{l}\text { Landing } \\
\text { Minimums }\end{array}$ & $\begin{array}{l}\text { Minimum Descent } \\
\text { Height (MDH) }\end{array}$ & $\begin{array}{c}\text { Yes } \\
x^{2}(1, \mathrm{~N}=106)= \\
106.00^{*}\end{array}$ & $\begin{array}{c}\text { Yes } \\
x^{2}(1, \mathrm{~N}= \\
106)=41.09^{*}\end{array}$ & & 1 \\
\hline IAP & $\begin{array}{l}\text { Landing } \\
\text { Minimums }\end{array}$ & $\begin{array}{l}\text { Height Above Airport } \\
\text { (HAA) }\end{array}$ & $\begin{array}{c}\text { Yes } \\
x^{2}(1, \mathrm{~N}=105)= \\
101.04^{*}\end{array}$ & $\begin{array}{c}\text { No preference } \\
x^{2}(1, N= \\
104)=0.04 \\
\end{array}$ & & 1 \\
\hline IAP & $\begin{array}{l}\text { Landing } \\
\text { Minimums }\end{array}$ & $\begin{array}{l}\text { CAT I Decision Altitude } \\
\text { (DA) }\end{array}$ & $\begin{array}{c}\text { Yes } \\
x^{2}(1, \mathrm{~N}=104)= \\
104.00^{*}\end{array}$ & $\begin{array}{c}\text { Yes } \\
x^{2}(1, \mathrm{~N}= \\
104)=52.65^{*}\end{array}$ & & 1 \\
\hline IAP & $\begin{array}{l}\text { Landing } \\
\text { Minimums }\end{array}$ & $\begin{array}{l}\text { CAT II Decision } \\
\text { Altitude (DA) }\end{array}$ & $\begin{array}{c}\text { Yes } \\
x^{2}(1, \mathrm{~N}=103)= \\
103.00^{*}\end{array}$ & $\begin{array}{c}\text { Yes } \\
x^{2}(1, \mathrm{~N}= \\
103)=25.25^{*}\end{array}$ & & 1 \\
\hline IAP & $\begin{array}{l}\text { Landing } \\
\text { Minimums }\end{array}$ & Decision Height (DH) & $\begin{array}{c}\text { Yes } \\
x^{2}(1, \mathrm{~N}=105)= \\
105.00^{*}\end{array}$ & $\begin{array}{c}\text { Yes } \\
x^{2}(1, \mathrm{~N}= \\
105)=62.49^{*}\end{array}$ & & 1 \\
\hline IAP & $\begin{array}{l}\text { Landing } \\
\text { Minimums }\end{array}$ & $\begin{array}{l}\text { CAT II Radio Altimeter } \\
\text { (RA) }\end{array}$ & $\begin{array}{c}\text { Yes } \\
x^{2}(1, \mathrm{~N}=98)= \\
98.00^{*}\end{array}$ & $\begin{array}{c}\text { Yes } \\
x^{2}(1, \mathrm{~N}=98) \\
=5.88^{*}\end{array}$ & & 1 \\
\hline IAP & $\begin{array}{l}\text { Landing } \\
\text { Minimums }\end{array}$ & Visibility Requirement & $\begin{array}{c}\text { Yes } \\
x^{2}(1, \mathrm{~N}=106)= \\
102.04^{*}\end{array}$ & $\begin{array}{c}\text { No preference } \\
x^{2}(1, N= \\
105)=0.01 \\
\end{array}$ & & 1 \\
\hline IAP & $\begin{array}{l}\text { Missed } \\
\text { Approach }\end{array}$ & $\begin{array}{l}\text { Missed Approach } \\
\text { Instructions }\end{array}$ & $\begin{array}{c}\text { Yes } \\
x^{2}(1, \mathrm{~N}=106)= \\
106.00^{*}\end{array}$ & $\begin{array}{c}\text { Yes } \\
x^{2}(1, \mathrm{~N}= \\
106)=13.62 *\end{array}$ & & 1 \\
\hline
\end{tabular}




\begin{tabular}{|c|c|c|c|c|c|c|}
\hline $\begin{array}{l}\text { Chart } \\
\text { Type }\end{array}$ & $\begin{array}{l}\text { Information } \\
\text { Category }\end{array}$ & Information Element & $\begin{array}{l}\text { Q1: Should it be } \\
\text { displayed to } \\
\text { execute } \\
\text { procedure? }\end{array}$ & $\begin{array}{l}\text { Q2: Displayed } \\
\text { at all times? }\end{array}$ & $\begin{array}{l}\text { Q3: Displayed } \\
\text { Initially? }\end{array}$ & $\begin{array}{l}\text { Importance } \\
\text { Level }\end{array}$ \\
\hline IAP & $\begin{array}{l}\text { Missed } \\
\text { Approach }\end{array}$ & $\begin{array}{l}\text { Name of Missed } \\
\text { Approach Holding Fix }\end{array}$ & $\begin{array}{c}\text { Yes } \\
x^{2}(1, \mathrm{~N}=106)= \\
106.00^{*}\end{array}$ & $\begin{array}{c}\text { Yes } \\
x^{2}(1, \mathrm{~N}= \\
106)=4.57 *\end{array}$ & & 1 \\
\hline IAP & $\begin{array}{l}\text { Missed } \\
\text { Approach }\end{array}$ & $\begin{array}{l}\text { Missed Approach } \\
\text { Holding Pattern }\end{array}$ & $\begin{array}{c}\text { Yes } \\
x^{2}(1, \mathrm{~N}=105)= \\
105.00^{*}\end{array}$ & $\begin{array}{c}\text { No preference } \\
x^{2}(1, N= \\
105)=3.44 \\
\end{array}$ & & 1 \\
\hline IAP & $\begin{array}{l}\text { Missed } \\
\text { Approach }\end{array}$ & Location of MAP & $\begin{array}{c}\text { Yes } \\
x^{2}(1, N=106)= \\
102.04^{*}\end{array}$ & $\begin{array}{c}\text { Yes } \\
x^{2}(1, \mathrm{~N}= \\
105)=24.77^{*}\end{array}$ & & 1 \\
\hline IAP & $\begin{array}{l}\text { Missed } \\
\text { Approach }\end{array}$ & Time From FAF to MAP & $\begin{array}{c}\text { Yes } \\
x^{2}(1, \mathrm{~N}=106)= \\
94.34^{*}\end{array}$ & $\begin{array}{c}\text { No } \\
x^{2}(1, \mathrm{~N}= \\
103)=14.77 *\end{array}$ & $\begin{array}{c}\text { Yes } \\
x^{2}(1, \mathrm{~N}=71) \\
=8.80^{*}\end{array}$ & $\begin{array}{l}\text { Not Yet } \\
\text { Determined }\end{array}$ \\
\hline IAP & $\begin{array}{l}\text { Missed } \\
\text { Approach }\end{array}$ & $\begin{array}{l}\text { Distance From FAF to } \\
\text { MAP }\end{array}$ & $\begin{array}{c}\text { Yes } \\
x^{2}(1, \mathrm{~N}=106)= \\
98.15^{*}\end{array}$ & $\begin{array}{c}\text { No } \\
x^{2}(1, \mathrm{~N}= \\
104)=3.85^{*}\end{array}$ & $\begin{array}{c}\text { Yes } \\
x^{2}(1, \mathrm{~N}=62) \\
=14.52^{*}\end{array}$ & 2 \\
\hline IAP & $\begin{array}{l}\text { Missed } \\
\text { Approach }\end{array}$ & $\begin{array}{l}\text { Fix Name/Identifier at } \\
\text { MAP }\end{array}$ & $\begin{array}{c}\text { Yes } \\
x^{2}(1, \mathrm{~N}=106)= \\
98.15^{*}\end{array}$ & $\begin{array}{c}\text { No preference } \\
x^{2}(1, N= \\
104)=3.12\end{array}$ & & 1 \\
\hline IAP & Holding Pattern & $\begin{array}{l}\text { Holding Pattern } \\
\text { Depiction }\end{array}$ & $\begin{array}{c}\text { Yes } \\
x^{2}(1, \mathrm{~N}=106)= \\
106.00^{*}\end{array}$ & $\begin{array}{c}\text { No preference } \\
x^{2}(1, N= \\
106)=2.42 \\
\end{array}$ & & 1 \\
\hline IAP & Holding Pattern & Holding Pattern Courses & $\begin{array}{c}\text { Yes } \\
x^{2}(1, \mathrm{~N}=106)= \\
102.04^{*}\end{array}$ & $\begin{array}{c}\text { No preference } \\
x^{2}(1, N= \\
105)=0.24\end{array}$ & & 1 \\
\hline IAP & Holding Pattern & $\begin{array}{l}\text { Holding Pattern Leg } \\
\text { Length }\end{array}$ & $\begin{array}{c}\text { Yes } \\
x^{2}(1, \mathrm{~N}=106)= \\
102.04^{*}\end{array}$ & $\begin{array}{c}\text { No } \\
x^{2}(1, \mathrm{~N}= \\
105)=6.94 *\end{array}$ & $\begin{array}{c}\text { Yes } \\
x^{2}(1, \mathrm{~N}=66) \\
=13.64^{*}\end{array}$ & 2 \\
\hline IAP & Holding Pattern & Holding Pattern Time & $\begin{array}{c}\text { Yes } \\
x^{2}(1, \mathrm{~N}=106)= \\
98.15^{*}\end{array}$ & $\begin{array}{c}\text { No } \\
x^{2}(1, \mathrm{~N}= \\
104)=12.46^{*}\end{array}$ & $\begin{array}{c}\text { Yes } \\
x^{2}(1, \mathrm{~N}=70) \\
=11.20^{*}\end{array}$ & 2 \\
\hline IAP & Holding Pattern & Holding Pattern Speed & $\begin{array}{c}\text { Yes } \\
x^{2}(1, \mathrm{~N}=106)= \\
94.34^{*}\end{array}$ & $\begin{array}{c}\text { No } \\
x^{2}(1, \mathrm{~N}= \\
103)=27.27^{*}\end{array}$ & $\begin{array}{c}\text { Yes } \\
x^{2}(1, \mathrm{~N}=78) \\
=13.13^{*}\end{array}$ & 2 \\
\hline IAP & Holding Pattern & Holding Pattern Altitude & $\begin{array}{c}\text { Yes } \\
x^{2}(1, \mathrm{~N}=106)= \\
102.04^{*}\end{array}$ & $\begin{array}{c}\text { No preference } \\
x^{2}(1, N= \\
105)=0.47 \\
\end{array}$ & & 1 \\
\hline IAP & Communications & ATIS Arrival Frequency & $\begin{array}{c}\text { Yes } \\
x^{2}(1, \mathrm{~N}=106)= \\
102.04^{*}\end{array}$ & $\begin{array}{c}\text { No } \\
x^{2}(1, \mathrm{~N}= \\
105)=45.34^{*}\end{array}$ & $\begin{array}{c}\text { Yes } \\
x^{2}(1, \mathrm{~N}=87) \\
=15.74^{*}\end{array}$ & 2 \\
\hline IAP & Communications & $\begin{array}{l}\text { Departure Control } \\
\text { Frequency }\end{array}$ & $\begin{array}{c}\text { Yes } \\
x^{2}(1, \mathrm{~N}=106)= \\
86.94^{*}\end{array}$ & $\begin{array}{c}\text { No } \\
x^{2}(1, \mathrm{~N}= \\
101)=16.64^{*}\end{array}$ & $\begin{array}{c}\text { Yes } \\
x^{2}(1, \mathrm{~N}=71) \\
=13.54^{*}\end{array}$ & 2 \\
\hline IAP & Communications & Tower Frequency & $\begin{array}{c}\text { Yes } \\
x^{2}(1, \mathrm{~N}=106)= \\
106.00^{*}\end{array}$ & $\begin{array}{c}\text { Yes } \\
x^{2}(1, \mathrm{~N}= \\
106)=6.38^{*}\end{array}$ & & 1 \\
\hline
\end{tabular}




\begin{tabular}{|c|c|c|c|c|c|c|}
\hline $\begin{array}{l}\text { Chart } \\
\text { Type }\end{array}$ & $\begin{array}{l}\text { Information } \\
\text { Category }\end{array}$ & Information Element & $\begin{array}{l}\text { Q1: Should it be } \\
\text { displayed to } \\
\text { execute } \\
\text { procedure? }\end{array}$ & $\begin{array}{l}\text { Q2: Displayed } \\
\text { at all times? }\end{array}$ & $\begin{array}{l}\text { Q3: Displayed } \\
\text { Initially? }\end{array}$ & $\begin{array}{l}\text { Importance } \\
\text { Level }\end{array}$ \\
\hline IAP & Communications & Ground Frequency & $\begin{array}{c}\text { Yes } \\
x^{2}(1, \mathrm{~N}=106)= \\
98.15^{*}\end{array}$ & $\begin{array}{c}\text { No preference } \\
x^{2}(1, N= \\
104)=0.62\end{array}$ & & 2 \\
\hline IAP & Communications & Approach Frequency & $\begin{array}{c}\text { Yes } \\
x^{2}(1, \mathrm{~N}=106)= \\
94.34^{*}\end{array}$ & $\begin{array}{c}\text { No preference } \\
x^{2}(1, N= \\
103)=0.01\end{array}$ & & $\begin{array}{l}\text { Not Yet } \\
\text { Determined }\end{array}$ \\
\hline IAP & Communications & Clearance Frequency & $\begin{array}{c}\text { Yes } \\
x^{2}(1, N=106)= \\
66.57^{*}\end{array}$ & $\begin{array}{c}\text { No } \\
x^{2}(1, \mathrm{~N}=95) \\
=44.47^{*}\end{array}$ & $\begin{array}{c}\text { Yes } \\
x^{2}(1, \mathrm{~N}=80) \\
=4.05^{*}\end{array}$ & 2 \\
\hline IAP & Communications & $\begin{array}{l}\text { ATIS Departure } \\
\text { Frequency }\end{array}$ & $\begin{array}{c}\text { Yes } \\
x^{2}(1, \mathrm{~N}=106)= \\
66.57^{*}\end{array}$ & $\begin{array}{c}\text { No } \\
x^{2}(1, \mathrm{~N}=95) \\
=47.25^{*}\end{array}$ & $\begin{array}{l}\text { No preference } \\
x^{2}(1, \mathrm{~N}=81) \\
=2.78\end{array}$ & 2 \\
\hline IAP & Communications & Helicopter Frequency & $\begin{array}{c}\text { Yes } \\
x^{2}(1, \mathrm{~N}=92)= \\
25.04^{*}\end{array}$ & $\begin{array}{c}\text { No } \\
x^{2}(1, \mathrm{~N}=70) \\
=51.43^{*}\end{array}$ & $\begin{array}{c}\text { No } \\
x^{2}(1, \mathrm{~N}=65) \\
=5.55^{*}\end{array}$ & 3 \\
\hline IAP & Navigation & Procedure Track & $\begin{array}{c}\text { Yes } \\
x^{2}(1, N=105)= \\
105.00^{*}\end{array}$ & $\begin{array}{c}\text { Yes } \\
x^{2}(1, \mathrm{~N}= \\
105)=40.24^{*}\end{array}$ & & 1 \\
\hline IAP & Navigation & $\begin{array}{l}\text { Procedure Magnetic } \\
\text { Course }\end{array}$ & $\begin{array}{c}\text { Yes } \\
x^{2}(1, \mathrm{~N}=104)= \\
100.04^{*}\end{array}$ & $\begin{array}{c}\text { Yes } \\
x^{2}(1, \mathrm{~N}= \\
103)=11.89 *\end{array}$ & & 1 \\
\hline IAP & Navigation & Procedure Track Altitude & $\begin{array}{c}\text { Yes } \\
x^{2}(1, \mathrm{~N}=105)= \\
105.00^{*}\end{array}$ & $\begin{array}{c}\text { Yes } \\
x^{2}(1, \mathrm{~N}= \\
105)=26.75^{*}\end{array}$ & & 1 \\
\hline IAP & Navigation & Procedure Track Mileage & $\begin{array}{c}\text { Yes } \\
x^{2}(1, N=105)= \\
101.04^{*}\end{array}$ & $\begin{array}{c}\text { No preference } \\
x^{2}(1, N= \\
104)=0.96 \\
\end{array}$ & & 1 \\
\hline IAP & Navigation & $\begin{array}{l}\text { Procedure Turn } \\
\text { Outbound Course }\end{array}$ & $\begin{array}{c}\text { Yes } \\
x^{2}(1, \mathrm{~N}=106)= \\
106.00^{*}\end{array}$ & $\begin{array}{c}\text { Yes } \\
x^{2}(1, \mathrm{~N}= \\
106)=8.49 *\end{array}$ & & 1 \\
\hline IAP & Navigation & Procedure Turn Altitude & $\begin{array}{c}\text { Yes } \\
x^{2}(1, \mathrm{~N}=105)= \\
105.00^{*}\end{array}$ & $\begin{array}{c}\text { Yes } \\
x^{2}(1, \mathrm{~N}= \\
105)=21.04^{*}\end{array}$ & & 1 \\
\hline IAP & Navigation & $\begin{array}{l}\text { Procedure Turn Distance } \\
\text { Limit }\end{array}$ & $\begin{array}{c}\text { Yes } \\
x^{2}(1, \mathrm{~N}=105)= \\
105.00^{*}\end{array}$ & $\begin{array}{c}\text { No preference } \\
x^{2}(1, N= \\
105)=2.14 \\
\end{array}$ & & 1 \\
\hline IAP & $\begin{array}{l}\text { Navaids in the } \\
\text { Vicinity of the } \\
\text { Procedure }\end{array}$ & Navaid Symbol & $\begin{array}{c}\text { Yes } \\
x^{2}(1, \mathrm{~N}=106)= \\
94.34^{*}\end{array}$ & $\begin{array}{c}\text { No preference } \\
x^{2}(1, N= \\
103)=0.79\end{array}$ & & $\begin{array}{l}\text { Not Yet } \\
\text { Determined }\end{array}$ \\
\hline IAP & $\begin{array}{l}\text { Navaids in the } \\
\text { Vicinity of the } \\
\text { Procedure }\end{array}$ & Navaid Name & $\begin{array}{c}\text { Yes } \\
x^{2}(1, \mathrm{~N}=106)= \\
90.60^{*}\end{array}$ & $\begin{array}{c}\text { No } \\
x^{2}(1, \mathrm{~N}= \\
102)=11.33^{*}\end{array}$ & $\begin{array}{c}\text { Yes } \\
x^{2}(1, \mathrm{~N}=68) \\
=19.06^{*}\end{array}$ & 2 \\
\hline IAP & $\begin{array}{l}\text { Navaids in the } \\
\text { Vicinity of the } \\
\text { Procedure }\end{array}$ & Navaid Identifier & $\begin{array}{c}\text { Yes } \\
x^{2}(1, \mathrm{~N}=105)= \\
97.15^{*}\end{array}$ & $\begin{array}{c}\text { No } \\
x^{2}(1, \mathrm{~N}= \\
103)=13.29 *\end{array}$ & $\begin{array}{c}\text { Yes } \\
x^{2}(1, \mathrm{~N}=70) \\
=9.66^{*}\end{array}$ & 2 \\
\hline
\end{tabular}




\begin{tabular}{|c|c|c|c|c|c|c|}
\hline $\begin{array}{l}\text { Chart } \\
\text { Type }\end{array}$ & $\begin{array}{l}\text { Information } \\
\text { Category }\end{array}$ & Information Element & $\begin{array}{l}\text { Q1: Should it be } \\
\text { displayed to } \\
\text { execute } \\
\text { procedure? }\end{array}$ & $\begin{array}{l}\text { Q2: Displayed } \\
\text { at all times? }\end{array}$ & $\begin{array}{l}\text { Q3: Displayed } \\
\text { Initially? }\end{array}$ & $\begin{array}{l}\text { Importance } \\
\text { Level }\end{array}$ \\
\hline IAP & $\begin{array}{l}\text { Navaids in the } \\
\text { Vicinity of the } \\
\text { Procedure }\end{array}$ & Navaid Frequency & $\begin{array}{c}\text { Yes } \\
x^{2}(1, \mathrm{~N}=106)= \\
94.34^{*}\end{array}$ & $\begin{array}{c}\text { No } \\
x^{2}(1, \mathrm{~N}= \\
103)=14.77 *\end{array}$ & $\begin{array}{c}\text { Yes } \\
x^{2}(1, \mathrm{~N}=71) \\
=8.80^{*}\end{array}$ & $\begin{array}{l}\text { Not Yet } \\
\text { Determined }\end{array}$ \\
\hline IAP & $\begin{array}{l}\text { Navaids in the } \\
\text { Vicinity of the } \\
\text { Procedure }\end{array}$ & Navaid Morse Code & $\begin{array}{c}\text { Yes } \\
x^{2}(1, \mathrm{~N}=106)= \\
51.66^{*}\end{array}$ & $\begin{array}{c}\text { No } \\
x^{2}(1, \mathrm{~N}=90) \\
=71.11^{*}\end{array}$ & $\begin{array}{l}\text { No preference } \\
x^{2}(1, N=85) \\
=0.58\end{array}$ & $\begin{array}{l}\text { Not Yet } \\
\text { Determined }\end{array}$ \\
\hline IAP & $\begin{array}{l}\text { Navaids in the } \\
\text { Vicinity of the } \\
\text { Procedure }\end{array}$ & DME Availability & $\begin{array}{c}\text { Yes } \\
x^{2}(1, \mathrm{~N}=105)= \\
78.87^{*}\end{array}$ & $\begin{array}{c}\text { No } \\
x^{2}(1, N=98) \\
=62.08^{*}\end{array}$ & $\begin{array}{c}\text { Yes } \\
x^{2}(1, \mathrm{~N}=88) \\
=10.23^{*}\end{array}$ & 2 \\
\hline IAP & $\begin{array}{l}\text { Navaids in the } \\
\text { Vicinity of the } \\
\text { Procedure }\end{array}$ & Navaid Class & $\begin{array}{c}\text { Yes } \\
x^{2}(1, \mathrm{~N}=106)= \\
29.58^{*}\end{array}$ & $\begin{array}{c}\text { No } \\
x^{2}(1, \mathrm{~N}=81) \\
=65.79^{*}\end{array}$ & $\begin{array}{l}\text { No preference } \\
x^{2}(1, \mathrm{~N}=77) \\
=2.19\end{array}$ & 3 \\
\hline IAP & $\begin{array}{l}\text { Navaids in the } \\
\text { Vicinity of the } \\
\text { Procedure }\end{array}$ & $\begin{array}{l}\text { Navaid } \\
\text { Latitude/Longitude }\end{array}$ & $\begin{array}{c}\text { Yes } \\
x^{2}(1, \mathrm{~N}=106)= \\
12.23^{*}\end{array}$ & $\begin{array}{c}\text { No } \\
x^{2}(1, \mathrm{~N}=71) \\
=67.06^{*}\end{array}$ & $\begin{array}{c}\text { No } \\
x^{2}(1, \mathrm{~N}=70) \\
=8.23^{*}\end{array}$ & 3 \\
\hline IFR & Identification & $\begin{array}{l}\text { Revision Date (i.e., Start } \\
\text { and Finish) }\end{array}$ & $\begin{array}{c}\text { Yes } \\
x^{2}(1, \mathrm{~N}=106)= \\
83.36^{*}\end{array}$ & $\begin{array}{c}\text { No } \\
x^{2}(1, \mathrm{~N}= \\
100)=64.00^{*}\end{array}$ & $\begin{array}{c}\text { Yes } \\
x^{2}(1, \mathrm{~N}=90) \\
=14.40^{*}\end{array}$ & 2 \\
\hline IFR & Identification & $\begin{array}{l}\text { Chart Description (e.g., } \\
\text { High, Low, etc.) }\end{array}$ & $\begin{array}{c}\text { Yes } \\
x^{2}(1, \mathrm{~N}=106)= \\
83.36^{*}\end{array}$ & $\begin{array}{c}\text { No } \\
x^{2}(1, \mathrm{~N}= \\
100)=16.00^{*}\end{array}$ & $\begin{array}{c}\text { Yes } \\
x^{2}(1, \mathrm{~N}=70) \\
=32.91^{*}\end{array}$ & 2 \\
\hline IFR & Geography & Range & $\begin{array}{c}\text { Yes } \\
x^{2}(1, \mathrm{~N}=103)= \\
83.97^{*}\end{array}$ & $\begin{array}{c}\text { No } \\
x^{2}(1, \mathrm{~N}=98) \\
=16.33^{*}\end{array}$ & $\begin{array}{c}\text { Yes } \\
x^{2}(1, \mathrm{~N}=69) \\
=15.78^{*}\end{array}$ & 2 \\
\hline IFR & Geography & $\begin{array}{l}\text { Indication of Area Chart } \\
\text { Coverage }\end{array}$ & $\begin{array}{c}\text { Yes } \\
x^{2}(1, \mathrm{~N}=104)= \\
84.96^{*}\end{array}$ & $\begin{array}{c}\text { No } \\
x^{2}(1, \mathrm{~N}=99) \\
=37.59 *\end{array}$ & $\begin{array}{c}\text { Yes } \\
x^{2}(1, \mathrm{~N}=80) \\
=22.05^{*}\end{array}$ & 2 \\
\hline IFR & Geography & Parallels and Meridians & $\begin{array}{c}\text { Yes } \\
x^{2}(1, \mathrm{~N}=102)= \\
75.92 *\end{array}$ & $\begin{array}{c}\text { No } \\
x^{2}(1, \mathrm{~N}=95) \\
=39.17^{*}\end{array}$ & $\begin{array}{c}\text { Yes } \\
x^{2}(1, \mathrm{~N}=78) \\
=5.13^{*}\end{array}$ & 2 \\
\hline IFR & Geography & Water Features & $\begin{array}{c}\text { Yes } \\
x^{2}(1, \mathrm{~N}=106)= \\
79.85^{*}\end{array}$ & $\begin{array}{c}\text { No } \\
x^{2}(1, \mathrm{~N}=99) \\
=11.00^{*}\end{array}$ & $\begin{array}{c}\text { Yes } \\
x^{2}(1, \mathrm{~N}=66) \\
=17.52^{*}\end{array}$ & 2 \\
\hline IFR & Geography & Contour Interval Legend & $\begin{array}{c}\text { Yes } \\
x^{2}(1, N=106)= \\
76.42 *\end{array}$ & $\begin{array}{c}\text { No } \\
x^{2}(1, \mathrm{~N}=98) \\
=47.18^{*}\end{array}$ & $\begin{array}{c}\text { No preference } \\
x^{2}(1, \mathrm{~N}=83) \\
=2.04\end{array}$ & 2 \\
\hline IFR & Geography & Spot Elevations & $\begin{array}{c}\text { Yes } \\
x^{2}(1, \mathrm{~N}=104)= \\
88.62 * \\
\end{array}$ & $\begin{array}{c}\text { No } \\
x^{2}(1, \mathrm{~N}= \\
100)=5.76^{*}\end{array}$ & $\begin{array}{c}\text { Yes } \\
x^{2}(1, \mathrm{~N}=62) \\
=14.52^{*}\end{array}$ & 2 \\
\hline IFR & Obstacle & $\begin{array}{l}\text { Obstacle Symbols and } \\
\text { Elevation (e.g., man } \\
\text { made, exceptionally } \\
\text { high) }\end{array}$ & $\begin{array}{c}\text { Yes } \\
x^{2}(1, \mathrm{~N}=106)= \\
102.04^{*}\end{array}$ & $\begin{array}{c}\text { No preference } \\
x^{2}(1, \mathrm{~N}= \\
105)=0.01\end{array}$ & & 1 \\
\hline
\end{tabular}




\begin{tabular}{|c|c|c|c|c|c|c|}
\hline $\begin{array}{l}\text { Chart } \\
\text { Type }\end{array}$ & $\begin{array}{l}\text { Information } \\
\text { Category }\end{array}$ & Information Element & $\begin{array}{l}\text { Q1: Should it be } \\
\text { displayed to } \\
\text { execute } \\
\text { procedure? }\end{array}$ & $\begin{array}{l}\text { Q2: Displayed } \\
\text { at all times? }\end{array}$ & $\begin{array}{l}\text { Q3: Displayed } \\
\text { Initially? }\end{array}$ & $\begin{array}{l}\text { Importance } \\
\text { Level }\end{array}$ \\
\hline IFR & $\begin{array}{l}\text { Minimum } \\
\text { Area/Sector } \\
\text { Altitudes }\end{array}$ & $\begin{array}{l}\text { Area Minimum Altitudes } \\
\text { - OROCA, Sector } \\
\text { Altitudes (Grid MORA } \\
\text { Outside of US) }\end{array}$ & $\begin{array}{c}\text { Yes } \\
x^{2}(1, \mathrm{~N}=106)= \\
106.00^{*}\end{array}$ & $\begin{array}{c}\text { No preference } \\
x^{2}(1, N= \\
106)=0.34\end{array}$ & & 1 \\
\hline IFR & Communications & $\begin{array}{l}\text { Graphical Portrayal of } \\
\text { Radio Frequency Sector } \\
\text { Boundaries }\end{array}$ & $\begin{array}{c}\text { Yes } \\
x^{2}(1, \mathrm{~N}=106)= \\
106.00^{*}\end{array}$ & $\begin{array}{c}\text { No } \\
x^{2}(1, \mathrm{~N}= \\
106)=16.64^{*}\end{array}$ & $\begin{array}{c}\text { Yes } \\
x^{2}(1, \mathrm{~N}=74) \\
=5.41^{*}\end{array}$ & 2 \\
\hline IFR & Communications & $\begin{array}{l}\text { Voice Frequencies } \\
\text { associated with Navaid } \\
\text { Facility Boxes }\end{array}$ & $\begin{array}{c}\text { Yes } \\
x^{2}(1, \mathrm{~N}=105)= \\
101.04^{*}\end{array}$ & $\begin{array}{c}\text { No } \\
x^{2}(1, \mathrm{~N}= \\
104)=26.00^{*}\end{array}$ & $\begin{array}{l}\text { No preference } \\
x^{2}(1, \mathrm{~N}=78) \\
\quad=0.21\end{array}$ & 2 \\
\hline IFR & Communications & $\begin{array}{l}\text { FIR/UIR, Control, } \\
\text { ARTCC, etc., Frequency } \\
\text { Boxes }\end{array}$ & $\begin{array}{c}\text { Yes } \\
x^{2}(1, \mathrm{~N}=104)= \\
100.04^{*}\end{array}$ & $\begin{array}{c}\text { No } \\
x^{2}(1, \mathrm{~N}= \\
103)=7.08^{*}\end{array}$ & $\begin{array}{l}\text { No preference } \\
\begin{array}{l}x^{2}(1, \mathrm{~N}=65) \\
\quad=3.46\end{array}\end{array}$ & 2 \\
\hline IFR & Communications & $\begin{array}{l}\text { Call and Frequencies of } \\
\text { In-Flight Weather } \\
\text { Stations }\end{array}$ & $\begin{array}{c}\text { Yes } \\
x^{2}(1, \mathrm{~N}=105)= \\
89.61^{*}\end{array}$ & $\begin{array}{c}\text { No } \\
x^{2}(1, \mathrm{~N}= \\
101)=44.45^{*}\end{array}$ & $\begin{array}{c}\text { No } \\
x^{2}(1, \mathrm{~N}=84) \\
=10.71^{*}\end{array}$ & 3 \\
\hline IFR & Communications & $\begin{array}{l}\text { Company Specific } \\
\text { Frequencies (tailored } \\
\text { communications) }\end{array}$ & $\begin{array}{c}\text { Yes } \\
x^{2}(1, \mathrm{~N}=102)= \\
56.63^{*}\end{array}$ & $\begin{array}{c}\text { No } \\
x^{2}(1, \mathrm{~N}=89) \\
=66.62 *\end{array}$ & $\begin{array}{l}\text { No preference } \\
x^{2}(1, \mathrm{~N}=83) \\
=2.71\end{array}$ & 3 \\
\hline IFR & $\begin{array}{l}\text { Airspace } \\
\text { Boundaries }\end{array}$ & $\begin{array}{l}\text { Unit Providing Area } \\
\text { Control Service }\end{array}$ & $\begin{array}{c}\text { Yes } \\
x^{2}(1, \mathrm{~N}=102)= \\
98.04^{*}\end{array}$ & $\begin{array}{c}\text { No } \\
x^{2}(1, \mathrm{~N}= \\
101)=15.06^{*} \\
\end{array}$ & $\begin{array}{c}\text { Yes } \\
x^{2}(1, \mathrm{~N}=70) \\
=4.63^{*}\end{array}$ & 2 \\
\hline IFR & $\begin{array}{l}\text { Airspace } \\
\text { Boundaries }\end{array}$ & $\begin{array}{l}\text { Control Zone (CTR) } \\
\text { Boundaries }\end{array}$ & $\begin{array}{c}\text { Yes } \\
x^{2}(1, \mathrm{~N}=103)= \\
95.16^{*}\end{array}$ & $\begin{array}{c}\text { No } \\
x^{2}(1, \mathrm{~N}= \\
101)=8.33^{*}\end{array}$ & $\begin{array}{c}\text { Yes } \\
x^{2}(1, \mathrm{~N}=65) \\
=6.78^{*}\end{array}$ & 2 \\
\hline IFR & $\begin{array}{l}\text { Airspace } \\
\text { Boundaries }\end{array}$ & Name of CTR & $\begin{array}{c}\text { Yes } \\
x^{2}(1, \mathrm{~N}=104)= \\
84.96^{*}\end{array}$ & $\begin{array}{c}\text { No } \\
x^{2}(1, \mathrm{~N}=99) \\
=13.83^{*}\end{array}$ & $\begin{array}{c}\text { Yes } \\
x^{2}(1, \mathrm{~N}=68) \\
=9.94^{*}\end{array}$ & 2 \\
\hline IFR & $\begin{array}{l}\text { Airspace } \\
\text { Boundaries }\end{array}$ & CTR Vertical Limits & $\begin{array}{c}\text { Yes } \\
x^{2}(1, \mathrm{~N}=101)= \\
81.99^{*}\end{array}$ & $\begin{array}{c}\text { No } \\
x^{2}(1, \mathrm{~N}=96) \\
=32.67^{*}\end{array}$ & $\begin{array}{l}\text { No preference } \\
x^{2}(1, \mathrm{~N}=76) \\
=1.32\end{array}$ & $\begin{array}{l}\text { Not Yet } \\
\text { Determined }\end{array}$ \\
\hline IFR & $\begin{array}{l}\text { Airspace } \\
\text { Boundaries }\end{array}$ & $\begin{array}{l}\text { Airspace Class Vertical } \\
\text { Limits }\end{array}$ & $\begin{array}{c}\text { Yes } \\
x^{2}(1, \mathrm{~N}=104)= \\
84.96^{*}\end{array}$ & $\begin{array}{c}\text { No } \\
x^{2}(1, \mathrm{~N}=99) \\
=30.56^{*}\end{array}$ & $\begin{array}{l}\text { No preference } \\
x^{2}(1, \mathrm{~N}=77) \\
\quad=2.19\end{array}$ & 2 \\
\hline IFR & $\begin{array}{l}\text { Airspace } \\
\text { Boundaries }\end{array}$ & Airspace Class Notes & $\begin{array}{c}\text { Yes } \\
x^{2}(1, \mathrm{~N}=104)= \\
88.62 *\end{array}$ & $\begin{array}{c}\text { No } \\
x^{2}(1, \mathrm{~N}= \\
100)=88.36^{*}\end{array}$ & $\begin{array}{l}\text { No preference } \\
x^{2}(1, \mathrm{~N}=97) \\
=2.32\end{array}$ & 2 \\
\hline
\end{tabular}




\begin{tabular}{|c|c|c|c|c|c|c|}
\hline $\begin{array}{l}\text { Chart } \\
\text { Type }\end{array}$ & $\begin{array}{l}\text { Information } \\
\text { Category }\end{array}$ & Information Element & $\begin{array}{l}\text { Q1: Should it be } \\
\text { displayed to } \\
\text { execute } \\
\text { procedure? }\end{array}$ & $\begin{array}{l}\text { Q2: Displayed } \\
\text { at all times? }\end{array}$ & $\begin{array}{l}\text { Q3: Displayed } \\
\text { Initially? }\end{array}$ & $\begin{array}{l}\text { Importance } \\
\text { Level }\end{array}$ \\
\hline IFR & $\begin{array}{l}\text { Airspace } \\
\text { Boundaries }\end{array}$ & FIR/UIR Boundaries & $\begin{array}{c}\text { Yes } \\
x^{2}(1, \mathrm{~N}=101)= \\
93.16^{*}\end{array}$ & $\begin{array}{c}\text { No } \\
x^{2}(1, \mathrm{~N}=99) \\
=5.34^{*}\end{array}$ & $\begin{array}{c}\text { Yes } \\
x^{2}(1, \mathrm{~N}=61) \\
=13.79^{*}\end{array}$ & $\begin{array}{l}\text { Not Yet } \\
\text { Determined }\end{array}$ \\
\hline IFR & $\begin{array}{l}\text { Airspace } \\
\text { Boundaries }\end{array}$ & Name of FIR/UIR & $\begin{array}{c}\text { Yes } \\
x^{2}(1, \mathrm{~N}=101)= \\
93.16^{*}\end{array}$ & $\begin{array}{c}\text { No } \\
x^{2}(1, \mathrm{~N}=99) \\
=18.68^{*}\end{array}$ & $\begin{array}{c}\text { Yes } \\
x^{2}(1, \mathrm{~N}=71) \\
=13.54^{*}\end{array}$ & 2 \\
\hline IFR & $\begin{array}{l}\text { Airspace } \\
\text { Boundaries }\end{array}$ & ID of FIR/UIR & $\begin{array}{c}\text { Yes } \\
x^{2}(1, \mathrm{~N}=101)= \\
93.16^{*}\end{array}$ & $\begin{array}{c}\text { No } \\
x^{2}(1, \mathrm{~N}=99) \\
=28.37^{*}\end{array}$ & $\begin{array}{c}\text { Yes } \\
x^{2}(1, \mathrm{~N}=76) \\
=6.37^{*}\end{array}$ & 2 \\
\hline IFR & $\begin{array}{l}\text { Airspace } \\
\text { Boundaries }\end{array}$ & Unit Providing Service & $\begin{array}{c}\text { Yes } \\
x^{2}(1, \mathrm{~N}=99)= \\
91.16^{*}\end{array}$ & $\begin{array}{c}\text { No } \\
x^{2}(1, \mathrm{~N}=97) \\
=46.28^{*}\end{array}$ & $\begin{array}{c}\text { Yes } \\
x^{2}(1, \mathrm{~N}=82) \\
=9.56^{*}\end{array}$ & 2 \\
\hline IFR & $\begin{array}{l}\text { Airspace } \\
\text { Boundaries }\end{array}$ & Name of TMA & $\begin{array}{c}\text { Yes } \\
x^{2}(1, \mathrm{~N}=84)= \\
76.19 *\end{array}$ & $\begin{array}{c}\text { No } \\
x^{2}(1, \mathrm{~N}=82) \\
=43.90^{*}\end{array}$ & $\begin{array}{c}\text { Yes } \\
x^{2}(1, \mathrm{~N}=71) \\
=4.07^{*}\end{array}$ & 2 \\
\hline IFR & $\begin{array}{l}\text { Airspace } \\
\text { Boundaries }\end{array}$ & TMA Vertical Limits & $\begin{array}{c}\text { Yes } \\
x^{2}(1, \mathrm{~N}=84)= \\
76.19 *\end{array}$ & $\begin{array}{c}\text { No } \\
x^{2}(1, \mathrm{~N}=82) \\
=49.95^{*}\end{array}$ & $\begin{array}{c}\text { Yes } \\
x^{2}(1, \mathrm{~N}=73) \\
=3.96^{*}\end{array}$ & 2 \\
\hline IFR & $\begin{array}{l}\text { Airspace } \\
\text { Boundaries }\end{array}$ & $\begin{array}{l}\text { Special Use Airspace - } \\
\text { Prohibited, Restricted, } \\
\text { Danger Boundaries }\end{array}$ & $\begin{array}{c}\text { Yes } \\
x^{2}(1, \mathrm{~N}=106)= \\
106.00^{*}\end{array}$ & $\begin{array}{c}\text { Yes } \\
x^{2}(1, \mathrm{~N}= \\
106)=9.66^{*}\end{array}$ & & 1 \\
\hline IFR & $\begin{array}{l}\text { Airspace } \\
\text { Boundaries }\end{array}$ & $\begin{array}{l}\text { Special Use Airspace ID } \\
\text { and Vertical limits }\end{array}$ & $\begin{array}{c}\text { Yes } \\
x^{2}(1, \mathrm{~N}=106)= \\
106.00^{*}\end{array}$ & $\begin{array}{c}\text { No preference } \\
x^{2}(1, \mathrm{~N}= \\
106)=2.42 \\
\end{array}$ & & 1 \\
\hline IFR & $\begin{array}{l}\text { Airspace } \\
\text { Boundaries }\end{array}$ & $\begin{array}{l}\text { Special Use Airspace - } \\
\text { Other - Boundaries }\end{array}$ & $\begin{array}{c}\text { Yes } \\
x^{2}(1, \mathrm{~N}=105)= \\
101.04^{*}\end{array}$ & $\begin{array}{c}\text { No } \\
x^{2}(1, \mathrm{~N}= \\
104)=7.54^{*}\end{array}$ & $\begin{array}{c}\text { Yes } \\
x^{2}(1, \mathrm{~N}=66) \\
=26.73^{*}\end{array}$ & $\begin{array}{l}\text { Not Yet } \\
\text { Determined }\end{array}$ \\
\hline IFR & $\begin{array}{l}\text { Airspace } \\
\text { Boundaries }\end{array}$ & $\begin{array}{l}\text { Air Defense } \\
\text { Identification Zones } \\
\text { (ADIZ) }\end{array}$ & $\begin{array}{c}\text { Yes } \\
x^{2}(1, \mathrm{~N}=106)= \\
98.15^{*}\end{array}$ & $\begin{array}{c}\text { No preference } \\
x^{2}(1, \mathrm{~N}= \\
104)=0.62 \\
\end{array}$ & & 1 \\
\hline IFR & $\begin{array}{l}\text { Airspace } \\
\text { Boundaries }\end{array}$ & $\begin{array}{l}\text { Altimeter Setting } \\
\text { Regions (i.e., lowest } \\
\text { ALT for QNH) }\end{array}$ & $\begin{array}{c}\text { Yes } \\
x^{2}(1, \mathrm{~N}=103)= \\
87.62^{*}\end{array}$ & $\begin{array}{c}\text { No } \\
x^{2}(1, \mathrm{~N}=99) \\
=18.68^{*}\end{array}$ & $\begin{array}{c}\text { Yes } \\
x^{2}(1, \mathrm{~N}=71) \\
=8.80^{*}\end{array}$ & 2 \\
\hline IFR & $\begin{array}{l}\text { Airspace } \\
\text { Boundaries }\end{array}$ & $\begin{array}{l}\text { Indication of Areas of } \\
\text { RNP, RVSM, MNPS, } \\
\text { etc., Requirements }\end{array}$ & $\begin{array}{c}\text { Yes } \\
x^{2}(1, \mathrm{~N}=100)= \\
92.16^{*}\end{array}$ & $\begin{array}{c}\text { No } \\
x^{2}(1, \mathrm{~N}=98) \\
=29.76^{*}\end{array}$ & $\begin{array}{c}\text { Yes } \\
x^{2}(1, \mathrm{~N}=76) \\
=10.32^{*}\end{array}$ & 2 \\
\hline IFR & $\begin{array}{l}\text { Airspace } \\
\text { Boundaries }\end{array}$ & Time Zone Boundaries & $\begin{array}{c}\text { Yes } \\
x^{2}(1, \mathrm{~N}=106)= \\
41.09^{*}\end{array}$ & $\begin{array}{c}\text { No } \\
x^{2}(1, \mathrm{~N}=86) \\
=53.77^{*}\end{array}$ & $\begin{array}{l}\text { No preference } \\
\begin{array}{c}x^{2}(1, N=77) \\
=2.19\end{array}\end{array}$ & 3 \\
\hline
\end{tabular}




\begin{tabular}{|c|c|c|c|c|c|c|}
\hline $\begin{array}{l}\text { Chart } \\
\text { Type }\end{array}$ & $\begin{array}{l}\text { Information } \\
\text { Category }\end{array}$ & Information Element & $\begin{array}{l}\text { Q1: Should it be } \\
\text { displayed to } \\
\text { execute } \\
\text { procedure? }\end{array}$ & $\begin{array}{l}\text { Q2: Displayed } \\
\text { at all times? }\end{array}$ & $\begin{array}{l}\text { Q3: Displayed } \\
\text { Initially? }\end{array}$ & $\begin{array}{l}\text { Importance } \\
\text { Level }\end{array}$ \\
\hline IFR & $\begin{array}{l}\text { Airspace } \\
\text { Boundaries }\end{array}$ & Airway Designator & $\begin{array}{c}\text { Yes } \\
x^{2}(1, \mathrm{~N}=104)= \\
104.00^{*}\end{array}$ & $\begin{array}{c}\text { No preference } \\
x^{2}(1, N= \\
104)=1.38 \\
\end{array}$ & & 1 \\
\hline IFR & $\begin{array}{l}\text { Airspace } \\
\text { Boundaries }\end{array}$ & $\begin{array}{l}\text { Indication of one-way } \\
\text { airways }\end{array}$ & $\begin{array}{c}\text { Yes } \\
x^{2}(1, \mathrm{~N}=106)= \\
102.04^{*}\end{array}$ & $\begin{array}{c}\text { No preference } \\
x^{2}(1, \mathrm{~N}= \\
105)=1.15 \\
\end{array}$ & & 1 \\
\hline IFR & $\begin{array}{l}\text { Airspace } \\
\text { Boundaries }\end{array}$ & Segment Mileages & $\begin{array}{c}\text { Yes } \\
x^{2}(1, \mathrm{~N}=106)= \\
102.04^{*}\end{array}$ & $\begin{array}{c}\text { No preference } \\
x^{2}(1, \mathrm{~N}= \\
105)=0.77 \\
\end{array}$ & & $\begin{array}{l}\text { Not Yet } \\
\text { Determined }\end{array}$ \\
\hline IFR & $\begin{array}{l}\text { Airspace } \\
\text { Boundaries }\end{array}$ & $\begin{array}{l}\text { Segment Upper Limit or } \\
\text { MAA }\end{array}$ & $\begin{array}{c}\text { Yes } \\
x^{2}(1, \mathrm{~N}=101)= \\
93.16^{*}\end{array}$ & $\begin{array}{c}\text { No } \\
x^{2}(1, \mathrm{~N}=99) \\
=24.25^{*}\end{array}$ & $\begin{array}{c}\text { Yes } \\
x^{2}(1, \mathrm{~N}=74) \\
=26.16^{*}\end{array}$ & 2 \\
\hline IFR & $\begin{array}{l}\text { Airspace } \\
\text { Boundaries }\end{array}$ & $\begin{array}{l}\text { Indication of MEA } \\
\text { Change at Segment End }\end{array}$ & $\begin{array}{c}\text { Yes } \\
x^{2}(1, \mathrm{~N}=104)= \\
96.15^{*}\end{array}$ & $\begin{array}{c}\text { No preference } \\
x^{2}(1, \mathrm{~N}= \\
102)=0.98\end{array}$ & & 2 \\
\hline IFR & $\begin{array}{l}\text { Airspace } \\
\text { Boundaries }\end{array}$ & Segment MORA & $\begin{array}{c}\text { Yes } \\
x^{2}(1, \mathrm{~N}=99)= \\
91.16^{*}\end{array}$ & $\begin{array}{c}\text { No } \\
x^{2}(1, \mathrm{~N}=97) \\
=5.45^{*}\end{array}$ & $\begin{array}{c}\text { Yes } \\
x^{2}(1, \mathrm{~N}=60) \\
=19.27^{*}\end{array}$ & 2 \\
\hline IFR & $\begin{array}{l}\text { Airspace } \\
\text { Boundaries }\end{array}$ & $\begin{array}{l}\text { Holding Pattern } \\
\text { restrictions }\end{array}$ & $\begin{array}{c}\text { Yes } \\
x^{2}(1, \mathrm{~N}=106)= \\
98.15^{*}\end{array}$ & $\begin{array}{c}\text { No } \\
x^{2}(1, \mathrm{~N}= \\
104)=15.38^{*}\end{array}$ & $\begin{array}{c}\text { Yes } \\
x^{2}(1, \mathrm{~N}=72) \\
=24.50^{*}\end{array}$ & 2 \\
\hline IFR & $\begin{array}{l}\text { Airspace } \\
\text { Boundaries }\end{array}$ & $\begin{array}{l}\text { Intersection, Waypoint, } \\
\text { or Fix Symbol }\end{array}$ & $\begin{array}{c}\text { Yes } \\
x^{2}(1, \mathrm{~N}=105)= \\
105.00^{*}\end{array}$ & $\begin{array}{c}\text { Yes } \\
x^{2}(1, \mathrm{~N}= \\
105)=19.29 *\end{array}$ & & 1 \\
\hline IFR & $\begin{array}{l}\text { Airspace } \\
\text { Boundaries }\end{array}$ & $\begin{array}{l}\text { Intersection, Waypoint, } \\
\text { or Fix ID of VOR, } \\
\text { FREQ, MAG BRG }\end{array}$ & $\begin{array}{c}\text { Yes } \\
x^{2}(1, \mathrm{~N}=105)= \\
101.04^{*}\end{array}$ & $\begin{array}{c}\text { Yes } \\
x^{2}(1, \mathrm{~N}= \\
104)=4.65^{*}\end{array}$ & & 1 \\
\hline IFR & $\begin{array}{l}\text { Airspace } \\
\text { Boundaries }\end{array}$ & $\begin{array}{l}\text { Intersection, Waypoint, } \\
\text { or Fix Coordinates }\end{array}$ & $\begin{array}{c}\text { Yes } \\
x^{2}(1, \mathrm{~N}=106)= \\
76.42^{*}\end{array}$ & $\begin{array}{c}\text { No } \\
x^{2}(1, \mathrm{~N}=98) \\
=41.80^{*}\end{array}$ & $\begin{array}{c}\text { No preference } \\
x^{2}(1, \mathrm{~N}=81) \\
=2.09\end{array}$ & 3 \\
\hline IFR & $\begin{array}{l}\text { Airspace } \\
\text { Boundaries }\end{array}$ & $\begin{array}{l}\text { Indication of MET } \\
\text { Report Required }\end{array}$ & $\begin{array}{c}\text { Yes } \\
x^{2}(1, \mathrm{~N}=91)= \\
65.15^{*}\end{array}$ & $\begin{array}{c}\text { No } \\
x^{2}(1, \mathrm{~N}=84) \\
=3.86^{*}\end{array}$ & $\begin{array}{c}\text { Yes } \\
x^{2}(1, \mathrm{~N}=51) \\
=5.67^{*}\end{array}$ & 2 \\
\hline IFR & $\begin{array}{l}\text { Airspace } \\
\text { Boundaries }\end{array}$ & $\begin{array}{l}\text { Minimum Reception } \\
\text { Altitude (MRA) }\end{array}$ & $\begin{array}{c}\text { Yes } \\
x^{2}(1, \mathrm{~N}=106)= \\
98.15^{*}\end{array}$ & $\begin{array}{c}\text { No } \\
x^{2}(1, \mathrm{~N}= \\
104)=16.96^{*}\end{array}$ & $\begin{array}{c}\text { Yes } \\
x^{2}(1, \mathrm{~N}=73) \\
=14.92^{*}\end{array}$ & 2 \\
\hline IFR & $\begin{array}{l}\text { Airspace } \\
\text { Boundaries }\end{array}$ & $\begin{array}{l}\text { Computer Navigation } \\
\text { Fix (CNF) and ID }\end{array}$ & $\begin{array}{c}\text { Yes } \\
x^{2}(1, \mathrm{~N}=92)= \\
84.17^{*}\end{array}$ & $\begin{array}{c}\text { No } \\
x^{2}(1, \mathrm{~N}=90) \\
=17.78^{*}\end{array}$ & $\begin{array}{c}\text { Yes } \\
x^{2}(1, \mathrm{~N}=65) \\
=16.75^{*}\end{array}$ & 2 \\
\hline IFR & $\begin{array}{l}\text { Airspace } \\
\text { Boundaries }\end{array}$ & Procedural Data Notes & $\begin{array}{c}\text { Yes } \\
x^{2}(1, \mathrm{~N}=101)= \\
93.16^{*}\end{array}$ & $\begin{array}{c}\text { No } \\
x^{2}(1, \mathrm{~N}=99) \\
=83.65^{*}\end{array}$ & $\begin{array}{c}\text { Yes } \\
x^{2}(1, \mathrm{~N}=95) \\
=6.58^{*}\end{array}$ & 2 \\
\hline
\end{tabular}




\begin{tabular}{|c|c|c|c|c|c|c|}
\hline $\begin{array}{l}\text { Chart } \\
\text { Type }\end{array}$ & $\begin{array}{l}\text { Information } \\
\text { Category }\end{array}$ & Information Element & $\begin{array}{l}\text { Q1: Should it be } \\
\text { displayed to } \\
\text { execute } \\
\text { procedure? }\end{array}$ & $\begin{array}{l}\text { Q2: Displayed } \\
\text { at all times? }\end{array}$ & $\begin{array}{l}\text { Q3: Displayed } \\
\text { Initially? }\end{array}$ & $\begin{array}{l}\text { Importance } \\
\text { Level }\end{array}$ \\
\hline IFR & Airways & $\begin{array}{l}\text { Airway Symbol (center } \\
\text { line) }\end{array}$ & $\begin{array}{c}\text { Yes } \\
x^{2}(1, \mathrm{~N}=105)= \\
105.00^{*}\end{array}$ & $\begin{array}{c}\text { Yes } \\
x^{2}(1, \mathrm{~N}= \\
105)=45.34^{*}\end{array}$ & & 1 \\
\hline IFR & Airways & Airway Magnetic Course & $\begin{array}{c}\text { Yes } \\
x^{2}(1, \mathrm{~N}=106)= \\
98.15^{*}\end{array}$ & $\begin{array}{c}\text { Yes } \\
x^{2}(1, \mathrm{~N}= \\
104)=18.62 *\end{array}$ & & 1 \\
\hline IFR & Airways & $\begin{array}{l}\text { Times of one-way } \\
\text { direction }\end{array}$ & $\begin{array}{c}\text { Yes } \\
x^{2}(1, \mathrm{~N}=105)= \\
97.15^{*}\end{array}$ & $\begin{array}{c}\text { No } \\
x^{2}(1, \mathrm{~N}= \\
103)=17.95^{*}\end{array}$ & $\begin{array}{c}\text { Yes } \\
x^{2}(1, \mathrm{~N}=73) \\
=30.26^{*}\end{array}$ & 2 \\
\hline IFR & Airways & $\begin{array}{l}\text { Total Distance Between } \\
\text { Navaids }\end{array}$ & $\begin{array}{c}\text { Yes } \\
x^{2}(1, \mathrm{~N}=106)= \\
102.04^{*}\end{array}$ & $\begin{array}{c}\text { No preference } \\
x^{2}(1, \mathrm{~N}= \\
105)=0.01\end{array}$ & & $\begin{array}{l}\text { Not Yet } \\
\text { Determined }\end{array}$ \\
\hline IFR & Airways & $\begin{array}{l}\text { Segment Minimum } \\
\text { Cruising Level or MEA }\end{array}$ & $\begin{array}{c}\text { Yes } \\
x^{2}(1, \mathrm{~N}=105)= \\
105.00^{*}\end{array}$ & $\begin{array}{c}\text { No preference } \\
x^{2}(1, N= \\
105)=3.44\end{array}$ & & 1 \\
\hline IFR & Airways & Segment MOCA & $\begin{array}{c}\text { Yes } \\
x^{2}(1, \mathrm{~N}=106)= \\
106.00^{*}\end{array}$ & $\begin{array}{c}\text { No preference } \\
x^{2}(1, N= \\
106)=0.94\end{array}$ & & $\begin{array}{l}\text { Not Yet } \\
\text { Determined }\end{array}$ \\
\hline IFR & Airways & Holding Patterns & $\begin{array}{c}\text { Yes } \\
x^{2}(1, \mathrm{~N}=106)= \\
102.04^{*}\end{array}$ & $\begin{array}{c}\text { No preference } \\
x^{2}(1, \mathrm{~N}= \\
105)=1.15 \\
\end{array}$ & & 1 \\
\hline IFR & Airways & $\begin{array}{l}\text { VOR Change Over Point } \\
\text { with Distances }\end{array}$ & $\begin{array}{c}\text { Yes } \\
x^{2}(1, \mathrm{~N}=105)= \\
89.61^{*}\end{array}$ & $\begin{array}{c}\text { No preference } \\
x^{2}(1, \mathrm{~N}= \\
101)=0.09\end{array}$ & & $\begin{array}{l}\text { Not Yet } \\
\text { Determined }\end{array}$ \\
\hline IFR & Airways & $\begin{array}{l}\text { Intersection, Waypoint, } \\
\text { or Fix Name }\end{array}$ & $\begin{array}{c}\text { Yes } \\
x^{2}(1, \mathrm{~N}=105)= \\
105.00^{*}\end{array}$ & $\begin{array}{c}\text { Yes } \\
x^{2}(1, \mathrm{~N}= \\
105)=22.87 *\end{array}$ & & 1 \\
\hline IFR & Airways & $\begin{array}{l}\text { Intersection, Waypoint, } \\
\text { or Fix Distance from } \\
\text { Reference DME }\end{array}$ & $\begin{array}{c}\text { Yes } \\
x^{2}(1, \mathrm{~N}=106)= \\
106.00^{*}\end{array}$ & $\begin{array}{c}\text { No preference } \\
x^{2}(1, \mathrm{~N}= \\
106)=3.77\end{array}$ & & 1 \\
\hline IFR & Airways & $\begin{array}{l}\text { Indication of compulsory } \\
\text { reporting }\end{array}$ & $\begin{array}{c}\text { Yes } \\
x^{2}(1, \mathrm{~N}=104)= \\
100.04^{*}\end{array}$ & $\begin{array}{c}\text { Yes } \\
x^{2}(1, \mathrm{~N}= \\
103)=31.54^{*}\end{array}$ & & 1 \\
\hline IFR & Airways & $\begin{array}{l}\text { Fix Formation bearing, } \\
\text { frequency, ID of Remote } \\
\text { Navaid }\end{array}$ & $\begin{array}{c}\text { Yes } \\
x^{2}(1, \mathrm{~N}=100)= \\
96.04^{*}\end{array}$ & $\begin{array}{c}\text { No } \\
x^{2}(1, \mathrm{~N}=99) \\
=6.31^{*}\end{array}$ & $\begin{array}{c}\text { Yes } \\
x^{2}(1, \mathrm{~N}=62) \\
=31.23^{*}\end{array}$ & 2 \\
\hline IFR & Airways & $\begin{array}{l}\text { Minimum Crossing } \\
\text { Altitude (MCA) }\end{array}$ & $\begin{array}{c}\text { Yes } \\
x^{2}(1, N=105)= \\
97.15^{*}\end{array}$ & $\begin{array}{c}\text { Yes } \\
x^{2}(1, \mathrm{~N}= \\
103)=9.33^{*}\end{array}$ & & 1 \\
\hline IFR & Airways & Transition Text & $\begin{array}{c}\text { Yes } \\
x^{2}(1, \mathrm{~N}=96)= \\
84.38^{*}\end{array}$ & $\begin{array}{c}\text { No } \\
x^{2}(1, \mathrm{~N}=93) \\
=63.75^{*}\end{array}$ & $\begin{array}{c}\text { Yes } \\
x^{2}(1, \mathrm{~N}=85) \\
=12.81^{*}\end{array}$ & 2 \\
\hline
\end{tabular}




\begin{tabular}{|c|c|c|c|c|c|c|}
\hline $\begin{array}{l}\text { Chart } \\
\text { Type }\end{array}$ & $\begin{array}{l}\text { Information } \\
\text { Category }\end{array}$ & Information Element & $\begin{array}{l}\text { Q1: Should it be } \\
\text { displayed to } \\
\text { execute } \\
\text { procedure? }\end{array}$ & $\begin{array}{l}\text { Q2: Displayed } \\
\text { at all times? }\end{array}$ & $\begin{array}{l}\text { Q3: Displayed } \\
\text { Initially? }\end{array}$ & $\begin{array}{l}\text { Importance } \\
\text { Level }\end{array}$ \\
\hline IFR & Airways & General Notes & $\begin{array}{c}\text { Yes } \\
x^{2}(1, \mathrm{~N}=103)= \\
87.62^{*}\end{array}$ & $\begin{array}{c}\text { No } \\
x^{2}(1, \mathrm{~N}=99) \\
=87.36^{*}\end{array}$ & $\begin{array}{c}\text { No preference } \\
x^{2}(1, N=96) \\
=2.67\end{array}$ & 2 \\
\hline IFR & Navigation Aids & Navaid Symbol & $\begin{array}{c}\text { Yes } \\
x^{2}(1, \mathrm{~N}=106)= \\
106.00^{*}\end{array}$ & $\begin{array}{c}\text { Yes } \\
x^{2}(1, \mathrm{~N}= \\
106)=36.26^{*}\end{array}$ & & 1 \\
\hline IFR & Navigation Aids & Navaid Name & $\begin{array}{c}\text { Yes } \\
x^{2}(1, \mathrm{~N}=106)= \\
106.00^{*}\end{array}$ & $\begin{array}{c}\text { Yes } \\
x^{2}(1, \mathrm{~N}= \\
106)=4.57^{*}\end{array}$ & & 1 \\
\hline IFR & Navigation Aids & Navaid Identifier & $\begin{array}{c}\text { Yes } \\
x^{2}(1, \mathrm{~N}=106)= \\
106.00^{*}\end{array}$ & $\begin{array}{c}\text { No preference } \\
x^{2}(1, \mathrm{~N}= \\
106)=3.77 \\
\end{array}$ & & 1 \\
\hline IFR & Navigation Aids & Navaid Frequency & $\begin{array}{c}\text { Yes } \\
x^{2}(1, \mathrm{~N}=106)= \\
102.04^{*}\end{array}$ & $\begin{array}{c}\text { No preference } \\
x^{2}(1, N= \\
105)=2.75\end{array}$ & & 1 \\
\hline IFR & Navigation Aids & Navaid Coordinates & $\begin{array}{c}\text { Yes } \\
x^{2}(1, \mathrm{~N}=104)= \\
58.50^{*}\end{array}$ & $\begin{array}{c}\text { No } \\
x^{2}(1, \mathrm{~N}=91) \\
=58.56^{*}\end{array}$ & $\begin{array}{c}\text { No preference } \\
x^{2}(1, \mathrm{~N}=82) \\
=2.39\end{array}$ & 3 \\
\hline IFR & Navigation Aids & $\begin{array}{l}\text { Navaid Class (e.g., H, T, } \\
\text { and L) }\end{array}$ & $\begin{array}{c}\text { Yes } \\
x^{2}(1, \mathrm{~N}=105)= \\
59.44^{*}\end{array}$ & $\begin{array}{c}\text { No } \\
x^{2}(1, \mathrm{~N}=92) \\
=69.57^{*}\end{array}$ & $\begin{array}{l}\text { No preference } \\
\begin{array}{c}x^{2}(1, \mathrm{~N}=86) \\
=2.28\end{array}\end{array}$ & 3 \\
\hline IFR & Navigation Aids & $\begin{array}{l}\text { Navaid Station } \\
\text { Declination }\end{array}$ & $\begin{array}{c}\text { Yes } \\
x^{2}(1, \mathrm{~N}=97)= \\
54.94^{*}\end{array}$ & $\begin{array}{c}\text { No } \\
x^{2}(1, \mathrm{~N}=85) \\
=81.05^{*}\end{array}$ & $\begin{array}{c}\text { No } \\
x^{2}(1, \mathrm{~N}=84) \\
=3.86^{*}\end{array}$ & 3 \\
\hline IFR & Navigation Aids & DME Antenna Elevation & $\begin{array}{c}\text { Yes } \\
x^{2}(1, \mathrm{~N}=102)= \\
20.75^{*}\end{array}$ & $\begin{array}{c}\text { No } \\
x^{2}(1, \mathrm{~N}=74) \\
=66.22^{*}\end{array}$ & $\begin{array}{c}\text { No } \\
x^{2}(1, \mathrm{~N}=72) \\
=9.39 *\end{array}$ & 3 \\
\hline IFR & Navigation Aids & $\begin{array}{l}\text { Indication of True North } \\
\text { Navaids }\end{array}$ & $\begin{array}{c}\text { Yes } \\
x^{2}(1, \mathrm{~N}=103)= \\
54.61^{*}\end{array}$ & $\begin{array}{c}\text { No } \\
x^{2}(1, \mathrm{~N}=89) \\
=36.51^{*}\end{array}$ & $\begin{array}{l}\text { No preference } \\
\begin{array}{c}x^{2}(1, \mathrm{~N}=73) \\
=0.12\end{array}\end{array}$ & 3 \\
\hline IFR & Navigation Aids & $\begin{array}{l}\text { Notes on Navaid } \\
\text { Operational Status }\end{array}$ & $\begin{array}{c}\text { Yes } \\
x^{2}(1, \mathrm{~N}=106)= \\
79.85^{*}\end{array}$ & $\begin{array}{c}\text { No } \\
x^{2}(1, \mathrm{~N}=99) \\
=87.36^{*}\end{array}$ & $\begin{array}{l}\text { No preference } \\
\begin{array}{c}x^{2}(1, \mathrm{~N}=96) \\
=0.38\end{array}\end{array}$ & 2 \\
\hline IFR & Navigation Aids & $\begin{array}{l}\text { Broadcast Stations or } \\
\text { Marine Beacons }\end{array}$ & $\begin{array}{c}\text { Yes } \\
x^{2}(1, N=101)= \\
47.14^{*}\end{array}$ & $\begin{array}{c}\text { No } \\
x^{2}(1, \mathrm{~N}=85) \\
=81.05^{*}\end{array}$ & $\begin{array}{c}\text { No } \\
x^{2}(1, \mathrm{~N}=84) \\
=5.76^{*}\end{array}$ & 3 \\
\hline IFR & $\begin{array}{l}\text { Airport } \\
\text { Information }\end{array}$ & $\begin{array}{l}\text { Airport Symbol if for } \\
\text { IFR use (includes } \\
\text { suitable symbol type) }\end{array}$ & $\begin{array}{c}\text { Yes } \\
x^{2}(1, \mathrm{~N}=106)= \\
106.00^{*}\end{array}$ & $\begin{array}{c}\text { Yes } \\
x^{2}(1, N= \\
106)=8.49^{*}\end{array}$ & & 1 \\
\hline IFR & $\begin{array}{l}\text { Airport } \\
\text { Information }\end{array}$ & $\begin{array}{l}\text { Airport Identifier if for } \\
\text { IFR use }\end{array}$ & $\begin{array}{c}\text { Yes } \\
x^{2}(1, \mathrm{~N}=106)= \\
106.00^{*}\end{array}$ & $\begin{array}{c}\text { Yes } \\
x^{2}(1, N= \\
106)=8.49 *\end{array}$ & & 1 \\
\hline
\end{tabular}




\begin{tabular}{|c|c|c|c|c|c|c|}
\hline $\begin{array}{l}\text { Chart } \\
\text { Type }\end{array}$ & $\begin{array}{l}\text { Information } \\
\text { Category }\end{array}$ & Information Element & $\begin{array}{l}\text { Q1: Should it be } \\
\text { displayed to } \\
\text { execute } \\
\text { procedure? }\end{array}$ & $\begin{array}{l}\text { Q2: Displayed } \\
\text { at all times? }\end{array}$ & $\begin{array}{l}\text { Q3: Displayed } \\
\text { Initially? }\end{array}$ & $\begin{array}{l}\text { Importance } \\
\text { Level }\end{array}$ \\
\hline IFR & $\begin{array}{l}\text { Airport } \\
\text { Information }\end{array}$ & $\begin{array}{l}\text { Airport Attributes if for } \\
\text { IFR use }\end{array}$ & $\begin{array}{c}\text { Yes } \\
x^{2}(1, \mathrm{~N}=106)= \\
106.00^{*}\end{array}$ & $\begin{array}{c}\text { No } \\
x^{2}(1, \mathrm{~N}= \\
106)=18.26^{*}\end{array}$ & $\begin{array}{c}\text { Yes } \\
x^{2}(1, \mathrm{~N}=75) \\
=7.05^{*}\end{array}$ & 2 \\
\hline IFR & $\begin{array}{l}\text { Airport } \\
\text { Information }\end{array}$ & $\begin{array}{l}\text { Airport Symbol if for } \\
\text { VFR use (includes } \\
\text { suitable symbol type) }\end{array}$ & $\begin{array}{c}\text { Yes } \\
x^{2}(1, \mathrm{~N}=105)= \\
85.95^{*}\end{array}$ & $\begin{array}{c}\text { No } \\
x^{2}(1, \mathrm{~N}= \\
100)=23.04^{*}\end{array}$ & $\begin{array}{l}\text { No preference } \\
x^{2}(1, N=74) \\
\quad=1.35\end{array}$ & 2 \\
\hline IFR & $\begin{array}{l}\text { Airport } \\
\text { Information }\end{array}$ & $\begin{array}{l}\text { Airport Identifier if for } \\
\text { VFR use }\end{array}$ & $\begin{array}{c}\text { Yes } \\
x^{2}(1, \mathrm{~N}=104)= \\
88.62^{*}\end{array}$ & $\begin{array}{c}\text { No } \\
x^{2}(1, \mathrm{~N}= \\
100)=25.00^{*}\end{array}$ & $\begin{array}{l}\text { No preference } \\
x^{2}(1, \mathrm{~N}=75) \\
=1.08\end{array}$ & 2 \\
\hline IFR & $\begin{array}{l}\text { Airspace } \\
\text { Boundaries }\end{array}$ & $\begin{array}{l}\text { Unit Providing Approach } \\
\text { Control Service }\end{array}$ & $\begin{array}{c}\text { Yes } \\
x^{2}(1, \mathrm{~N}=105)= \\
97.15^{*}\end{array}$ & $\begin{array}{c}\text { No } \\
x^{2}(1, \mathrm{~N}= \\
103)=17.95^{*}\end{array}$ & $\begin{array}{c}\text { Yes } \\
x^{2}(1, \mathrm{~N}=73) \\
=18.75^{*}\end{array}$ & 2 \\
\hline IFR & $\begin{array}{l}\text { Airspace } \\
\text { Boundaries }\end{array}$ & $\begin{array}{l}\text { Airspace Class } \\
\text { Boundaries }\end{array}$ & $\begin{array}{c}\text { Yes } \\
x^{2}(1, \mathrm{~N}=106)= \\
94.34^{*}\end{array}$ & $\begin{array}{c}\text { No preference } \\
x^{2}(1, \mathrm{~N}= \\
103)=1.17\end{array}$ & & 1 \\
\hline IFR & $\begin{array}{l}\text { Airspace } \\
\text { Boundaries }\end{array}$ & Airspace Class Type & $\begin{array}{c}\text { Yes } \\
x^{2}(1, \mathrm{~N}=106)= \\
98.15^{*}\end{array}$ & $\begin{array}{c}\text { No preference } \\
x^{2}(1, N= \\
104)=1.88\end{array}$ & & $\begin{array}{l}\text { Not Yet } \\
\text { Determined }\end{array}$ \\
\hline IFR & $\begin{array}{l}\text { Airspace } \\
\text { Boundaries }\end{array}$ & $\begin{array}{l}\text { Airspace Class Name or } \\
\text { Call Sign }\end{array}$ & $\begin{array}{c}\text { Yes } \\
x^{2}(1, \mathrm{~N}=106)= \\
98.15^{*}\end{array}$ & $\begin{array}{c}\text { No } \\
x^{2}(1, \mathrm{~N}= \\
104)=7.54^{*}\end{array}$ & $\begin{array}{c}\text { Yes } \\
x^{2}(1, \mathrm{~N}=66) \\
=32.06^{*}\end{array}$ & 2 \\
\hline IFR & $\begin{array}{l}\text { Airspace } \\
\text { Boundaries }\end{array}$ & FIR/UIR Vertical Limits & $\begin{array}{c}\text { Yes } \\
x^{2}(1, \mathrm{~N}=101)= \\
93.16^{*}\end{array}$ & $\begin{array}{c}\text { No } \\
x^{2}(1, \mathrm{~N}=99) \\
=28.37^{*}\end{array}$ & $\begin{array}{c}\text { Yes } \\
x^{2}(1, \mathrm{~N}=76) \\
=23.21^{*}\end{array}$ & $\begin{array}{l}\text { Not Yet } \\
\text { Determined }\end{array}$ \\
\hline IFR & $\begin{array}{l}\text { Airspace } \\
\text { Boundaries }\end{array}$ & $\begin{array}{l}\text { Terminal Control Area } \\
\text { (TMA) Boundaries }\end{array}$ & $\begin{array}{c}\text { Yes } \\
x^{2}(1, \mathrm{~N}=105)= \\
97.15^{*}\end{array}$ & $\begin{array}{c}\text { No } \\
x^{2}(1, \mathrm{~N}= \\
103)=17.95^{*}\end{array}$ & $\begin{array}{c}\text { Yes } \\
x^{2}(1, \mathrm{~N}=73) \\
=11.52^{*}\end{array}$ & 2 \\
\hline IFR & Geography & $\begin{array}{l}\text { International Boundaries } \\
\text { (higher criticality where } \\
\text { appropriate) }\end{array}$ & $\begin{array}{c}\text { Yes } \\
x^{2}(1, \mathrm{~N}=103)= \\
95.16^{*}\end{array}$ & $\begin{array}{c}\text { No preference } \\
x^{2}(1, \mathrm{~N}= \\
101)=0.01\end{array}$ & & 1 \\
\hline IFR & Geography & $\begin{array}{l}\text { Terrain Contour } \\
\text { Elevations }\end{array}$ & $\begin{array}{c}\text { Yes } \\
x^{2}(1, \mathrm{~N}=106)= \\
86.94^{*}\end{array}$ & $\begin{array}{c}\text { No } \\
x^{2}(1, \mathrm{~N}= \\
101)=9.51^{*}\end{array}$ & $\begin{array}{c}\text { Yes } \\
x^{2}(1, \mathrm{~N}=66) \\
=17.52^{*}\end{array}$ & 2 \\
\hline IFR & Geography & Terrain Contours & $\begin{array}{c}\text { Yes } \\
x^{2}(1, \mathrm{~N}=104)= \\
88.62 *\end{array}$ & $\begin{array}{c}\text { No } \\
x^{2}(1, \mathrm{~N}= \\
100)=9.00^{*}\end{array}$ & $\begin{array}{c}\text { Yes } \\
x^{2}(1, \mathrm{~N}=65) \\
=16.75^{*}\end{array}$ & 2 \\
\hline IFR & Navigation Aids & Navaid Morse Code & $\begin{array}{c}\text { Yes } \\
x^{2}(1, \mathrm{~N}=105)= \\
59.44^{*}\end{array}$ & $\begin{array}{c}\text { No } \\
x^{2}(1, \mathrm{~N}=92) \\
=59.52^{*}\end{array}$ & $\begin{array}{c}\text { No } \\
x^{2}(1, \mathrm{~N}=83) \\
=5.31^{*}\end{array}$ & 2 \\
\hline
\end{tabular}




\begin{tabular}{|c|c|c|c|c|c|c|}
\hline $\begin{array}{l}\text { Chart } \\
\text { Type }\end{array}$ & $\begin{array}{l}\text { Information } \\
\text { Category }\end{array}$ & Information Element & $\begin{array}{l}\text { Q1: Should it be } \\
\text { displayed to } \\
\text { execute } \\
\text { procedure? }\end{array}$ & $\begin{array}{l}\text { Q2: Displayed } \\
\text { at all times? }\end{array}$ & $\begin{array}{l}\text { Q3: Displayed } \\
\text { Initially? }\end{array}$ & $\begin{array}{l}\text { Importance } \\
\text { Level }\end{array}$ \\
\hline IFR & $\begin{array}{l}\text { Airport } \\
\text { Information }\end{array}$ & $\begin{array}{l}\text { Airport Attributes if for } \\
\text { VFR use }\end{array}$ & $\begin{array}{c}\text { Yes } \\
x^{2}(1, \mathrm{~N}=104)= \\
67.85^{*}\end{array}$ & $\begin{array}{c}\text { No } \\
x^{2}(1, \mathrm{~N}=94) \\
=75.06^{*}\end{array}$ & $\begin{array}{l}\text { No preference } \\
x^{2}(1, \mathrm{~N}=89) \\
=1.90\end{array}$ & $\begin{array}{l}\text { Not Yet } \\
\text { Determined }\end{array}$ \\
\hline STAR & Identification & Revision Date & $\begin{array}{c}\text { Yes } \\
x^{2}(1, \mathrm{~N}=110)= \\
20.95^{*}\end{array}$ & $\begin{array}{c}\text { No } \\
x^{2}(1, \mathrm{~N}=79) \\
=50.24^{*}\end{array}$ & $\begin{array}{c}\text { Yes } \\
x^{2}(1, \mathrm{~N}=71) \\
=4.07^{*}\end{array}$ & 2 \\
\hline STAR & Identification & $\begin{array}{l}\text { Chart Index } \\
\text { Number/Page Number }\end{array}$ & $\begin{array}{c}\text { No preference } \\
x^{2}(1, N=108)= \\
3.00\end{array}$ & & & 4 \\
\hline STAR & Identification & Effective Date & $\begin{array}{c}\text { Yes } \\
x^{2}(1, \mathrm{~N}=110)= \\
42.04^{*}\end{array}$ & $\begin{array}{c}\text { No } \\
x^{2}(1, \mathrm{~N}=89) \\
=47.47^{*}\end{array}$ & $\begin{array}{c}\text { Yes } \\
x^{2}(1, \mathrm{~N}=77) \\
=10.92^{*}\end{array}$ & 2 \\
\hline STAR & Identification & City/Location Name & $\begin{array}{c}\text { Yes } \\
x^{2}(1, \mathrm{~N}=110)= \\
76.95^{*} \\
\end{array}$ & $\begin{array}{c}\text { No } \\
x^{2}(1, \mathrm{~N}= \\
101)=6.19^{*}\end{array}$ & $\begin{array}{c}\text { Yes } \\
x^{2}(1, \mathrm{~N}=63) \\
=21.73^{*} \\
\end{array}$ & 2 \\
\hline STAR & Identification & Airport Name & $\begin{array}{c}\text { Yes } \\
x^{2}(1, \mathrm{~N}=110)= \\
90.91^{*}\end{array}$ & $\begin{array}{c}\text { No preference } \\
x^{2}(1, N= \\
105)=1.15 \\
\end{array}$ & & $\begin{array}{l}\text { Not Yet } \\
\text { Determined }\end{array}$ \\
\hline STAR & Identification & Airport ICAO Identifier & $\begin{array}{c}\text { Yes } \\
x^{2}(1, \mathrm{~N}=110)= \\
102.15^{*}\end{array}$ & $\begin{array}{c}\text { Yes } \\
x^{2}(1, \mathrm{~N}= \\
108)=9.48^{*}\end{array}$ & & 1 \\
\hline STAR & Identification & $\begin{array}{l}\text { Procedure Name (e.g., } \\
\text { Canoga Eight) }\end{array}$ & $\begin{array}{c}\text { Yes } \\
x^{2}(1, \mathrm{~N}=110)= \\
110.00^{*}\end{array}$ & $\begin{array}{c}\text { Yes } \\
x^{2}(1, \mathrm{~N}= \\
110)=19.24^{*}\end{array}$ & & 1 \\
\hline STAR & Identification & $\begin{array}{l}\text { Procedure Identifier } \\
\text { (e.g., CNOG8.VNY) }\end{array}$ & $\begin{array}{c}\text { Yes } \\
x^{2}(1, \mathrm{~N}=110)= \\
106.04^{*}\end{array}$ & $\begin{array}{c}\text { No preference } \\
x^{2}(1, N= \\
109)=2.65 \\
\end{array}$ & & 1 \\
\hline STAR & Identification & Changes & $\begin{array}{c}\text { Yes } \\
x^{2}(1, \mathrm{~N}=100)= \\
40.96^{*}\end{array}$ & $\begin{array}{c}\text { No } \\
x^{2}(1, \mathrm{~N}=82) \\
=43.90^{*}\end{array}$ & $\begin{array}{c}\text { Yes } \\
x^{2}(1, \mathrm{~N}=71) \\
=10.27^{*}\end{array}$ & 2 \\
\hline STAR & Geography & Range & $\begin{array}{c}\text { Yes } \\
x^{2}(1, \mathrm{~N}=98)= \\
82.65^{*}\end{array}$ & $\begin{array}{l}\text { No preference } \\
x^{2}(1, N=94) \\
=0.04\end{array}$ & & 1 \\
\hline STAR & Geography & $\begin{array}{l}\text { Neat Lines (i.e., the lines } \\
\text { which separate the chart } \\
\text { from the margins) }\end{array}$ & $\begin{array}{c}\text { Yes } \\
x^{2}(1, \mathrm{~N}=105)= \\
24.77^{*}\end{array}$ & $\begin{array}{c}\text { No } \\
x^{2}(1, \mathrm{~N}=78) \\
=7.38^{*}\end{array}$ & $\begin{array}{l}\text { No preference } \\
x^{2}(1, \mathrm{~N}=51) \\
\quad=1.59\end{array}$ & $\begin{array}{l}\text { Not Yet } \\
\text { Determined }\end{array}$ \\
\hline STAR & Geography & $\begin{array}{l}\text { Parallels and Meridians } \\
\text { with AMAs, OROCAs, } \\
\text { MORAs }\end{array}$ & $\begin{array}{c}\text { Yes } \\
x^{2}(1, \mathrm{~N}=104)= \\
74.46^{*}\end{array}$ & $\begin{array}{c}\text { No } \\
x^{2}(1, \mathrm{~N}=96) \\
=18.38^{*}\end{array}$ & $\begin{array}{l}\text { No preference } \\
x^{2}(1, \mathrm{~N}=69) \\
=0.13\end{array}$ & 3 \\
\hline STAR & Geography & Parallels and Meridians & $\begin{array}{c}\text { Yes } \\
x^{2}(1, \mathrm{~N}=103)= \\
36.13^{*}\end{array}$ & $\begin{array}{c}\text { No } \\
x^{2}(1, \mathrm{~N}=82) \\
=46.88^{*}\end{array}$ & $\begin{array}{l}\text { No preference } \\
x^{2}(1, \mathrm{~N}=72) \\
=2.72\end{array}$ & 3 \\
\hline
\end{tabular}




\begin{tabular}{|c|c|c|c|c|c|c|}
\hline $\begin{array}{l}\text { Chart } \\
\text { Type }\end{array}$ & $\begin{array}{l}\text { Information } \\
\text { Category }\end{array}$ & Information Element & $\begin{array}{l}\text { Q1: Should it be } \\
\text { displayed to } \\
\text { execute } \\
\text { procedure? }\end{array}$ & $\begin{array}{l}\text { Q2: Displayed } \\
\text { at all times? }\end{array}$ & $\begin{array}{l}\text { Q3: Displayed } \\
\text { Initially? }\end{array}$ & $\begin{array}{l}\text { Importance } \\
\text { Level }\end{array}$ \\
\hline STAR & Geography & Water Features & $\begin{array}{c}\text { Yes } \\
x^{2}(1, \mathrm{~N}=110)= \\
61.13^{*}\end{array}$ & $\begin{array}{c}\text { No } \\
x^{2}(1, \mathrm{~N}=96) \\
=26.04^{*}\end{array}$ & $\begin{array}{l}\text { No preference } \\
x^{2}(1, \mathrm{~N}=73) \\
=0.67\end{array}$ & $\begin{array}{l}\text { Not Yet } \\
\text { Determined }\end{array}$ \\
\hline STAR & Geography & Cultural Features & $\begin{array}{c}\text { Yes } \\
x^{2}(1, \mathrm{~N}=106)= \\
29.58^{*}\end{array}$ & $\begin{array}{c}\text { No } \\
x^{2}(1, \mathrm{~N}=81) \\
=32.11^{*}\end{array}$ & $\begin{array}{l}\text { No preference } \\
x^{2}(1, N=66) \\
\quad=0.06\end{array}$ & $\begin{array}{l}\text { Not Yet } \\
\text { Determined }\end{array}$ \\
\hline STAR & Geography & Terrain Contours & $\begin{array}{c}\text { Yes } \\
x^{2}(1, \mathrm{~N}=109)= \\
101.15^{*} \\
\end{array}$ & $\begin{array}{c}\text { No preference } \\
x^{2}(1, \mathrm{~N}= \\
107)=2.10 \\
\end{array}$ & & 1 \\
\hline STAR & Geography & $\begin{array}{l}\text { Terrain Contour } \\
\text { Elevations }\end{array}$ & $\begin{array}{c}\text { Yes } \\
x^{2}(1, \mathrm{~N}=110)= \\
102.15^{*}\end{array}$ & $\begin{array}{c}\text { No preference } \\
x^{2}(1, \mathrm{~N}= \\
108)=1.33\end{array}$ & & $\begin{array}{l}\text { Not Yet } \\
\text { Determined }\end{array}$ \\
\hline STAR & Geography & Contour Interval Legend & $\begin{array}{c}\text { Yes } \\
x^{2}(1, \mathrm{~N}=108)= \\
85.33^{*}\end{array}$ & $\begin{array}{c}\text { No } \\
x^{2}(1, \mathrm{~N}= \\
102)=50.82^{*}\end{array}$ & $\begin{array}{l}\text { No preference } \\
x^{2}(1, N=87) \\
\quad=0.93\end{array}$ & 3 \\
\hline STAR & Geography & Spot Elevations & $\begin{array}{c}\text { Yes } \\
x^{2}(1, \mathrm{~N}=108)= \\
92.59^{*}\end{array}$ & $\begin{array}{c}\text { No preference } \\
x^{2}(1, \mathrm{~N}= \\
104)=0.04 \\
\end{array}$ & & 1 \\
\hline STAR & Geography & $\begin{array}{l}\text { Highest Reference Point } \\
\text { (within neat lines) }\end{array}$ & $\begin{array}{c}\text { Yes } \\
x^{2}(1, \mathrm{~N}=109)= \\
101.15^{*}\end{array}$ & $\begin{array}{c}\text { Yes } \\
x^{2}(1, \mathrm{~N}= \\
107)=4.12^{*}\end{array}$ & & 1 \\
\hline STAR & Geography & $\begin{array}{l}\text { International Boundaries } \\
\text { (higher criticality where } \\
\text { appropriate) }\end{array}$ & $\begin{array}{c}\text { Yes } \\
x^{2}(1, \mathrm{~N}=107)= \\
95.34^{*}\end{array}$ & $\begin{array}{c}\text { No } \\
x^{2}(1, \mathrm{~N}= \\
104)=7.54^{*}\end{array}$ & $\begin{array}{c}\text { Yes } \\
x^{2}(1, \mathrm{~N}=66) \\
=13.64^{*}\end{array}$ & $\begin{array}{l}\text { Not Yet } \\
\text { Determined }\end{array}$ \\
\hline STAR & Obstacle & $\begin{array}{l}\text { Obstacle Symbols and } \\
\text { Elevation }\end{array}$ & $\begin{array}{c}\text { Yes } \\
x^{2}(1, \mathrm{~N}=107)= \\
107.00^{*}\end{array}$ & $\begin{array}{c}\text { Yes } \\
x^{2}(1, \mathrm{~N}= \\
107)=7.86^{*}\end{array}$ & & 1 \\
\hline STAR & $\begin{array}{l}\text { Minimum } \\
\text { Area/Sector } \\
\text { Altitudes } \\
\end{array}$ & $\begin{array}{l}\text { MSA Reference } \\
\text { Point/Center }\end{array}$ & $\begin{array}{c}\text { Yes } \\
x^{2}(1, \mathrm{~N}=110)= \\
98.33^{*}\end{array}$ & $\begin{array}{c}\text { No preference } \\
x^{2}(1, \mathrm{~N}= \\
107)=0.23 \\
\end{array}$ & & $\begin{array}{l}\text { Not Yet } \\
\text { Determined }\end{array}$ \\
\hline STAR & $\begin{array}{l}\text { Minimum } \\
\text { Area/Sector } \\
\text { Altitudes }\end{array}$ & $\begin{array}{l}\text { MSA Distance when } \\
\text { other than 25nm }\end{array}$ & $\begin{array}{c}\text { Yes } \\
x^{2}(1, \mathrm{~N}=110)= \\
102.15^{*}\end{array}$ & $\begin{array}{c}\text { No preference } \\
x^{2}(1, N= \\
108)=2.37\end{array}$ & & $\begin{array}{l}\text { Not Yet } \\
\text { Determined }\end{array}$ \\
\hline STAR & $\begin{array}{l}\text { Minimum } \\
\text { Area/Sector } \\
\text { Altitudes } \\
\end{array}$ & MSA Sector Radials & $\begin{array}{c}\text { Yes } \\
x^{2}(1, \mathrm{~N}=109)= \\
109.00^{*}\end{array}$ & $\begin{array}{c}\text { No preference } \\
x^{2}(1, N= \\
109)=2.65 \\
\end{array}$ & & $\begin{array}{l}\text { Not Yet } \\
\text { Determined }\end{array}$ \\
\hline STAR & $\begin{array}{l}\text { Minimum } \\
\text { Area/Sector } \\
\text { Altitudes }\end{array}$ & $\begin{array}{l}\text { MSA Minimum } \\
\text { Altitudes }\end{array}$ & $\begin{array}{c}\text { Yes } \\
x^{2}(1, \mathrm{~N}=109)= \\
109.00^{*}\end{array}$ & $\begin{array}{c}\text { Yes } \\
x^{2}(1, \mathrm{~N}= \\
109)=5.73^{*}\end{array}$ & & 1 \\
\hline STAR & $\begin{array}{l}\text { Minimum } \\
\text { Area/Sector } \\
\text { Altitudes }\end{array}$ & $\begin{array}{l}\text { Minimum Radar } \\
\text { Altitudes and Sectors }\end{array}$ & $\begin{array}{c}\text { Yes } \\
x^{2}(1, \mathrm{~N}=110)= \\
110.00^{*}\end{array}$ & $\begin{array}{c}\text { No } \\
x^{2}(1, N= \\
110)=8.18^{*}\end{array}$ & $\begin{array}{c}\text { Yes } \\
x^{2}(1, \mathrm{~N}=70) \\
=4.63^{*}\end{array}$ & 2 \\
\hline
\end{tabular}




\begin{tabular}{|c|c|c|c|c|c|c|}
\hline $\begin{array}{l}\text { Chart } \\
\text { Type }\end{array}$ & $\begin{array}{l}\text { Information } \\
\text { Category }\end{array}$ & Information Element & $\begin{array}{l}\text { Q1: Should it be } \\
\text { displayed to } \\
\text { execute } \\
\text { procedure? }\end{array}$ & $\begin{array}{l}\text { Q2: Displayed } \\
\text { at all times? }\end{array}$ & $\begin{array}{l}\text { Q3: Displayed } \\
\text { Initially? }\end{array}$ & $\begin{array}{l}\text { Importance } \\
\text { Level }\end{array}$ \\
\hline STAR & $\begin{array}{l}\text { Minimum } \\
\text { Area/Sector } \\
\text { Altitudes }\end{array}$ & $\begin{array}{l}\text { AMA, OROCA, or grid } \\
\text { MORA where } \\
\text { established }\end{array}$ & $\begin{array}{c}\text { Yes } \\
x^{2}(1, \mathrm{~N}=104)= \\
88.62 *\end{array}$ & $\begin{array}{c}\text { No } \\
x^{2}(1, \mathrm{~N}= \\
100)=6.76^{*}\end{array}$ & $\begin{array}{l}\text { No preference } \\
x^{2}(1, N=63) \\
=1.92\end{array}$ & $\begin{array}{l}\text { Not Yet } \\
\text { Determined }\end{array}$ \\
\hline STAR & Navigation & FIR/UIR Boundaries & $\begin{array}{c}\text { Yes } \\
x^{2}(1, \mathrm{~N}=94)= \\
55.15^{*}\end{array}$ & $\begin{array}{c}\text { No } \\
x^{2}(1, \mathrm{~N}=83) \\
=33.84^{*}\end{array}$ & $\begin{array}{l}\text { No preference } \\
x^{2}(1, N=68) \\
\quad=0.53\end{array}$ & $\begin{array}{l}\text { Not Yet } \\
\text { Determined }\end{array}$ \\
\hline STAR & Navigation & $\begin{array}{l}\text { Prohibited, Restricted } \\
\text { and Danger Airspace } \\
\text { Graphic }\end{array}$ & $\begin{array}{c}\text { Yes } \\
x^{2}(1, \mathrm{~N}=110)= \\
110.00^{*}\end{array}$ & $\begin{array}{c}\text { Yes } \\
x^{2}(1, \mathrm{~N}= \\
110)=4.40^{*}\end{array}$ & & 1 \\
\hline STAR & Navigation & $\begin{array}{l}\text { Prohibited, Restricted } \\
\text { and Danger Airspace } \\
\text { Label }\end{array}$ & $\begin{array}{c}\text { Yes } \\
x^{2}(1, \mathrm{~N}=109)= \\
109.00^{*}\end{array}$ & $\begin{array}{c}\text { No } \\
x^{2}(1, \mathrm{~N}= \\
109)=5.73^{*}\end{array}$ & $\begin{array}{c}\text { Yes } \\
x^{2}(1, \mathrm{~N}=67) \\
=12.55^{*}\end{array}$ & $\begin{array}{l}\text { Not Yet } \\
\text { Determined }\end{array}$ \\
\hline STAR & Navigation & $\begin{array}{l}\text { Prohibited, Restricted } \\
\text { and Danger Airspace } \\
\text { Narrative }\end{array}$ & $\begin{array}{c}\text { Yes } \\
x^{2}(1, \mathrm{~N}=110)= \\
90.91^{*}\end{array}$ & $\begin{array}{c}\text { No } \\
x^{2}(1, \mathrm{~N}= \\
105)=40.24^{*}\end{array}$ & $\begin{array}{l}\text { No preference } \\
x^{2}(1, N=85) \\
\quad=0.95\end{array}$ & 2 \\
\hline STAR & Navigation & $\begin{array}{l}\text { Special Use Airspace - } \\
\text { Other }\end{array}$ & $\begin{array}{c}\text { Yes } \\
x^{2}(1, \mathrm{~N}=109)= \\
93.59^{*}\end{array}$ & $\begin{array}{c}\text { No } \\
x^{2}(1, \mathrm{~N}= \\
105)=26.75^{*}\end{array}$ & $\begin{array}{c}\text { Yes } \\
x^{2}(1, \mathrm{~N}=79) \\
=15.51^{*}\end{array}$ & 2 \\
\hline STAR & Navigation & Transition Level & $\begin{array}{c}\text { Yes } \\
x^{2}(1, \mathrm{~N}=104)= \\
81.38^{*}\end{array}$ & $\begin{array}{c}\text { No } \\
x^{2}(1, \mathrm{~N}=98) \\
=8.00^{*}\end{array}$ & $\begin{array}{c}\text { Yes } \\
x^{2}(1, \mathrm{~N}=63) \\
=21.73^{*}\end{array}$ & 2 \\
\hline STAR & $\begin{array}{l}\text { Airport } \\
\text { Information }\end{array}$ & $\begin{array}{l}\text { Primary Airport Shaded } \\
\text { Area }\end{array}$ & $\begin{array}{c}\text { Yes } \\
x^{2}(1, \mathrm{~N}=108)= \\
92.59^{*}\end{array}$ & $\begin{array}{c}\text { Yes } \\
x^{2}(1, \mathrm{~N}= \\
104)=6.50^{*}\end{array}$ & & 1 \\
\hline STAR & $\begin{array}{l}\text { Airport } \\
\text { Information }\end{array}$ & $\begin{array}{l}\text { Primary Airport } \\
\text { elevation }\end{array}$ & $\begin{array}{c}\text { Yes } \\
x^{2}(1, \mathrm{~N}=110)= \\
87.31^{*}\end{array}$ & $\begin{array}{c}\text { No preference } \\
x^{2}(1, N= \\
104)=3.12\end{array}$ & & 1 \\
\hline STAR & $\begin{array}{l}\text { Airport } \\
\text { Information }\end{array}$ & $\begin{array}{l}\text { Primary Airport Runway } \\
\text { Layout }\end{array}$ & $\begin{array}{c}\text { Yes } \\
x^{2}(1, \mathrm{~N}=110)= \\
98.33^{*}\end{array}$ & $\begin{array}{c}\text { Yes } \\
x^{2}(1, \mathrm{~N}= \\
107)=20.64^{*}\end{array}$ & & 1 \\
\hline STAR & $\begin{array}{l}\text { Airport } \\
\text { Information }\end{array}$ & Other Airport Symbols & $\begin{array}{c}\text { Yes } \\
x^{2}(1, \mathrm{~N}=108)= \\
88.93^{*}\end{array}$ & $\begin{array}{c}\text { No } \\
x^{2}(1, \mathrm{~N}= \\
103)=43.58^{*}\end{array}$ & $\begin{array}{c}\text { Yes } \\
x^{2}(1, \mathrm{~N}=85) \\
=14.41^{*} \\
\end{array}$ & 2 \\
\hline STAR & $\begin{array}{l}\text { Airport } \\
\text { Information }\end{array}$ & Other Airport Names & $\begin{array}{c}\text { Yes } \\
x^{2}(1, \mathrm{~N}=109)= \\
72.67^{*}\end{array}$ & $\begin{array}{c}\text { No } \\
x^{2}(1, \mathrm{~N}=99) \\
=53.83^{*}\end{array}$ & $\begin{array}{c}\text { Yes } \\
x^{2}(1, \mathrm{~N}=86) \\
=4.65^{*}\end{array}$ & 2 \\
\hline STAR & $\begin{array}{l}\text { Airport } \\
\text { Information }\end{array}$ & Other Airport Elevations & $\begin{array}{c}\text { Yes } \\
x^{2}(1, \mathrm{~N}=108)= \\
50.70^{*}\end{array}$ & $\begin{array}{c}\text { No } \\
x^{2}(1, \mathrm{~N}=91) \\
=61.81^{*}\end{array}$ & $\begin{array}{l}\text { No preference } \\
x^{2}(1, \mathrm{~N}=83) \\
=0.11\end{array}$ & 3 \\
\hline STAR & $\begin{array}{l}\text { Airport } \\
\text { Information }\end{array}$ & $\begin{array}{l}\text { Distances from last } \\
\text { STAR fix to airport }\end{array}$ & $\begin{array}{c}\text { Yes } \\
x^{2}(1, \mathrm{~N}=110)= \\
98.33^{*}\end{array}$ & $\begin{array}{c}\text { No preference } \\
x^{2}(1, N= \\
107)=0.46\end{array}$ & & $\begin{array}{l}\text { Not Yet } \\
\text { Determined }\end{array}$ \\
\hline
\end{tabular}




\begin{tabular}{|c|c|c|c|c|c|c|}
\hline $\begin{array}{l}\text { Chart } \\
\text { Type }\end{array}$ & $\begin{array}{l}\text { Information } \\
\text { Category }\end{array}$ & Information Element & $\begin{array}{l}\text { Q1: Should it be } \\
\text { displayed to } \\
\text { execute } \\
\text { procedure? }\end{array}$ & $\begin{array}{l}\text { Q2: Displayed } \\
\text { at all times? }\end{array}$ & $\begin{array}{l}\text { Q3: Displayed } \\
\text { Initially? }\end{array}$ & $\begin{array}{l}\text { Importance } \\
\text { Level }\end{array}$ \\
\hline STAR & $\begin{array}{l}\text { Navaid Used to } \\
\text { Form Leg of } \\
\text { Procedure }\end{array}$ & Navaid Symbol & $\begin{array}{c}\text { Yes } \\
x^{2}(1, \mathrm{~N}=109)= \\
109.00^{*}\end{array}$ & $\begin{array}{c}\text { Yes } \\
x^{2}(1, \mathrm{~N}= \\
109)=36.41^{*}\end{array}$ & & 1 \\
\hline STAR & $\begin{array}{l}\text { Navaid Used to } \\
\text { Form Leg of } \\
\text { Procedure }\end{array}$ & Navaid Name & $\begin{array}{c}\text { Yes } \\
x^{2}(1, \mathrm{~N}=110)= \\
110.00^{*}\end{array}$ & $\begin{array}{c}\text { Yes } \\
x^{2}(1, \mathrm{~N}= \\
110)=14.55^{*}\end{array}$ & & 1 \\
\hline STAR & $\begin{array}{l}\text { Navaid Used to } \\
\text { Form Leg of } \\
\text { Procedure }\end{array}$ & Navaid Identifier & $\begin{array}{c}\text { Yes } \\
x^{2}(1, \mathrm{~N}=109)= \\
105.04^{*}\end{array}$ & $\begin{array}{c}\text { Yes } \\
x^{2}(1, \mathrm{~N}= \\
108)=27.00^{*}\end{array}$ & & 1 \\
\hline STAR & $\begin{array}{l}\text { Navaid Used to } \\
\text { Form Leg of } \\
\text { Procedure }\end{array}$ & $\begin{array}{l}\text { Navaid } \\
\text { Frequency/Channel }\end{array}$ & $\begin{array}{c}\text { Yes } \\
x^{2}(1, N=110)= \\
106.04^{*}\end{array}$ & $\begin{array}{c}\text { Yes } \\
x^{2}(1, \mathrm{~N}= \\
109)=27.75^{*}\end{array}$ & & 1 \\
\hline STAR & $\begin{array}{l}\text { Navaid Used to } \\
\text { Form Leg of } \\
\text { Procedure }\end{array}$ & Navaid Morse Code & $\begin{array}{c}\text { Yes } \\
x^{2}(1, \mathrm{~N}=109)= \\
82.80^{*}\end{array}$ & $\begin{array}{c}\text { No } \\
x^{2}(1, \mathrm{~N}= \\
102)=50.82^{*}\end{array}$ & $\begin{array}{c}\text { Yes } \\
x^{2}(1, \mathrm{~N}=87) \\
=4.15^{*}\end{array}$ & 2 \\
\hline STAR & $\begin{array}{l}\text { Navaid Used to } \\
\text { Form Leg of } \\
\text { Procedure }\end{array}$ & $\begin{array}{l}\text { DME Availability (Text } \\
\text { information) }\end{array}$ & $\begin{array}{c}\text { Yes } \\
x^{2}(1, \mathrm{~N}=109)= \\
97.33^{*}\end{array}$ & $\begin{array}{c}\text { No } \\
x^{2}(1, \mathrm{~N}= \\
106)=15.09 *\end{array}$ & $\begin{array}{c}\text { Yes } \\
x^{2}(1, \mathrm{~N}=73) \\
=7.25^{*}\end{array}$ & 2 \\
\hline STAR & $\begin{array}{l}\text { Navaid Used to } \\
\text { Form Leg of } \\
\text { Procedure }\end{array}$ & Navaid Class & $\begin{array}{c}\text { Yes } \\
x^{2}(1, \mathrm{~N}=108)= \\
53.48^{*}\end{array}$ & $\begin{array}{c}\text { No } \\
x^{2}(1, \mathrm{~N}=92) \\
=69.57^{*}\end{array}$ & $\begin{array}{l}\text { No preference } \\
x^{2}(1, \mathrm{~N}=86) \\
=0.42\end{array}$ & 3 \\
\hline STAR & $\begin{array}{l}\text { Navaid Used to } \\
\text { Form Leg of } \\
\text { Procedure }\end{array}$ & $\begin{array}{l}\text { Navaid } \\
\text { Latitude/Longitude }\end{array}$ & $\begin{array}{c}\text { Yes } \\
x^{2}(1, \mathrm{~N}=109)= \\
22.03^{*}\end{array}$ & $\begin{array}{c}\text { No } \\
x^{2}(1, N=79) \\
=53.48^{*}\end{array}$ & $\begin{array}{c}\text { No } \\
x^{2}(1, \mathrm{~N}=72) \\
=10.89^{*}\end{array}$ & 3 \\
\hline STAR & $\begin{array}{l}\text { Navaid Used to } \\
\text { Form Fixes }\end{array}$ & Navaid Symbol & $\begin{array}{c}\text { Yes } \\
x^{2}(1, \mathrm{~N}=110)= \\
98.33^{*}\end{array}$ & $\begin{array}{c}\text { Yes } \\
x^{2}(1, \mathrm{~N}= \\
107)=17.28^{*}\end{array}$ & & 1 \\
\hline STAR & $\begin{array}{l}\text { Navaid Used to } \\
\text { Form Fixes }\end{array}$ & Navaid Name & $\begin{array}{c}\text { Yes } \\
x^{2}(1, \mathrm{~N}=110)= \\
98.33^{*}\end{array}$ & $\begin{array}{c}\text { Yes } \\
x^{2}(1, \mathrm{~N}= \\
107)=6.81^{*}\end{array}$ & & 1 \\
\hline STAR & $\begin{array}{l}\text { Navaid Used to } \\
\text { Form Fixes }\end{array}$ & Navaid Identifier & $\begin{array}{c}\text { Yes } \\
x^{2}(1, \mathrm{~N}=110)= \\
98.33^{*}\end{array}$ & $\begin{array}{c}\text { Yes } \\
x^{2}(1, \mathrm{~N}= \\
107)=30.36^{*}\end{array}$ & & 1 \\
\hline STAR & $\begin{array}{l}\text { Navaid Used to } \\
\text { Form Fixes }\end{array}$ & $\begin{array}{l}\text { Navaid } \\
\text { Frequency/Channel }\end{array}$ & $\begin{array}{c}\text { Yes } \\
x^{2}(1, N=109)= \\
101.15^{*}\end{array}$ & $\begin{array}{c}\text { Yes } \\
x^{2}(1, \mathrm{~N}= \\
107)=24.31^{*}\end{array}$ & & 1 \\
\hline STAR & $\begin{array}{l}\text { Navaid Used to } \\
\text { Form Fixes }\end{array}$ & Navaid Morse Code & $\begin{array}{c}\text { Yes } \\
x^{2}(1, \mathrm{~N}=110)= \\
70.40^{*}\end{array}$ & $\begin{array}{c}\text { No } \\
x^{2}(1, \mathrm{~N}=99) \\
=50.92^{*}\end{array}$ & $\begin{array}{c}\text { No preference } \\
x^{2}(1, \mathrm{~N}=85) \\
=2.65\end{array}$ & $\begin{array}{l}\text { Not Yet } \\
\text { Determined }\end{array}$ \\
\hline STAR & $\begin{array}{l}\text { Navaid Used to } \\
\text { Form Fixes }\end{array}$ & $\begin{array}{l}\text { DME Availability (Text } \\
\text { information) }\end{array}$ & $\begin{array}{c}\text { Yes } \\
x^{2}(1, \mathrm{~N}=109)= \\
79.35^{*}\end{array}$ & $\begin{array}{c}\text { No } \\
x^{2}(1, \mathrm{~N}= \\
101)=18.31 *\end{array}$ & $\begin{array}{c}\text { No preference } \\
x^{2}(1, N=72) \\
=0.89\end{array}$ & 2 \\
\hline STAR & $\begin{array}{l}\text { Navaid Used to } \\
\text { Form Fixes }\end{array}$ & Navaid Class & $\begin{array}{c}\text { Yes } \\
x^{2}(1, \mathrm{~N}=107)= \\
37.09^{*}\end{array}$ & $\begin{array}{c}\text { No } \\
x^{2}(1, \mathrm{~N}=85) \\
=52.81^{*}\end{array}$ & $\begin{array}{l}\text { No preference } \\
x^{2}(1, N=76) \\
=1.89\end{array}$ & 3 \\
\hline
\end{tabular}




\begin{tabular}{|c|c|c|c|c|c|c|}
\hline $\begin{array}{l}\text { Chart } \\
\text { Type }\end{array}$ & $\begin{array}{l}\text { Information } \\
\text { Category }\end{array}$ & Information Element & $\begin{array}{l}\text { Q1: Should it be } \\
\text { displayed to } \\
\text { execute } \\
\text { procedure? }\end{array}$ & $\begin{array}{l}\text { Q2: Displayed } \\
\text { at all times? }\end{array}$ & $\begin{array}{l}\text { Q3: Displayed } \\
\text { Initially? }\end{array}$ & $\begin{array}{l}\text { Importance } \\
\text { Level }\end{array}$ \\
\hline STAR & $\begin{array}{l}\text { Navaid Used to } \\
\text { Form Fixes }\end{array}$ & $\begin{array}{l}\text { Navaid } \\
\text { Latitude/Longitude }\end{array}$ & $\begin{array}{c}\text { Yes } \\
x^{2}(1, \mathrm{~N}=110)= \\
20.95^{*}\end{array}$ & $\begin{array}{c}\text { No } \\
x^{2}(1, \mathrm{~N}=79) \\
=53.48^{*}\end{array}$ & $\begin{array}{c}\text { No } \\
x^{2}(1, \mathrm{~N}=72) \\
=5.56^{*}\end{array}$ & 3 \\
\hline STAR & $\begin{array}{l}\text { Navaid Used to } \\
\text { Form Fixes }\end{array}$ & $\begin{array}{l}\text { Navaid Radials/Bearings } \\
\text { that form fixes }\end{array}$ & $\begin{array}{c}\text { Yes } \\
x^{2}(1, \mathrm{~N}=110)= \\
102.15^{*}\end{array}$ & $\begin{array}{c}\text { No preference } \\
x^{2}(1, N= \\
108)=1.81\end{array}$ & & 1 \\
\hline STAR & $\begin{array}{l}\text { Navaid Used to } \\
\text { Form Fixes }\end{array}$ & $\begin{array}{l}\text { DME Distances that } \\
\text { form fixes }\end{array}$ & $\begin{array}{c}\text { Yes } \\
x^{2}(1, \mathrm{~N}=108)= \\
104.04 *\end{array}$ & $\begin{array}{c}\text { Yes } \\
x^{2}(1, \mathrm{~N}= \\
107)=10.18^{*}\end{array}$ & & 1 \\
\hline STAR & $\begin{array}{l}\text { Instrument } \\
\text { Procedure } \\
\text { Courses/Tracks }\end{array}$ & $\begin{array}{l}\text { Symbol (e.g., line style, } \\
\text { etc.) }\end{array}$ & $\begin{array}{c}\text { Yes } \\
x^{2}(1, \mathrm{~N}=105)= \\
101.04^{*}\end{array}$ & $\begin{array}{c}\text { Yes } \\
x^{2}(1, \mathrm{~N}= \\
104)=36.96 *\end{array}$ & & 1 \\
\hline STAR & $\begin{array}{l}\text { Instrument } \\
\text { Procedure } \\
\text { Courses/Tracks }\end{array}$ & $\begin{array}{l}\text { Identifier (i.e., } \\
\text { CNOG8.AVE) }\end{array}$ & $\begin{array}{c}\text { Yes } \\
x^{2}(1, \mathrm{~N}=106)= \\
106.00^{*}\end{array}$ & $\begin{array}{c}\text { Yes } \\
x^{2}(1, \mathrm{~N}= \\
106)=8.49^{*}\end{array}$ & & 1 \\
\hline STAR & $\begin{array}{l}\text { Course } \\
\text { Definition }\end{array}$ & Heading & $\begin{array}{c}\text { Yes } \\
x^{2}(1, \mathrm{~N}=109)= \\
105.04^{*}\end{array}$ & $\begin{array}{c}\text { Yes } \\
x^{2}(1, \mathrm{~N}= \\
108)=59.26^{*}\end{array}$ & & 1 \\
\hline STAR & $\begin{array}{l}\text { Course } \\
\text { Definition }\end{array}$ & Track & $\begin{array}{c}\text { Yes } \\
x^{2}(1, \mathrm{~N}=108)= \\
108.00^{*}\end{array}$ & $\begin{array}{c}\text { Yes } \\
x^{2}(1, \mathrm{~N}= \\
108)=59.26^{*}\end{array}$ & & 1 \\
\hline STAR & $\begin{array}{l}\text { Course } \\
\text { Definition }\end{array}$ & Radial & $\begin{array}{c}\text { Yes } \\
x^{2}(1, \mathrm{~N}=109)= \\
109.00^{*}\end{array}$ & $\begin{array}{c}\text { Yes } \\
x^{2}(1, \mathrm{~N}= \\
109)=69.44^{*}\end{array}$ & & 1 \\
\hline STAR & $\begin{array}{l}\text { Course } \\
\text { Definition }\end{array}$ & Segment Mileages & $\begin{array}{c}\text { Yes } \\
x^{2}(1, \mathrm{~N}=110)= \\
106.04^{*}\end{array}$ & $\begin{array}{c}\text { Yes } \\
x^{2}(1, \mathrm{~N}= \\
109)=23.86^{*}\end{array}$ & & 1 \\
\hline STAR & $\begin{array}{l}\text { Course } \\
\text { Definition }\end{array}$ & MEA/MOCA & $\begin{array}{c}\text { Yes } \\
x^{2}(1, \mathrm{~N}=110)= \\
98.33^{*}\end{array}$ & $\begin{array}{c}\text { No preference } \\
x^{2}(1, \mathrm{~N}= \\
107)=0.76 \\
\end{array}$ & & 1 \\
\hline STAR & $\begin{array}{l}\text { Course } \\
\text { Definition }\end{array}$ & $\begin{array}{l}\text { VOR Change Over } \\
\text { Points }\end{array}$ & $\begin{array}{c}\text { Yes } \\
x^{2}(1, \mathrm{~N}=110)= \\
94.58^{*}\end{array}$ & $\begin{array}{c}\text { No preference } \\
x^{2}(1, N= \\
106)=0.04\end{array}$ & & $\begin{array}{l}\text { Not Yet } \\
\text { Determined }\end{array}$ \\
\hline STAR & Holding Pattern & $\begin{array}{l}\text { Holding Pattern } \\
\text { Depiction }\end{array}$ & $\begin{array}{c}\text { Yes } \\
x^{2}(1, \mathrm{~N}=110)= \\
110.00^{*}\end{array}$ & $\begin{array}{c}\text { Yes } \\
x^{2}(1, \mathrm{~N}= \\
110)=11.78^{*}\end{array}$ & & 1 \\
\hline STAR & Holding Pattern & Holding Pattern Courses & $\begin{array}{c}\text { Yes } \\
x^{2}(1, \mathrm{~N}=110)= \\
110.00^{*}\end{array}$ & $\begin{array}{c}\text { Yes } \\
x^{2}(1, \mathrm{~N}= \\
110)=6.15^{*}\end{array}$ & & 1 \\
\hline STAR & Holding Pattern & $\begin{array}{l}\text { Holding Pattern Leg } \\
\text { Length }\end{array}$ & $\begin{array}{c}\text { Yes } \\
x^{2}(1, \mathrm{~N}=110)= \\
110.00^{*}\end{array}$ & $\begin{array}{c}\text { No preference } \\
x^{2}(1, \mathrm{~N}= \\
110)=0.04\end{array}$ & & 1 \\
\hline STAR & Holding Pattern & Holding Pattern Time & $\begin{array}{c}\text { Yes } \\
x^{2}(1, \mathrm{~N}=110)= \\
102.15^{*}\end{array}$ & $\begin{array}{c}\text { No } \\
x^{2}(1, \mathrm{~N}= \\
108)=14.81^{*}\end{array}$ & $\begin{array}{c}\text { Yes } \\
x^{2}(1, \mathrm{~N}=74) \\
=10.59 *\end{array}$ & 2 \\
\hline
\end{tabular}




\begin{tabular}{|c|c|c|c|c|c|c|}
\hline $\begin{array}{l}\text { Chart } \\
\text { Type }\end{array}$ & $\begin{array}{l}\text { Information } \\
\text { Category }\end{array}$ & Information Element & $\begin{array}{l}\text { Q1: Should it be } \\
\text { displayed to } \\
\text { execute } \\
\text { procedure? }\end{array}$ & $\begin{array}{l}\text { Q2: Displayed } \\
\text { at all times? }\end{array}$ & $\begin{array}{l}\text { Q3: Displayed } \\
\text { Initially? }\end{array}$ & $\begin{array}{l}\text { Importance } \\
\text { Level }\end{array}$ \\
\hline STAR & Holding Pattern & Holding Pattern Speed & $\begin{array}{c}\text { Yes } \\
x^{2}(1, \mathrm{~N}=110)= \\
102.15^{*}\end{array}$ & $\begin{array}{c}\text { No } \\
x^{2}(1, \mathrm{~N}= \\
108)=25.04^{*}\end{array}$ & $\begin{array}{c}\text { Yes } \\
x^{2}(1, \mathrm{~N}=80) \\
=11.25^{*}\end{array}$ & 2 \\
\hline STAR & Holding Pattern & Holding Pattern Altitude & $\begin{array}{c}\text { Yes } \\
x^{2}(1, \mathrm{~N}=110)= \\
106.04^{*}\end{array}$ & $\begin{array}{c}\text { No preference } \\
x^{2}(1, \mathrm{~N}= \\
109)=1.55 \\
\end{array}$ & & 1 \\
\hline STAR & Transitions & $\begin{array}{l}\text { Transition Courses } \\
\text { Depiction }\end{array}$ & $\begin{array}{c}\text { Yes } \\
x^{2}(1, \mathrm{~N}=109)= \\
109.00^{*}\end{array}$ & $\begin{array}{c}\text { Yes } \\
x^{2}(1, \mathrm{~N}= \\
109)=9.99 *\end{array}$ & & 1 \\
\hline STAR & Transitions & Transition Name & $\begin{array}{c}\text { Yes } \\
x^{2}(1, \mathrm{~N}=109)= \\
109.00^{*}\end{array}$ & $\begin{array}{c}\text { No preference } \\
x^{2}(1, N= \\
109)=2.06\end{array}$ & & 1 \\
\hline STAR & Transitions & $\begin{array}{l}\text { Transition Courses } \\
\text { computer codes }\end{array}$ & $\begin{array}{c}\text { Yes } \\
x^{2}(1, \mathrm{~N}=97)= \\
78.03^{*}\end{array}$ & $\begin{array}{c}\text { No } \\
x^{2}(1, \mathrm{~N}=92) \\
=36.57^{*}\end{array}$ & $\begin{array}{c}\text { Yes } \\
x^{2}(1, \mathrm{~N}=75) \\
=11.21^{*}\end{array}$ & 2 \\
\hline STAR & Transitions & $\begin{array}{l}\text { Transition Course - } \\
\text { Magnetic Values }\end{array}$ & $\begin{array}{c}\text { Yes } \\
x^{2}(1, \mathrm{~N}=106)= \\
98.15^{*}\end{array}$ & $\begin{array}{c}\text { No preference } \\
x^{2}(1, \mathrm{~N}= \\
104)=0.04 \\
\end{array}$ & & $\begin{array}{l}\text { Not Yet } \\
\text { Determined }\end{array}$ \\
\hline STAR & Transitions & $\begin{array}{l}\text { Transition Courses - } \\
\text { MEAs, MOCAs }\end{array}$ & $\begin{array}{c}\text { Yes } \\
x^{2}(1, \mathrm{~N}=109)= \\
101.15^{*}\end{array}$ & $\begin{array}{c}\text { No preference } \\
x^{2}(1, N= \\
107)=1.58 \\
\end{array}$ & & $\begin{array}{l}\text { Not Yet } \\
\text { Determined }\end{array}$ \\
\hline STAR & Transitions & $\begin{array}{l}\text { Transition Courses - } \\
\text { segment milages }\end{array}$ & $\begin{array}{c}\text { Yes } \\
x^{2}(1, \mathrm{~N}=109)= \\
109.00^{*}\end{array}$ & $\begin{array}{c}\text { No preference } \\
x^{2}(1, N= \\
109)=0.01 \\
\end{array}$ & & $\begin{array}{l}\text { Not Yet } \\
\text { Determined }\end{array}$ \\
\hline STAR & Transitions & $\begin{array}{l}\text { Transition Course notes } \\
\text { (e.g., DME required) }\end{array}$ & $\begin{array}{c}\text { Yes } \\
x^{2}(1, \mathrm{~N}=109)= \\
101.15^{*}\end{array}$ & $\begin{array}{c}\text { No } \\
x^{2}(1, \mathrm{~N}= \\
107)=10.18^{*}\end{array}$ & $\begin{array}{c}\text { Yes } \\
x^{2}(1, \mathrm{~N}=70) \\
=18.51^{*}\end{array}$ & 2 \\
\hline STAR & Transitions & Transition Text & $\begin{array}{c}\text { Yes } \\
x^{2}(1, \mathrm{~N}=109)= \\
93.59^{*}\end{array}$ & $\begin{array}{c}\text { No } \\
x^{2}(1, \mathrm{~N}= \\
105)=22.87^{*}\end{array}$ & $\begin{array}{c}\text { Yes } \\
x^{2}(1, \mathrm{~N}=77) \\
=21.83^{*}\end{array}$ & 2 \\
\hline STAR & $\begin{array}{l}\text { Intersection/Fixe } \\
\text { s on Procedure }\end{array}$ & Symbol & $\begin{array}{c}\text { Yes } \\
x^{2}(1, \mathrm{~N}=110)= \\
110.00^{*}\end{array}$ & $\begin{array}{c}\text { Yes } \\
x^{2}(1, \mathrm{~N}= \\
110)=44.55^{*}\end{array}$ & & 1 \\
\hline STAR & $\begin{array}{l}\text { Intersection/Fixe } \\
\text { s on Procedure }\end{array}$ & Names & $\begin{array}{c}\text { Yes } \\
x^{2}(1, \mathrm{~N}=110)= \\
110.00^{*}\end{array}$ & $\begin{array}{c}\text { Yes } \\
x^{2}(1, \mathrm{~N}= \\
110)=24.58^{*}\end{array}$ & & 1 \\
\hline STAR & $\begin{array}{l}\text { Intersection/Fixe } \\
\text { s on Procedure }\end{array}$ & Identifier & $\begin{array}{c}\text { Yes } \\
x^{2}(1, \mathrm{~N}=108)= \\
108.00^{*}\end{array}$ & $\begin{array}{c}\text { Yes } \\
x^{2}(1, \mathrm{~N}= \\
108)=19.59^{*}\end{array}$ & & 1 \\
\hline STAR & $\begin{array}{l}\text { Intersection/Fixe } \\
\text { s on Procedure }\end{array}$ & Latitude/Longitudes & $\begin{array}{c}\text { Yes } \\
x^{2}(1, \mathrm{~N}=109)= \\
41.18^{*}\end{array}$ & $\begin{array}{c}\text { No } \\
x^{2}(1, \mathrm{~N}=88) \\
=49.50^{*}\end{array}$ & $\begin{array}{c}\text { No preference } \\
x^{2}(1, N=77) \\
=0.32\end{array}$ & 3 \\
\hline STAR & $\begin{array}{l}\text { Intersection/Fixe } \\
\text { s on Procedure }\end{array}$ & MRA & $\begin{array}{c}\text { Yes } \\
x^{2}(1, \mathrm{~N}=104)= \\
77.88^{*}\end{array}$ & $\begin{array}{c}\text { No } \\
x^{2}(1, \mathrm{~N}=97) \\
=22.77^{*}\end{array}$ & $\begin{array}{l}\text { No preference } \\
x^{2}(1, \mathrm{~N}=72) \\
\quad=2.72\end{array}$ & $\begin{array}{l}\text { Not Yet } \\
\text { Determined }\end{array}$ \\
\hline
\end{tabular}




\begin{tabular}{|c|c|c|c|c|c|c|}
\hline $\begin{array}{l}\text { Chart } \\
\text { Type }\end{array}$ & $\begin{array}{l}\text { Information } \\
\text { Category }\end{array}$ & Information Element & $\begin{array}{l}\text { Q1: Should it be } \\
\text { displayed to } \\
\text { execute } \\
\text { procedure? }\end{array}$ & $\begin{array}{l}\text { Q2: Displayed } \\
\text { at all times? }\end{array}$ & $\begin{array}{l}\text { Q3: Displayed } \\
\text { Initially? }\end{array}$ & $\begin{array}{l}\text { Importance } \\
\text { Level }\end{array}$ \\
\hline STAR & $\begin{array}{l}\text { Textual } \\
\text { Information }\end{array}$ & Runway arrival text & $\begin{array}{c}\text { Yes } \\
x^{2}(1, \mathrm{~N}=110)= \\
106.04^{*}\end{array}$ & $\begin{array}{c}\text { No preference } \\
x^{2}(1, N= \\
109)=3.31\end{array}$ & & 2 \\
\hline STAR & $\begin{array}{l}\text { Textual } \\
\text { Information }\end{array}$ & Transition Text & $\begin{array}{c}\text { Yes } \\
x^{2}(1, \mathrm{~N}=110)= \\
102.15^{*}\end{array}$ & $\begin{array}{c}\text { No } \\
x^{2}(1, \mathrm{~N}= \\
108)=9.48^{*}\end{array}$ & $\begin{array}{c}\text { Yes } \\
x^{2}(1, \mathrm{~N}=70) \\
=14.63^{*}\end{array}$ & 2 \\
\hline STAR & $\begin{array}{l}\text { Textual } \\
\text { Information }\end{array}$ & Notes & $\begin{array}{c}\text { Yes } \\
x^{2}(1, \mathrm{~N}=105)= \\
89.61^{*}\end{array}$ & $\begin{array}{c}\text { No } \\
x^{2}(1, \mathrm{~N}= \\
101)=64.96^{*}\end{array}$ & $\begin{array}{c}\text { Yes } \\
x^{2}(1, \mathrm{~N}=91) \\
=15.04^{*}\end{array}$ & 2 \\
\hline STAR & $\begin{array}{l}\text { Textual } \\
\text { Information }\end{array}$ & Noise Abatement & $\begin{array}{c}\text { Yes } \\
x^{2}(1, \mathrm{~N}=110)= \\
83.78^{*}\end{array}$ & $\begin{array}{c}\text { No } \\
x^{2}(1, \mathrm{~N}= \\
103)=63.70^{*}\end{array}$ & $\begin{array}{c}\text { Yes } \\
x^{2}(1, \mathrm{~N}=92) \\
=11.13^{*}\end{array}$ & 2 \\
\hline STAR & $\begin{array}{l}\text { Textual } \\
\text { Information }\end{array}$ & $\begin{array}{l}\text { Performance limitations } \\
\text { (e.g., bank limits) }\end{array}$ & $\begin{array}{c}\text { Yes } \\
x^{2}(1, \mathrm{~N}=109)= \\
97.33^{*}\end{array}$ & $\begin{array}{c}\text { No } \\
x^{2}(1, \mathrm{~N}= \\
106)=13.62^{*}\end{array}$ & $\begin{array}{c}\text { Yes } \\
x^{2}(1, \mathrm{~N}=72) \\
=8.00^{*}\end{array}$ & 2 \\
\hline STAR & $\begin{array}{l}\text { Textual } \\
\text { Information }\end{array}$ & Text-Only Procedures & $\begin{array}{c}\text { Yes } \\
x^{2}(1, \mathrm{~N}=109)= \\
97.33^{*}\end{array}$ & $\begin{array}{c}\text { No } \\
x^{2}(1, \mathrm{~N}= \\
106)=27.51 *\end{array}$ & $\begin{array}{c}\text { Yes } \\
x^{2}(1, \mathrm{~N}=80) \\
=7.20^{*}\end{array}$ & 2 \\
\hline STAR & $\begin{array}{l}\text { Textual } \\
\text { Information }\end{array}$ & General Notes & $\begin{array}{c}\text { Yes } \\
x^{2}(1, \mathrm{~N}=107)= \\
87.93^{*}\end{array}$ & $\begin{array}{c}\text { No } \\
x^{2}(1, \mathrm{~N}= \\
102)=72.51 *\end{array}$ & $\begin{array}{l}\text { No preference } \\
x^{2}(1, \mathrm{~N}=94) \\
=2.72\end{array}$ & 2 \\
\hline STAR & $\begin{array}{l}\text { Textual } \\
\text { Information }\end{array}$ & Procedural Data Notes & $\begin{array}{c}\text { Yes } \\
x^{2}(1, \mathrm{~N}=107)= \\
87.93^{*}\end{array}$ & $\begin{array}{c}\text { No } \\
x^{2}(1, \mathrm{~N}= \\
102)=35.29^{*}\end{array}$ & $\begin{array}{l}\text { No preference } \\
x^{2}(1, \mathrm{~N}=81) \\
=2.09\end{array}$ & 2 \\
\hline STAR & $\begin{array}{l}\text { Textual } \\
\text { Information }\end{array}$ & $\begin{array}{l}\text { Crossing Altitude } \\
\text { Restrictions }\end{array}$ & $\begin{array}{c}\text { Yes } \\
x^{2}(1, \mathrm{~N}=110)= \\
110.00^{*}\end{array}$ & $\begin{array}{c}\text { Yes } \\
x^{2}(1, \mathrm{~N}= \\
110)=32.73^{*}\end{array}$ & & 1 \\
\hline STAR & $\begin{array}{l}\text { Textual } \\
\text { Information }\end{array}$ & Speed restrictions & $\begin{array}{c}\text { Yes } \\
x^{2}(1, \mathrm{~N}=110)= \\
110.00^{*}\end{array}$ & $\begin{array}{c}\text { Yes } \\
x^{2}(1, \mathrm{~N}= \\
110)=22.73^{*}\end{array}$ & & 1 \\
\hline STAR & Communications & ATIS Arrival Frequency & $\begin{array}{c}\text { Yes } \\
x^{2}(1, \mathrm{~N}=110)= \\
106.04^{*}\end{array}$ & $\begin{array}{c}\text { No preference } \\
x^{2}(1, N= \\
109)=3.31\end{array}$ & & 2 \\
\hline STAR & Communications & $\begin{array}{l}\text { ACARS-D - ATIS, } \\
\text { TWIP }\end{array}$ & $\begin{array}{c}\text { Yes } \\
x^{2}(1, N=99)= \\
91.16^{*}\end{array}$ & $\begin{array}{c}\text { No } \\
x^{2}(1, \mathrm{~N}=97) \\
=12.63^{*}\end{array}$ & $\begin{array}{c}\text { Yes } \\
x^{2}(1, \mathrm{~N}=66) \\
=11.88^{*}\end{array}$ & 2 \\
\hline STAR & Communications & $\begin{array}{l}\text { Approach Control } \\
\text { (Arrival) }\end{array}$ & $\begin{array}{c}\text { Yes } \\
x^{2}(1, \mathrm{~N}=110)= \\
106.04^{*}\end{array}$ & $\begin{array}{c}\text { No preference } \\
x^{2}(1, \mathrm{~N}= \\
109)=0.74 \\
\end{array}$ & & $\begin{array}{l}\text { Not Yet } \\
\text { Determined }\end{array}$ \\
\hline STAR & Communications & $\begin{array}{l}\text { Communications } \\
\text { Boundaries }\end{array}$ & $\begin{array}{c}\text { Yes } \\
x^{2}(1, \mathrm{~N}=108)= \\
81.81^{*}\end{array}$ & $\begin{array}{c}\text { No } \\
x^{2}(1, \mathrm{~N}= \\
101)=41.83^{*}\end{array}$ & $\begin{array}{l}\text { No preference } \\
x^{2}(1, N=83) \\
=2.71\end{array}$ & $\begin{array}{l}\text { Not Yet } \\
\text { Determined }\end{array}$ \\
\hline STAR & Communications & Lost Comm Procedure & $\begin{array}{c}\text { Yes } \\
x^{2}(1, \mathrm{~N}=109)= \\
105.04^{*}\end{array}$ & $\begin{array}{c}\text { No } \\
x^{2}(1, \mathrm{~N}= \\
108)=31.15^{*}\end{array}$ & $\begin{array}{l}\text { No preference } \\
\begin{array}{c}x^{2}(1, N=83) \\
=0.01\end{array}\end{array}$ & 3 \\
\hline
\end{tabular}




\begin{tabular}{|c|c|c|c|c|c|c|}
\hline $\begin{array}{l}\text { Chart } \\
\text { Type }\end{array}$ & $\begin{array}{l}\text { Information } \\
\text { Category }\end{array}$ & Information Element & $\begin{array}{l}\text { Q1: Should it be } \\
\text { displayed to } \\
\text { execute } \\
\text { procedure? }\end{array}$ & $\begin{array}{l}\text { Q2: Displayed } \\
\text { at all times? }\end{array}$ & $\begin{array}{l}\text { Q3: Displayed } \\
\text { Initially? }\end{array}$ & $\begin{array}{l}\text { Importance } \\
\text { Level }\end{array}$ \\
\hline STAR & Communications & $\begin{array}{l}\text { Lost Comm Procedure } \\
\text { Outline Lines }\end{array}$ & $\begin{array}{c}\text { Yes } \\
x^{2}(1, \mathrm{~N}=109)= \\
101.15^{*}\end{array}$ & $\begin{array}{c}\text { No } \\
x^{2}(1, \mathrm{~N}= \\
107)=39.49 *\end{array}$ & $\begin{array}{l}\text { No preference } \\
x^{2}(1, \mathrm{~N}=86) \\
=0.19\end{array}$ & 2 \\
\hline STAR & Communications & $\begin{array}{l}\text { Transponder Setting } \\
\text { where appropriate }\end{array}$ & $\begin{array}{c}\text { Yes } \\
x^{2}(1, \mathrm{~N}=104)= \\
92.35^{*}\end{array}$ & $\begin{array}{c}\text { No } \\
x^{2}(1, \mathrm{~N}= \\
101)=23.77 *\end{array}$ & $\begin{array}{c}\text { No preference } \\
x^{2}(1, \mathrm{~N}=75) \\
=2.25\end{array}$ & 2 \\
\hline SID & Identification & Revision Date & $\begin{array}{c}\text { Yes } \\
x^{2}(1, \mathrm{~N}=110)= \\
39.60^{*}\end{array}$ & $\begin{array}{c}\text { No } \\
x^{2}(1, \mathrm{~N}=88) \\
=46.55^{*}\end{array}$ & $\begin{array}{c}\text { Yes } \\
x^{2}(1, \mathrm{~N}=76) \\
=8.89^{*}\end{array}$ & 2 \\
\hline SID & Identification & $\begin{array}{l}\text { Chart Index } \\
\text { Number/Page Number }\end{array}$ & $\begin{array}{c}\text { Yes } \\
x^{2}(1, \mathrm{~N}=110)= \\
32.73^{*}\end{array}$ & $\begin{array}{c}\text { No } \\
x^{2}(1, \mathrm{~N}=85) \\
=25.99^{*}\end{array}$ & $\begin{array}{c}\text { Yes } \\
x^{2}(1, \mathrm{~N}=66) \\
=6.06^{*}\end{array}$ & 2 \\
\hline SID & Identification & Effective Date & $\begin{array}{c}\text { Yes } \\
x^{2}(1, \mathrm{~N}=109)= \\
51.61^{*}\end{array}$ & $\begin{array}{c}\text { No } \\
x^{2}(1, \mathrm{~N}=92) \\
=34.09^{*}\end{array}$ & $\begin{array}{c}\text { Yes } \\
x^{2}(1, \mathrm{~N}=74) \\
=28.59 *\end{array}$ & 2 \\
\hline SID & Identification & City/Location Name & $\begin{array}{c}\text { Yes } \\
x^{2}(1, \mathrm{~N}=110)= \\
98.33^{*}\end{array}$ & $\begin{array}{c}\text { No preference } \\
x^{2}(1, \mathrm{~N}= \\
107)=0.08 \\
\end{array}$ & & $\begin{array}{l}\text { Not Yet } \\
\text { Determined }\end{array}$ \\
\hline SID & Identification & Airport Name & $\begin{array}{c}\text { Yes } \\
x^{2}(1, N=110)= \\
106.04^{*}\end{array}$ & $\begin{array}{c}\text { Yes } \\
x^{2}(1, \mathrm{~N}= \\
109)=11.24^{*}\end{array}$ & & 1 \\
\hline SID & Identification & Airport ICAO Identifier & $\begin{array}{c}\text { Yes } \\
x^{2}(1, \mathrm{~N}=110)= \\
106.04^{*}\end{array}$ & $\begin{array}{c}\text { Yes } \\
x^{2}(1, \mathrm{~N}= \\
109)=27.75^{*}\end{array}$ & & 1 \\
\hline SID & Identification & $\begin{array}{l}\text { Procedure Name (e.g., } \\
\text { Canoga Eight) }\end{array}$ & $\begin{array}{c}\text { Yes } \\
x^{2}(1, \mathrm{~N}=110)= \\
106.04^{*}\end{array}$ & $\begin{array}{c}\text { Yes } \\
x^{2}(1, \mathrm{~N}= \\
109)=18.58^{*}\end{array}$ & & 1 \\
\hline SID & Identification & $\begin{array}{l}\text { Procedure Identifier } \\
\text { (e.g., } \\
\text { CNOG8.VNY+A42) }\end{array}$ & $\begin{array}{c}\text { Yes } \\
x^{2}(1, \mathrm{~N}=110)= \\
102.15^{*}\end{array}$ & $\begin{array}{c}\text { No preference } \\
x^{2}(1, N= \\
108)=3.70\end{array}$ & & 1 \\
\hline SID & Identification & $\begin{array}{l}\text { DP Type (e.g., Pilot Nav, } \\
\text { Vector, Noise, Obstacle) }\end{array}$ & $\begin{array}{c}\text { Yes } \\
x^{2}(1, \mathrm{~N}=110)= \\
87.31^{*}\end{array}$ & $\begin{array}{c}\text { No } \\
x^{2}(1, \mathrm{~N}= \\
104)=7.54^{*}\end{array}$ & $\begin{array}{c}\text { Yes } \\
x^{2}(1, \mathrm{~N}=66) \\
=44.18^{*}\end{array}$ & 2 \\
\hline SID & Identification & Changes & $\begin{array}{c}\text { Yes } \\
x^{2}(1, \mathrm{~N}=106)= \\
57.40^{*}\end{array}$ & $\begin{array}{c}\text { No } \\
x^{2}(1, \mathrm{~N}=92) \\
=56.35^{*}\end{array}$ & $\begin{array}{c}\text { Yes } \\
x^{2}(1, \mathrm{~N}=82) \\
=4.88^{*}\end{array}$ & 2 \\
\hline SID & Geography & Range & $\begin{array}{c}\text { Yes } \\
x^{2}(1, \mathrm{~N}=101)= \\
78.43^{*}\end{array}$ & $\begin{array}{l}\text { No preference } \\
x^{2}(1, \mathrm{~N}=95) \\
=0.09\end{array}$ & & $\begin{array}{l}\text { Not Yet } \\
\text { Determined }\end{array}$ \\
\hline SID & Geography & $\begin{array}{l}\text { Neat Lines (i.e., the lines } \\
\text { which separate the chart } \\
\text { from the margins) }\end{array}$ & $\begin{array}{c}\text { Yes } \\
x^{2}(1, \mathrm{~N}=106)= \\
46.23^{*}\end{array}$ & $\begin{array}{c}\text { No } \\
x^{2}(1, \mathrm{~N}=88) \\
=5.50^{*}\end{array}$ & $\begin{array}{c}\text { Yes } \\
x^{2}(1, \mathrm{~N}=55) \\
=11.36^{*}\end{array}$ & 2 \\
\hline
\end{tabular}




\begin{tabular}{|c|c|c|c|c|c|c|}
\hline $\begin{array}{l}\text { Chart } \\
\text { Type }\end{array}$ & $\begin{array}{l}\text { Information } \\
\text { Category }\end{array}$ & Information Element & $\begin{array}{l}\text { Q1: Should it be } \\
\text { displayed to } \\
\text { execute } \\
\text { procedure? }\end{array}$ & $\begin{array}{l}\text { Q2: Displayed } \\
\text { at all times? }\end{array}$ & $\begin{array}{l}\text { Q3: Displayed } \\
\text { Initially? }\end{array}$ & $\begin{array}{l}\text { Importance } \\
\text { Level }\end{array}$ \\
\hline SID & Geography & $\begin{array}{l}\text { Parallels and Meridians } \\
\text { with AMAs, OROCAs, } \\
\text { MORAs }\end{array}$ & $\begin{array}{c}\text { Yes } \\
x^{2}(1, \mathrm{~N}=104)= \\
77.88^{*}\end{array}$ & $\begin{array}{c}\text { No } \\
x^{2}(1, \mathrm{~N}=97) \\
=26.81^{*}\end{array}$ & $\begin{array}{l}\text { No Preference } \\
x^{2}(1, N=74) \\
\quad=0.22\end{array}$ & $\begin{array}{l}\text { Not Yet } \\
\text { Determined }\end{array}$ \\
\hline SID & Geography & Parallels and Meridians & $\begin{array}{c}\text { Yes } \\
x^{2}(1, \mathrm{~N}=102)= \\
59.65^{*}\end{array}$ & $\begin{array}{c}\text { No } \\
x^{2}(1, \mathrm{~N}=90) \\
=45.51^{*}\end{array}$ & $\begin{array}{l}\text { No Preference } \\
x^{2}(1, \mathrm{~N}=77) \\
=1.05\end{array}$ & 3 \\
\hline SID & Geography & Water Features & $\begin{array}{c}\text { Yes } \\
x^{2}(1, \mathrm{~N}=110)= \\
80.33^{*}\end{array}$ & $\begin{array}{c}\text { No } \\
x^{2}(1, \mathrm{~N}= \\
102)=20.75^{*}\end{array}$ & $\begin{array}{l}\text { No Preference } \\
x^{2}(1, \mathrm{~N}=74) \\
=2.65\end{array}$ & $\begin{array}{l}\text { Not Yet } \\
\text { Determined }\end{array}$ \\
\hline SID & Geography & Cultural Features & $\begin{array}{c}\text { Yes } \\
x^{2}(1, \mathrm{~N}=106)= \\
51.66^{*}\end{array}$ & $\begin{array}{c}\text { No } \\
x^{2}(1, \mathrm{~N}=90) \\
=32.40^{*}\end{array}$ & $\begin{array}{l}\text { No Preference } \\
x^{2}(1, \mathrm{~N}=72) \\
\quad=0.50\end{array}$ & $\begin{array}{l}\text { Not Yet } \\
\text { Determined }\end{array}$ \\
\hline SID & Geography & Terrain Contours & $\begin{array}{c}\text { Yes } \\
x^{2}(1, \mathrm{~N}=110)= \\
98.33^{*}\end{array}$ & $\begin{array}{c}\text { No preference } \\
x^{2}(1, \mathrm{~N}= \\
107)=0.76\end{array}$ & & 1 \\
\hline SID & Geography & $\begin{array}{l}\text { Terrain Contour } \\
\text { Elevations }\end{array}$ & $\begin{array}{c}\text { Yes } \\
x^{2}(1, \mathrm{~N}=110)= \\
102.15^{*}\end{array}$ & $\begin{array}{c}\text { No preference } \\
x^{2}(1, N= \\
108)=0.33 \\
\end{array}$ & & 1 \\
\hline SID & Geography & Contour Interval Legend & $\begin{array}{c}\text { Yes } \\
x^{2}(1, \mathrm{~N}=108)= \\
78.37^{*}\end{array}$ & $\begin{array}{c}\text { No } \\
x^{2}(1, \mathrm{~N}= \\
100)=33.64^{*}\end{array}$ & $\begin{array}{l}\text { No Preference } \\
x^{2}(1, \mathrm{~N}=79) \\
=0.11\end{array}$ & $\begin{array}{l}\text { Not Yet } \\
\text { Determined }\end{array}$ \\
\hline SID & Geography & Spot Elevations & $\begin{array}{c}\text { Yes } \\
x^{2}(1, \mathrm{~N}=108)= \\
88.93^{*}\end{array}$ & $\begin{array}{c}\text { No preference } \\
x^{2}(1, N= \\
103)=1.17 \\
\end{array}$ & & 1 \\
\hline SID & Geography & $\begin{array}{l}\text { Highest Reference Point } \\
\text { (within neat lines) }\end{array}$ & $\begin{array}{c}\text { Yes } \\
x^{2}(1, \mathrm{~N}=110)= \\
106.04^{*}\end{array}$ & $\begin{array}{c}\text { Yes } \\
x^{2}(1, \mathrm{~N}= \\
109)=6.69^{*}\end{array}$ & & 1 \\
\hline SID & Geography & $\begin{array}{l}\text { International Boundaries } \\
\text { (higher criticality where } \\
\text { appropriate) }\end{array}$ & $\begin{array}{c}\text { Yes } \\
x^{2}(1, \mathrm{~N}=106)= \\
94.34^{*}\end{array}$ & $\begin{array}{c}\text { No } \\
x^{2}(1, \mathrm{~N}= \\
103)=19.66^{*}\end{array}$ & $\begin{array}{c}\text { Yes } \\
x^{2}(1, \mathrm{~N}=74) \\
=5.41^{*}\end{array}$ & 2 \\
\hline SID & Obstacle & $\begin{array}{l}\text { Obstacle Symbols and } \\
\text { Elevation }\end{array}$ & $\begin{array}{c}\text { Yes } \\
x^{2}(1, \mathrm{~N}=109)= \\
109.00^{*}\end{array}$ & $\begin{array}{c}\text { Yes } \\
x^{2}(1, \mathrm{~N}= \\
109)=8.82 * \\
\end{array}$ & & 1 \\
\hline SID & $\begin{array}{l}\text { Minimum } \\
\text { Area/Sector } \\
\text { Altitudes }\end{array}$ & $\begin{array}{l}\text { MSA Reference } \\
\text { Point/Center }\end{array}$ & $\begin{array}{c}\text { Yes } \\
x^{2}(1, \mathrm{~N}=109)= \\
101.15^{*}\end{array}$ & $\begin{array}{c}\text { No preference } \\
x^{2}(1, \mathrm{~N}= \\
107)=0.46\end{array}$ & & $\begin{array}{l}\text { Not Yet } \\
\text { Determined }\end{array}$ \\
\hline SID & $\begin{array}{l}\text { Minimum } \\
\text { Area/Sector } \\
\text { Altitudes }\end{array}$ & $\begin{array}{l}\text { MSA Distance when } \\
\text { other than } 25 \mathrm{~nm}\end{array}$ & $\begin{array}{c}\text { Yes } \\
x^{2}(1, \mathrm{~N}=109)= \\
101.15^{*}\end{array}$ & $\begin{array}{c}\text { No preference } \\
x^{2}(1, N= \\
107)=3.37 \\
\end{array}$ & & 2 \\
\hline SID & $\begin{array}{l}\text { Minimum } \\
\text { Area/Sector } \\
\text { Altitudes }\end{array}$ & MSA Sector Radials & $\begin{array}{c}\text { Yes } \\
x^{2}(1, \mathrm{~N}=109)= \\
109.00^{*}\end{array}$ & $\begin{array}{c}\text { No preference } \\
x^{2}(1, \mathrm{~N}= \\
109)=0.23\end{array}$ & & 1 \\
\hline
\end{tabular}




\begin{tabular}{|c|c|c|c|c|c|c|}
\hline $\begin{array}{l}\text { Chart } \\
\text { Type }\end{array}$ & $\begin{array}{l}\text { Information } \\
\text { Category }\end{array}$ & Information Element & $\begin{array}{l}\text { Q1: Should it be } \\
\text { displayed to } \\
\text { execute } \\
\text { procedure? }\end{array}$ & $\begin{array}{l}\text { Q2: Displayed } \\
\text { at all times? }\end{array}$ & $\begin{array}{l}\text { Q3: Displayed } \\
\text { Initially? }\end{array}$ & $\begin{array}{l}\text { Importance } \\
\text { Level }\end{array}$ \\
\hline SID & $\begin{array}{l}\text { Minimum } \\
\text { Area/Sector } \\
\text { Altitudes }\end{array}$ & $\begin{array}{l}\text { MSA Minimum } \\
\text { Altitudes }\end{array}$ & $\begin{array}{c}\text { Yes } \\
x^{2}(1, \mathrm{~N}=110)= \\
110.00^{*}\end{array}$ & $\begin{array}{c}\text { No preference } \\
x^{2}(1, N= \\
110)=3.64 \\
\end{array}$ & & 1 \\
\hline SID & $\begin{array}{l}\text { Minimum } \\
\text { Area/Sector } \\
\text { Altitudes }\end{array}$ & $\begin{array}{l}\text { Minimum Radar } \\
\text { Altitudes and Sectors }\end{array}$ & $\begin{array}{c}\text { Yes } \\
x^{2}(1, \mathrm{~N}=109)= \\
101.15^{*}\end{array}$ & $\begin{array}{c}\text { No preference } \\
x^{2}(1, \mathrm{~N}= \\
107)=0.46\end{array}$ & & 1 \\
\hline SID & $\begin{array}{l}\text { Minimum } \\
\text { Area/Sector } \\
\text { Altitudes }\end{array}$ & $\begin{array}{l}\text { AMA, OROCA, or grid } \\
\text { MORA where } \\
\text { established }\end{array}$ & $\begin{array}{c}\text { Yes } \\
x^{2}(1, \mathrm{~N}=103)= \\
83.97 *\end{array}$ & $\begin{array}{c}\text { No } \\
x^{2}(1, \mathrm{~N}=98) \\
=16.33^{*}\end{array}$ & $\begin{array}{c}\text { Yes } \\
x^{2}(1, \mathrm{~N}=69) \\
=6.39 *\end{array}$ & 2 \\
\hline SID & Navigation & FIR/UIR Boundaries & $\begin{array}{c}\text { Yes } \\
x^{2}(1, \mathrm{~N}=94)= \\
64.72 *\end{array}$ & $\begin{array}{c}\text { No } \\
x^{2}(1, \mathrm{~N}=86) \\
=22.51^{*}\end{array}$ & $\begin{array}{c}\text { Yes } \\
x^{2}(1, \mathrm{~N}=65) \\
=5.55^{*}\end{array}$ & 2 \\
\hline SID & Navigation & $\begin{array}{l}\text { Prohibited, Restricted } \\
\text { and Danger Airspace } \\
\text { Graphic }\end{array}$ & $\begin{array}{c}\text { Yes } \\
x^{2}(1, \mathrm{~N}=110)= \\
110.00^{*}\end{array}$ & $\begin{array}{c}\text { Yes } \\
x^{2}(1, \mathrm{~N}= \\
110)=10.51^{*}\end{array}$ & & 1 \\
\hline SID & Navigation & $\begin{array}{l}\text { Prohibited, Restricted } \\
\text { and Danger Airspace } \\
\text { Label }\end{array}$ & $\begin{array}{c}\text { Yes } \\
x^{2}(1, \mathrm{~N}=110)= \\
110.00^{*}\end{array}$ & $\begin{array}{c}\text { No preference } \\
x^{2}(1, \mathrm{~N}= \\
110)=0.58\end{array}$ & & 1 \\
\hline SID & Navigation & $\begin{array}{l}\text { Prohibited, Restricted } \\
\text { and Danger Airspace } \\
\text { Narrative }\end{array}$ & $\begin{array}{c}\text { Yes } \\
x^{2}(1, \mathrm{~N}=109)= \\
105.04^{*}\end{array}$ & $\begin{array}{c}\text { No } \\
x^{2}(1, \mathrm{~N}= \\
108)=31.15^{*}\end{array}$ & $\begin{array}{c}\text { Yes } \\
x^{2}(1, \mathrm{~N}=83) \\
=5.31^{*}\end{array}$ & 2 \\
\hline SID & Navigation & $\begin{array}{l}\text { Special Use Airspace - } \\
\text { Other }\end{array}$ & $\begin{array}{c}\text { Yes } \\
x^{2}(1, \mathrm{~N}=110)= \\
102.15^{*}\end{array}$ & $\begin{array}{c}\text { No } \\
x^{2}(1, \mathrm{~N}= \\
108)=13.37 *\end{array}$ & $\begin{array}{c}\text { Yes } \\
x^{2}(1, \mathrm{~N}=73) \\
=14.92^{*}\end{array}$ & $\begin{array}{l}\text { Not Yet } \\
\text { Determined }\end{array}$ \\
\hline SID & Navigation & Transition Altitude & $\begin{array}{c}\text { Yes } \\
x^{2}(1, \mathrm{~N}=108)= \\
104.04^{*}\end{array}$ & $\begin{array}{c}\text { No preference } \\
x^{2}(1, N= \\
107)=0.08 \\
\end{array}$ & & 1 \\
\hline SID & $\begin{array}{l}\text { Airport } \\
\text { Information }\end{array}$ & Runway Layout & $\begin{array}{c}\text { Yes } \\
x^{2}(1, \mathrm{~N}=108)= \\
100.15^{*}\end{array}$ & $\begin{array}{c}\text { Yes } \\
x^{2}(1, \mathrm{~N}= \\
106)=29.58^{*}\end{array}$ & & 1 \\
\hline SID & $\begin{array}{l}\text { Airport } \\
\text { Information }\end{array}$ & Airport Elevation & $\begin{array}{c}\text { Yes } \\
x^{2}(1, \mathrm{~N}=110)= \\
98.33^{*}\end{array}$ & $\begin{array}{c}\text { Yes } \\
x^{2}(1, \mathrm{~N}= \\
107)=8.98^{*}\end{array}$ & & 1 \\
\hline SID & $\begin{array}{l}\text { Airport } \\
\text { Information }\end{array}$ & Other Airport Symbols & $\begin{array}{c}\text { Yes } \\
x^{2}(1, \mathrm{~N}=109)= \\
93.59 *\end{array}$ & $\begin{array}{c}\text { No } \\
x^{2}(1, \mathrm{~N}= \\
105)=22.87^{*}\end{array}$ & $\begin{array}{c}\text { Yes } \\
x^{2}(1, \mathrm{~N}=77) \\
=19.75^{*}\end{array}$ & 2 \\
\hline SID & $\begin{array}{l}\text { Airport } \\
\text { Information }\end{array}$ & Other Airport Names & $\begin{array}{c}\text { Yes } \\
x^{2}(1, \mathrm{~N}=109)= \\
89.92^{*}\end{array}$ & $\begin{array}{c}\text { No } \\
x^{2}(1, \mathrm{~N}= \\
104)=30.15^{*}\end{array}$ & $\begin{array}{c}\text { No Preference } \\
x^{2}(1, \mathrm{~N}=80) \\
=3.20\end{array}$ & $\begin{array}{l}\text { Not Yet } \\
\text { Determined }\end{array}$ \\
\hline SID & $\begin{array}{l}\text { Airport } \\
\text { Information }\end{array}$ & Other Airport Elevations & $\begin{array}{c}\text { Yes } \\
x^{2}(1, \mathrm{~N}=109)= \\
75.97^{*}\end{array}$ & $\begin{array}{c}\text { No } \\
x^{2}(1, \mathrm{~N}= \\
100)=49.00^{*}\end{array}$ & $\begin{array}{l}\text { No Preference } \\
x^{2}(1, \mathrm{~N}=85) \\
\quad=1.99\end{array}$ & $\begin{array}{l}\text { Not Yet } \\
\text { Determined }\end{array}$ \\
\hline
\end{tabular}




\begin{tabular}{|c|c|c|c|c|c|c|}
\hline $\begin{array}{l}\text { Chart } \\
\text { Type }\end{array}$ & $\begin{array}{l}\text { Information } \\
\text { Category }\end{array}$ & Information Element & $\begin{array}{l}\text { Q1: Should it be } \\
\text { displayed to } \\
\text { execute } \\
\text { procedure? }\end{array}$ & $\begin{array}{l}\text { Q2: Displayed } \\
\text { at all times? }\end{array}$ & $\begin{array}{l}\text { Q3: Displayed } \\
\text { Initially? }\end{array}$ & $\begin{array}{l}\text { Importance } \\
\text { Level }\end{array}$ \\
\hline SID & $\begin{array}{l}\text { Airport } \\
\text { Information }\end{array}$ & $\begin{array}{l}\text { Distances from airport to } \\
\text { first fix on SID }\end{array}$ & $\begin{array}{c}\text { Yes } \\
x^{2}(1, \mathrm{~N}=110)= \\
102.15^{*}\end{array}$ & $\begin{array}{c}\text { No preference } \\
x^{2}(1, \mathrm{~N}= \\
108)=3.00\end{array}$ & & 1 \\
\hline SID & $\begin{array}{l}\text { Navaid Used to } \\
\text { Form Leg of } \\
\text { Procedure }\end{array}$ & Navaid Symbol & $\begin{array}{c}\text { Yes } \\
x^{2}(1, \mathrm{~N}=110)= \\
106.04^{*}\end{array}$ & $\begin{array}{c}\text { Yes } \\
x^{2}(1, \mathrm{~N}= \\
109)=41.18^{*}\end{array}$ & & 1 \\
\hline SID & $\begin{array}{l}\text { Navaid Used to } \\
\text { Form Leg of } \\
\text { Procedure }\end{array}$ & Navaid Name & $\begin{array}{c}\text { Yes } \\
x^{2}(1, \mathrm{~N}=110)= \\
110.00^{*}\end{array}$ & $\begin{array}{c}\text { Yes } \\
x^{2}(1, \mathrm{~N}= \\
110)=14.55^{*}\end{array}$ & & 1 \\
\hline SID & $\begin{array}{l}\text { Navaid Used to } \\
\text { Form Leg of } \\
\text { Procedure }\end{array}$ & Navaid Identifier & $\begin{array}{c}\text { Yes } \\
x^{2}(1, \mathrm{~N}=110)= \\
110.00^{*}\end{array}$ & $\begin{array}{c}\text { Yes } \\
x^{2}(1, \mathrm{~N}= \\
110)=34.95^{*}\end{array}$ & & 1 \\
\hline SID & $\begin{array}{l}\text { Navaid Used to } \\
\text { Form Leg of } \\
\text { Procedure }\end{array}$ & $\begin{array}{l}\text { Navaid } \\
\text { Frequency/Channel }\end{array}$ & $\begin{array}{c}\text { Yes } \\
x^{2}(1, \mathrm{~N}=110)= \\
110.00^{*}\end{array}$ & $\begin{array}{c}\text { Yes } \\
x^{2}(1, \mathrm{~N}= \\
110)=16.04^{*}\end{array}$ & & 1 \\
\hline SID & $\begin{array}{l}\text { Navaid Used to } \\
\text { Form Leg of } \\
\text { Procedure }\end{array}$ & Navaid Morse Code & $\begin{array}{c}\text { Yes } \\
x^{2}(1, \mathrm{~N}=110)= \\
76.95^{*}\end{array}$ & $\begin{array}{c}\text { No } \\
x^{2}(1, \mathrm{~N}= \\
101)=55.69 *\end{array}$ & $\begin{array}{c}\text { No Preference } \\
x^{2}(1, N=88) \\
\quad=0.73\end{array}$ & $\begin{array}{l}\text { Not Yet } \\
\text { Determined }\end{array}$ \\
\hline SID & $\begin{array}{l}\text { Navaid Used to } \\
\text { Form Leg of } \\
\text { Procedure }\end{array}$ & $\begin{array}{l}\text { DME Availability (Text } \\
\text { information) }\end{array}$ & $\begin{array}{c}\text { Yes } \\
x^{2}(1, \mathrm{~N}=110)= \\
80.33^{*}\end{array}$ & $\begin{array}{c}\text { No } \\
x^{2}(1, \mathrm{~N}= \\
102)=20.75^{*}\end{array}$ & $\begin{array}{c}\text { No Preference } \\
x^{2}(1, \mathrm{~N}=74) \\
=1.95\end{array}$ & 2 \\
\hline SID & $\begin{array}{l}\text { Navaid Used to } \\
\text { Form Leg of } \\
\text { Procedure }\end{array}$ & Navaid Class & $\begin{array}{c}\text { Yes } \\
x^{2}(1, \mathrm{~N}=109)= \\
54.39^{*}\end{array}$ & $\begin{array}{c}\text { No } \\
x^{2}(1, \mathrm{~N}=93) \\
=60.48^{*}\end{array}$ & $\begin{array}{l}\text { No Preference } \\
x^{2}(1, \mathrm{~N}=84) \\
=1.71\end{array}$ & 3 \\
\hline SID & $\begin{array}{l}\text { Navaid Used to } \\
\text { Form Leg of } \\
\text { Procedure }\end{array}$ & $\begin{array}{l}\text { Navaid } \\
\text { Latitude/Longitude }\end{array}$ & $\begin{array}{c}\text { Yes } \\
x^{2}(1, \mathrm{~N}=110)= \\
37.24^{*}\end{array}$ & $\begin{array}{c}\text { No } \\
x^{2}(1, \mathrm{~N}=87) \\
=57.94^{*}\end{array}$ & $\begin{array}{c}\text { No } \\
x^{2}(1, \mathrm{~N}=79) \\
=10.65^{*}\end{array}$ & 3 \\
\hline SID & $\begin{array}{l}\text { Navaid Used to } \\
\text { Form Fixes }\end{array}$ & Navaid Symbol & $\begin{array}{c}\text { Yes } \\
x^{2}(1, \mathrm{~N}=110)= \\
110.00^{*}\end{array}$ & $\begin{array}{c}\text { Yes } \\
x^{2}(1, \mathrm{~N}= \\
110)=34.95^{*}\end{array}$ & & 1 \\
\hline SID & $\begin{array}{l}\text { Navaid Used to } \\
\text { Form Fixes }\end{array}$ & Navaid Name & $\begin{array}{c}\text { Yes } \\
x^{2}(1, \mathrm{~N}=110)= \\
110.00^{*}\end{array}$ & $\begin{array}{c}\text { Yes } \\
x^{2}(1, \mathrm{~N}= \\
110)=10.51^{*}\end{array}$ & & 1 \\
\hline SID & $\begin{array}{l}\text { Navaid Used to } \\
\text { Form Fixes }\end{array}$ & Navaid Identifier & $\begin{array}{c}\text { Yes } \\
x^{2}(1, \mathrm{~N}=110)= \\
110.00^{*}\end{array}$ & $\begin{array}{c}\text { Yes } \\
x^{2}(1, \mathrm{~N}= \\
110)=30.58^{*}\end{array}$ & & 1 \\
\hline SID & $\begin{array}{l}\text { Navaid Used to } \\
\text { Form Fixes }\end{array}$ & $\begin{array}{l}\text { Navaid } \\
\text { Frequency/Channel }\end{array}$ & $\begin{array}{c}\text { Yes } \\
x^{2}(1, \mathrm{~N}=109)= \\
105.04^{*}\end{array}$ & $\begin{array}{c}\text { Yes } \\
x^{2}(1, \mathrm{~N}= \\
108)=23.15^{*}\end{array}$ & & 1 \\
\hline SID & $\begin{array}{l}\text { Navaid Used to } \\
\text { Form Fixes }\end{array}$ & Navaid Morse Code & $\begin{array}{c}\text { Yes } \\
x^{2}(1, \mathrm{~N}=110)= \\
70.40^{*}\end{array}$ & $\begin{array}{c}\text { No } \\
x^{2}(1, \mathrm{~N}=99) \\
=53.83^{*}\end{array}$ & $\begin{array}{l}\text { No Preference } \\
x^{2}(1, \mathrm{~N}=86) \\
=0.05\end{array}$ & $\begin{array}{l}\text { Not Yet } \\
\text { Determined }\end{array}$ \\
\hline SID & $\begin{array}{l}\text { Navaid Used to } \\
\text { Form Fixes }\end{array}$ & $\begin{array}{l}\text { DME Availability (Text } \\
\text { information) }\end{array}$ & $\begin{array}{c}\text { Yes } \\
x^{2}(1, \mathrm{~N}=110)= \\
76.95^{*}\end{array}$ & $\begin{array}{c}\text { No } \\
x^{2}(1, \mathrm{~N}= \\
101)=27.81^{*}\end{array}$ & $\begin{array}{l}\text { No Preference } \\
\begin{array}{c}x^{2}(1, N=77) \\
=1.57\end{array}\end{array}$ & 2 \\
\hline
\end{tabular}




\begin{tabular}{|c|c|c|c|c|c|c|}
\hline $\begin{array}{l}\text { Chart } \\
\text { Type }\end{array}$ & $\begin{array}{l}\text { Information } \\
\text { Category }\end{array}$ & Information Element & $\begin{array}{l}\text { Q1: Should it be } \\
\text { displayed to } \\
\text { execute } \\
\text { procedure? }\end{array}$ & $\begin{array}{l}\text { Q2: Displayed } \\
\text { at all times? }\end{array}$ & $\begin{array}{l}\text { Q3: Displayed } \\
\text { Initially? }\end{array}$ & $\begin{array}{l}\text { Importance } \\
\text { Level }\end{array}$ \\
\hline SID & $\begin{array}{l}\text { Navaid Used to } \\
\text { Form Fixes }\end{array}$ & Navaid Class & $\begin{array}{c}\text { Yes } \\
x^{2}(1, \mathrm{~N}=109)= \\
48.89^{*}\end{array}$ & $\begin{array}{c}\text { No } \\
x^{2}(1, \mathrm{~N}=91) \\
=61.81^{*}\end{array}$ & $\begin{array}{l}\text { No Preference } \\
x^{2}(1, \mathrm{~N}=83) \\
=1.46\end{array}$ & 3 \\
\hline SID & $\begin{array}{l}\text { Navaid Used to } \\
\text { Form Fixes }\end{array}$ & $\begin{array}{l}\text { Navaid } \\
\text { Latitude/Longitude }\end{array}$ & $\begin{array}{c}\text { Yes } \\
x^{2}(1, \mathrm{~N}=109)= \\
41.18^{*}\end{array}$ & $\begin{array}{c}\text { No } \\
x^{2}(1, \mathrm{~N}=88) \\
=65.64^{*}\end{array}$ & $\begin{array}{c}\text { No } \\
x^{2}(1, \mathrm{~N}=82) \\
=4.88^{*}\end{array}$ & 3 \\
\hline SID & $\begin{array}{l}\text { Navaid Used to } \\
\text { Form Fixes }\end{array}$ & $\begin{array}{l}\text { Navaid Radials/Bearings } \\
\text { that form fixes }\end{array}$ & $\begin{array}{c}\text { Yes } \\
x^{2}(1, N=110)= \\
110.00^{*}\end{array}$ & $\begin{array}{c}\text { No preference } \\
x^{2}(1, N= \\
110)=0.91 \\
\end{array}$ & & 1 \\
\hline SID & $\begin{array}{l}\text { Navaid Used to } \\
\text { Form Fixes }\end{array}$ & $\begin{array}{l}\text { DME Distances that } \\
\text { form fixes }\end{array}$ & $\begin{array}{c}\text { Yes } \\
x^{2}(1, N=109)= \\
105.04^{*}\end{array}$ & $\begin{array}{c}\text { Yes } \\
x^{2}(1, \mathrm{~N}= \\
108)=6.26^{*}\end{array}$ & & 1 \\
\hline SID & $\begin{array}{l}\text { Instrument } \\
\text { Procedure } \\
\text { Courses/Tracks }\end{array}$ & $\begin{array}{l}\text { Symbol (e.g., line style, } \\
\text { etc.) }\end{array}$ & $\begin{array}{c}\text { Yes } \\
x^{2}(1, \mathrm{~N}=108)= \\
108.00^{*}\end{array}$ & $\begin{array}{c}\text { Yes } \\
x^{2}(1, \mathrm{~N}= \\
108)=37.93^{*}\end{array}$ & & 1 \\
\hline SID & $\begin{array}{l}\text { Instrument } \\
\text { Procedure } \\
\text { Courses/Tracks }\end{array}$ & $\begin{array}{l}\text { Identifier (i.e., } \\
\text { CNOG8.AVE) }\end{array}$ & $\begin{array}{c}\text { Yes } \\
x^{2}(1, \mathrm{~N}=109)= \\
105.04^{*}\end{array}$ & $\begin{array}{c}\text { Yes } \\
x^{2}(1, \mathrm{~N}= \\
108)=23.15^{*}\end{array}$ & & 1 \\
\hline SID & $\begin{array}{l}\text { Course } \\
\text { Definition }\end{array}$ & Heading & $\begin{array}{c}\text { Yes } \\
x^{2}(1, \mathrm{~N}=109)= \\
109.00^{*}\end{array}$ & $\begin{array}{c}\text { Yes } \\
x^{2}(1, \mathrm{~N}= \\
109)=63.20^{*}\end{array}$ & & 1 \\
\hline SID & $\begin{array}{l}\text { Course } \\
\text { Definition }\end{array}$ & Track & $\begin{array}{c}\text { Yes } \\
x^{2}(1, \mathrm{~N}=109)= \\
109.00^{*}\end{array}$ & $\begin{array}{c}\text { Yes } \\
x^{2}(1, \mathrm{~N}= \\
109)=63.20^{*}\end{array}$ & & 1 \\
\hline SID & $\begin{array}{l}\text { Course } \\
\text { Definition }\end{array}$ & Radial & $\begin{array}{c}\text { Yes } \\
x^{2}(1, \mathrm{~N}=110)= \\
110.00^{*}\end{array}$ & $\begin{array}{c}\text { Yes } \\
x^{2}(1, \mathrm{~N}= \\
110)=58.18^{*}\end{array}$ & & 1 \\
\hline SID & $\begin{array}{l}\text { Course } \\
\text { Definition }\end{array}$ & Segment Mileages & $\begin{array}{c}\text { Yes } \\
x^{2}(1, \mathrm{~N}=110)= \\
110.00^{*}\end{array}$ & $\begin{array}{c}\text { Yes } \\
x^{2}(1, \mathrm{~N}= \\
110)=20.95^{*}\end{array}$ & & 1 \\
\hline SID & $\begin{array}{l}\text { Course } \\
\text { Definition }\end{array}$ & MEA/MOCA & $\begin{array}{c}\text { Yes } \\
x^{2}(1, \mathrm{~N}=110)= \\
102.15^{*}\end{array}$ & $\begin{array}{c}\text { No preference } \\
x^{2}(1, \mathrm{~N}= \\
108)=0.04\end{array}$ & & 1 \\
\hline SID & $\begin{array}{l}\text { Course } \\
\text { Definition }\end{array}$ & $\begin{array}{l}\text { VOR Change Over } \\
\text { Points }\end{array}$ & $\begin{array}{c}\text { Yes } \\
x^{2}(1, N=110)= \\
87.31^{*}\end{array}$ & $\begin{array}{c}\text { No preference } \\
x^{2}(1, N= \\
104)=3.12 \\
\end{array}$ & & 2 \\
\hline SID & Holding Pattern & $\begin{array}{l}\text { Holding Pattern } \\
\text { Depiction }\end{array}$ & $\begin{array}{c}\text { Yes } \\
x^{2}(1, \mathrm{~N}=110)= \\
110.00^{*}\end{array}$ & $\begin{array}{c}\text { Yes } \\
x^{2}(1, \mathrm{~N}= \\
110)=20.95^{*}\end{array}$ & & 1 \\
\hline SID & Holding Pattern & Holding Pattern Courses & $\begin{array}{c}\text { Yes } \\
x^{2}(1, \mathrm{~N}=110)= \\
110.00^{*}\end{array}$ & $\begin{array}{c}\text { Yes } \\
x^{2}(1, \mathrm{~N}= \\
110)=5.24^{*}\end{array}$ & & 1 \\
\hline SID & Holding Pattern & $\begin{array}{l}\text { Holding Pattern Leg } \\
\text { Length }\end{array}$ & $\begin{array}{c}\text { Yes } \\
x^{2}(1, \mathrm{~N}=110)= \\
110.00^{*}\end{array}$ & $\begin{array}{c}\text { No preference } \\
x^{2}(1, N= \\
110)=0.04\end{array}$ & & 1 \\
\hline
\end{tabular}




\begin{tabular}{|c|c|c|c|c|c|c|}
\hline $\begin{array}{l}\text { Chart } \\
\text { Type }\end{array}$ & $\begin{array}{l}\text { Information } \\
\text { Category }\end{array}$ & Information Element & $\begin{array}{l}\text { Q1: Should it be } \\
\text { displayed to } \\
\text { execute } \\
\text { procedure? }\end{array}$ & $\begin{array}{l}\text { Q2: Displayed } \\
\text { at all times? }\end{array}$ & $\begin{array}{l}\text { Q3: Displayed } \\
\text { Initially? }\end{array}$ & $\begin{array}{l}\text { Importance } \\
\text { Level }\end{array}$ \\
\hline SID & Holding Pattern & Holding Pattern Time & $\begin{array}{c}\text { Yes } \\
x^{2}(1, \mathrm{~N}=110)= \\
102.15^{*}\end{array}$ & $\begin{array}{c}\text { No } \\
x^{2}(1, \mathrm{~N}= \\
108)=19.59 *\end{array}$ & $\begin{array}{c}\text { Yes } \\
x^{2}(1, \mathrm{~N}=77) \\
=12.48^{*}\end{array}$ & 2 \\
\hline SID & Holding Pattern & Holding Pattern Speed & $\begin{array}{c}\text { Yes } \\
x^{2}(1, \mathrm{~N}=110)= \\
98.33^{*}\end{array}$ & $\begin{array}{c}\text { No } \\
x^{2}(1, \mathrm{~N}= \\
107)=24.31^{*}\end{array}$ & $\begin{array}{c}\text { Yes } \\
x^{2}(1, \mathrm{~N}=79) \\
=13.78^{*}\end{array}$ & 2 \\
\hline SID & Holding Pattern & Holding Pattern Altitude & $\begin{array}{c}\text { Yes } \\
x^{2}(1, \mathrm{~N}=110)= \\
102.15^{*}\end{array}$ & $\begin{array}{c}\text { No preference } \\
x^{2}(1, N= \\
108)=1.33 \\
\end{array}$ & & 1 \\
\hline SID & Transitions & $\begin{array}{l}\text { Transition Courses } \\
\text { depiction }\end{array}$ & $\begin{array}{c}\text { Yes } \\
x^{2}(1, \mathrm{~N}=109)= \\
109.00^{*}\end{array}$ & $\begin{array}{c}\text { Yes } \\
x^{2}(1, \mathrm{~N}= \\
109)=23.86^{*}\end{array}$ & & 1 \\
\hline SID & Transitions & Transition Name & $\begin{array}{c}\text { Yes } \\
x^{2}(1, \mathrm{~N}=108)= \\
108.00^{*}\end{array}$ & $\begin{array}{c}\text { Yes } \\
x^{2}(1, \mathrm{~N}= \\
108)=12.00^{*}\end{array}$ & & 1 \\
\hline SID & Transitions & $\begin{array}{l}\text { Transition Courses } \\
\text { computer codes }\end{array}$ & $\begin{array}{c}\text { Yes } \\
x^{2}(1, \mathrm{~N}=104)= \\
84.96^{*}\end{array}$ & $\begin{array}{c}\text { No } \\
x^{2}(1, \mathrm{~N}=99) \\
=26.27^{*}\end{array}$ & $\begin{array}{c}\text { Yes } \\
x^{2}(1, \mathrm{~N}=75) \\
=7.05^{*}\end{array}$ & 2 \\
\hline SID & Transitions & $\begin{array}{l}\text { Transition Course - } \\
\text { Magnetic Values }\end{array}$ & $\begin{array}{c}\text { Yes } \\
x^{2}(1, \mathrm{~N}=107)= \\
103.04^{*}\end{array}$ & $\begin{array}{c}\text { No preference } \\
x^{2}(1, \mathrm{~N}= \\
106)=0.15\end{array}$ & & $\begin{array}{l}\text { Not Yet } \\
\text { Determined }\end{array}$ \\
\hline SID & Transitions & $\begin{array}{l}\text { Transition Courses - } \\
\text { MEAs, MOCAs }\end{array}$ & $\begin{array}{c}\text { Yes } \\
x^{2}(1, \mathrm{~N}=108)= \\
96.33^{*}\end{array}$ & $\begin{array}{c}\text { No preference } \\
x^{2}(1, N= \\
105)=2.75\end{array}$ & & $\begin{array}{l}\text { Not Yet } \\
\text { Determined }\end{array}$ \\
\hline SID & Transitions & $\begin{array}{l}\text { Transition Courses - } \\
\text { segment milages }\end{array}$ & $\begin{array}{c}\text { Yes } \\
x^{2}(1, \mathrm{~N}=108)= \\
104.04^{*}\end{array}$ & $\begin{array}{c}\text { No preference } \\
x^{2}(1, N= \\
107)=0.46 \\
\end{array}$ & & $\begin{array}{l}\text { Not Yet } \\
\text { Determined }\end{array}$ \\
\hline SID & Transitions & $\begin{array}{l}\text { Transition Course notes } \\
\text { (e.g., DME required) }\end{array}$ & $\begin{array}{c}\text { Yes } \\
x^{2}(1, \mathrm{~N}=107)= \\
99.15^{*}\end{array}$ & $\begin{array}{c}\text { No } \\
x^{2}(1, \mathrm{~N}= \\
105)=24.77^{*}\end{array}$ & $\begin{array}{c}\text { Yes } \\
x^{2}(1, \mathrm{~N}=78) \\
=14.82^{*}\end{array}$ & 2 \\
\hline SID & Transitions & Transition Text & $\begin{array}{c}\text { Yes } \\
x^{2}(1, \mathrm{~N}=108)= \\
85.33^{*}\end{array}$ & $\begin{array}{c}\text { No } \\
x^{2}(1, \mathrm{~N}= \\
102)=48.04 *\end{array}$ & $\begin{array}{c}\text { Yes } \\
x^{2}(1, \mathrm{~N}=86) \\
=22.51^{*}\end{array}$ & 2 \\
\hline SID & $\begin{array}{l}\text { Intersection/ } \\
\text { Fixes on } \\
\text { Procedure }\end{array}$ & Symbol & $\begin{array}{c}\text { Yes } \\
x^{2}(1, \mathrm{~N}=110)= \\
110.00^{*}\end{array}$ & $\begin{array}{c}\text { Yes } \\
x^{2}(1, \mathrm{~N}= \\
110)=58.18^{*}\end{array}$ & & 1 \\
\hline SID & $\begin{array}{l}\text { Intersection/ } \\
\text { Fixes on } \\
\text { Procedure } \\
\end{array}$ & Names & $\begin{array}{c}\text { Yes } \\
x^{2}(1, \mathrm{~N}=109)= \\
109.00^{*}\end{array}$ & $\begin{array}{c}\text { Yes } \\
x^{2}(1, \mathrm{~N}= \\
109)=25.77 *\end{array}$ & & 1 \\
\hline SID & $\begin{array}{l}\text { Intersection/ } \\
\text { Fixes on } \\
\text { Procedure }\end{array}$ & Identifier & $\begin{array}{c}\text { Yes } \\
x^{2}(1, \mathrm{~N}=109)= \\
109.00^{*}\end{array}$ & $\begin{array}{c}\text { Yes } \\
x^{2}(1, \mathrm{~N}= \\
109)=29.81^{*}\end{array}$ & & 1 \\
\hline SID & $\begin{array}{l}\text { Intersection/ } \\
\text { Fixes on } \\
\text { Procedure }\end{array}$ & Latitude/Longitudes & $\begin{array}{c}\text { Yes } \\
x^{2}(1, \mathrm{~N}=110)= \\
47.13^{*}\end{array}$ & $\begin{array}{c}\text { No } \\
x^{2}(1, \mathrm{~N}=91) \\
=49.33^{*}\end{array}$ & $\begin{array}{c}\text { No } \\
x^{2}(1, \mathrm{~N}=79) \\
=6.70^{*}\end{array}$ & 3 \\
\hline
\end{tabular}




\begin{tabular}{|c|c|c|c|c|c|c|}
\hline $\begin{array}{l}\text { Chart } \\
\text { Type }\end{array}$ & $\begin{array}{l}\text { Information } \\
\text { Category }\end{array}$ & Information Element & $\begin{array}{l}\text { Q1: Should it be } \\
\text { displayed to } \\
\text { execute } \\
\text { procedure? }\end{array}$ & $\begin{array}{l}\text { Q2: Displayed } \\
\text { at all times? }\end{array}$ & $\begin{array}{l}\text { Q3: Displayed } \\
\text { Initially? }\end{array}$ & $\begin{array}{l}\text { Importance } \\
\text { Level }\end{array}$ \\
\hline SID & $\begin{array}{l}\text { Intersection/ } \\
\text { Fixes on } \\
\text { Procedure }\end{array}$ & MRA & $\begin{array}{c}\text { Yes } \\
x^{2}(1, \mathrm{~N}=109)= \\
79.35^{*}\end{array}$ & $\begin{array}{c}\text { No } \\
x^{2}(1, \mathrm{~N}= \\
101)=21.87^{*}\end{array}$ & $\begin{array}{l}\text { No Preference } \\
x^{2}(1, \mathrm{~N}=74) \\
=1.95\end{array}$ & 2 \\
\hline SID & $\begin{array}{l}\text { Textual } \\
\text { Information }\end{array}$ & Runway departure text & $\begin{array}{c}\text { Yes } \\
x^{2}(1, \mathrm{~N}=109)= \\
109.00^{*}\end{array}$ & $\begin{array}{c}\text { No preference } \\
x^{2}(1, N= \\
109)=2.65\end{array}$ & & $\begin{array}{l}\text { Not Yet } \\
\text { Determined }\end{array}$ \\
\hline SID & $\begin{array}{l}\text { Textual } \\
\text { Information }\end{array}$ & Climb Gradient - ATC & $\begin{array}{c}\text { Yes } \\
x^{2}(1, N=110)= \\
106.04^{*}\end{array}$ & $\begin{array}{c}\text { No } \\
x^{2}(1, \mathrm{~N}= \\
109)=4.05^{*}\end{array}$ & $\begin{array}{c}\text { Yes } \\
x^{2}(1, \mathrm{~N}=65) \\
=31.15^{*}\end{array}$ & 2 \\
\hline SID & $\begin{array}{l}\text { Textual } \\
\text { Information }\end{array}$ & $\begin{array}{l}\text { Climb Gradient - } \\
\text { Obstacle }\end{array}$ & $\begin{array}{c}\text { Yes } \\
x^{2}(1, \mathrm{~N}=110)= \\
106.04^{*}\end{array}$ & $\begin{array}{c}\text { No preference } \\
x^{2}(1, N= \\
109)=0.08\end{array}$ & & $\begin{array}{l}\text { Not Yet } \\
\text { Determined }\end{array}$ \\
\hline SID & $\begin{array}{l}\text { Textual } \\
\text { Information }\end{array}$ & Transition Text & $\begin{array}{c}\text { Yes } \\
x^{2}(1, \mathrm{~N}=109)= \\
101.15^{*}\end{array}$ & $\begin{array}{c}\text { No } \\
x^{2}(1, \mathrm{~N}= \\
107)=17.28^{*}\end{array}$ & $\begin{array}{c}\text { Yes } \\
x^{2}(1, \mathrm{~N}=75) \\
=32.01^{*}\end{array}$ & 2 \\
\hline SID & $\begin{array}{l}\text { Textual } \\
\text { Information }\end{array}$ & Notes & $\begin{array}{c}\text { Yes } \\
x^{2}(1, N=106)= \\
94.34^{*}\end{array}$ & $\begin{array}{c}\text { No } \\
x^{2}(1, \mathrm{~N}= \\
103)=73.49 *\end{array}$ & $\begin{array}{c}\text { Yes } \\
x^{2}(1, \mathrm{~N}=95) \\
=29.57^{*}\end{array}$ & 2 \\
\hline SID & $\begin{array}{l}\text { Textual } \\
\text { Information }\end{array}$ & Noise Abatement & $\begin{array}{c}\text { Yes } \\
x^{2}(1, \mathrm{~N}=110)= \\
94.58^{*}\end{array}$ & $\begin{array}{c}\text { No } \\
x^{2}(1, \mathrm{~N}= \\
106)=54.49 *\end{array}$ & $\begin{array}{c}\text { Yes } \\
x^{2}(1, \mathrm{~N}=91) \\
=26.38^{*}\end{array}$ & 2 \\
\hline SID & $\begin{array}{l}\text { Textual } \\
\text { Information }\end{array}$ & $\begin{array}{l}\text { Performance limitations } \\
\text { (e.g., bank limits) }\end{array}$ & $\begin{array}{c}\text { Yes } \\
x^{2}(1, \mathrm{~N}=110)= \\
98.33^{*}\end{array}$ & $\begin{array}{c}\text { No } \\
x^{2}(1, \mathrm{~N}= \\
107)=7.86^{*}\end{array}$ & $\begin{array}{c}\text { Yes } \\
x^{2}(1, \mathrm{~N}=68) \\
=11.53^{*}\end{array}$ & 2 \\
\hline SID & $\begin{array}{l}\text { Textual } \\
\text { Information }\end{array}$ & Text-Only Procedures & $\begin{array}{c}\text { Yes } \\
x^{2}(1, \mathrm{~N}=110)= \\
102.15^{*}\end{array}$ & $\begin{array}{c}\text { No } \\
x^{2}(1, \mathrm{~N}= \\
108)=23.15^{*}\end{array}$ & $\begin{array}{c}\text { Yes } \\
x^{2}(1, \mathrm{~N}=79) \\
=15.51^{*}\end{array}$ & 2 \\
\hline SID & $\begin{array}{l}\text { Textual } \\
\text { Information }\end{array}$ & General Notes & $\begin{array}{c}\text { Yes } \\
x^{2}(1, \mathrm{~N}=110)= \\
94.58^{*}\end{array}$ & $\begin{array}{c}\text { No } \\
x^{2}(1, \mathrm{~N}= \\
106)=73.06^{*}\end{array}$ & $\begin{array}{c}\text { Yes } \\
x^{2}(1, \mathrm{~N}=97) \\
=5.45^{*}\end{array}$ & 2 \\
\hline SID & $\begin{array}{l}\text { Textual } \\
\text { Information }\end{array}$ & Procedural Data Notes & $\begin{array}{c}\text { Yes } \\
x^{2}(1, \mathrm{~N}=109)= \\
101.15^{*}\end{array}$ & $\begin{array}{c}\text { No } \\
x^{2}(1, \mathrm{~N}= \\
107)=55.41^{*}\end{array}$ & $\begin{array}{c}\text { Yes } \\
x^{2}(1, \mathrm{~N}=92) \\
=7.35^{*}\end{array}$ & 2 \\
\hline SID & $\begin{array}{l}\text { Textual } \\
\text { Information }\end{array}$ & $\begin{array}{l}\text { Crossing Altitude } \\
\text { Restrictions }\end{array}$ & $\begin{array}{c}\text { Yes } \\
x^{2}(1, \mathrm{~N}=110)= \\
106.04^{*}\end{array}$ & $\begin{array}{c}\text { Yes } \\
x^{2}(1, \mathrm{~N}= \\
109)=22.03^{*}\end{array}$ & & 1 \\
\hline SID & $\begin{array}{l}\text { Textual } \\
\text { Information }\end{array}$ & Speed restrictions & $\begin{array}{c}\text { Yes } \\
x^{2}(1, \mathrm{~N}=110)= \\
106.04^{*}\end{array}$ & $\begin{array}{c}\text { Yes } \\
x^{2}(1, \mathrm{~N}= \\
109)=16.96^{*}\end{array}$ & & 1 \\
\hline SID & Communications & $\begin{array}{l}\text { Departure Control } \\
\text { Frequency }\end{array}$ & $\begin{array}{c}\text { Yes } \\
x^{2}(1, \mathrm{~N}=110)= \\
110.00^{*}\end{array}$ & $\begin{array}{c}\text { Yes } \\
x^{2}(1, \mathrm{~N}= \\
110)=13.13^{*}\end{array}$ & & 1 \\
\hline SID & Communications & $\begin{array}{l}\text { Communications } \\
\text { Boundaries }\end{array}$ & $\begin{array}{c}\text { Yes } \\
x^{2}(1, \mathrm{~N}=110)= \\
94.58^{*}\end{array}$ & $\begin{array}{c}\text { No } \\
x^{2}(1, \mathrm{~N}= \\
106)=25.51^{*}\end{array}$ & $\begin{array}{c}\text { Yes } \\
x^{2}(1, \mathrm{~N}=79) \\
=7.91^{*}\end{array}$ & 2 \\
\hline
\end{tabular}




\begin{tabular}{|l|l|l|c|c|l|l|}
\hline $\begin{array}{l}\text { Chart } \\
\text { Type }\end{array}$ & $\begin{array}{l}\text { Information } \\
\text { Category }\end{array}$ & Information Element & $\begin{array}{l}\text { Q1: Should it be } \\
\text { displayed to } \\
\text { execute } \\
\text { procedure? }\end{array}$ & $\begin{array}{l}\text { Q2: Displayed } \\
\text { at all times? }\end{array}$ & $\begin{array}{l}\text { Q3: Displayed } \\
\text { Initially? }\end{array}$ & $\begin{array}{l}\text { Importance } \\
\text { Level }\end{array}$ \\
\hline SID & Communications & Lost Comm Procedure & $\begin{array}{c}\text { Yes } \\
x^{2}(1, \mathrm{~N}=110)= \\
110.00^{*}\end{array}$ & $\begin{array}{c}\text { No } \\
x^{2}(1, \mathrm{~N}= \\
110)=26.51^{*}\end{array}$ & $\begin{array}{c}\text { No Preference } \\
x^{2}(1, \mathrm{~N}=82) \\
=0.44\end{array}$ & 2 \\
\hline SID & Communications & $\begin{array}{l}\text { Lost Comm Procedure } \\
\text { Outline Lines }\end{array}$ & $\begin{array}{c}\text { Yes } \\
x^{2}(1, \mathrm{~N}=110)= \\
106.04^{*}\end{array}$ & $\begin{array}{c}x^{2}(1, \mathrm{~N}= \\
109)=38.76^{*}\end{array}$ & $\begin{array}{c}\text { No Preference } \\
x^{2}(1, \mathrm{~N}=87) \\
=0.29\end{array}$ & 2 \\
\hline SID & Communications & $\begin{array}{l}\text { Transponder Setting } \\
\text { where appropriate }\end{array}$ & $\begin{array}{c}\text { Yes } \\
x^{2}(1, \mathrm{~N}=108)= \\
88.93^{*}\end{array}$ & $\begin{array}{c}x^{2}(1, \mathrm{~N}= \\
103)=23.31^{*}\end{array}$ & $\begin{array}{c}\text { Yes } \\
x^{2}(1, \mathrm{~N}=76) \\
=23.21^{*}\end{array}$ & 2 \\
\hline
\end{tabular}

